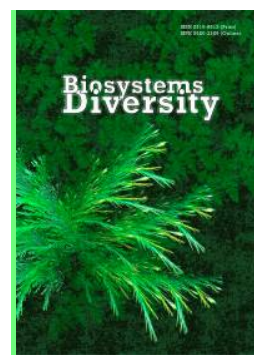

\title{
Biodiversity of the Regional Landscape Park Samara Plavni within the first large reservoir in Europe
}

\author{
B. A. Baranovski*, I. A. Ivanko*, V. J. Gasso*, O. L. Ponomarenko*, D. V. Dubyna**, \\ N. O. Roshchyna***, L. O. Karmyzova*, J. L. Poleva***, V. V. Nikolaieva* \\ * Oles Honchar Dnipro National University, Dnipro, Ukraine \\ **M. G. Kholodny Institute of Botany, National Academy of Sciences of Ukraine, Kyiv, Ukraine \\ ***Dnipro State Agrarian and Economic University, Dnipro, Ukraine
}

Article info

Received 09.04.2021

Received in revised form 12.05 .2021

Accepted 14.05.2021

Oles Honchar Dnipro

National Universit

Gagarin av., 72,

Dnipro, 49010, Ukraine.

Tel.: + 38-095-779-99-94.

E-mail:

boris.baranovki@ukr.net

M. G. Kholodny Institute

of Botany, National Academy

of Sciences of Ukraine,

Tereshchenkivska st., 2 ,

Kyiv, 01004, Ukraine.

Tel.: +38-067-851-37-12.

E-mail:ddub@ukr.net

Dnipro State Agrarian

and Economic University,

Serhii Efremov st., 25 ,

Dnipro, 49600,Ukraine.

Tel.: + 38-097-774-43-27.

E-mail:

botanik.roshchyna@gmail.com
Baranovski, B. A., Ivanko, I. A., Gasso, V. J., Ponomarenko, O. L., Dubyna, D. V., Roshchyna, N. O., Karmyzova, L. O., Poleva, J. L., \& Nikolaieva, V. V. (2021). Biodiversity of the Regional Landscape Park Samara Plavni within the first large reservoir in Europe. Biosystems Diversity, 29(2), 160-179. doi:10.15421/012121

The creation of reservoirs in river valleys for the accumulation of fresh water has been and remains an important issue around the world. This process has both positive and negative consequences for people and nature. Significant changes in the regime of rivers and their valleys, flooding of meadows and forests, flooding of soils and changes in the composition of flora and fauna of adjacent territories are taking place. In this article, we consider the restoration of the biodiversity of a site of disturbed lands after the creation of one of the first large reservoirs in Europe - the Dnieper (Zaporozhe) on the River Dnieper, which has existed since 1933. The territory of the Samara floodplains was formed on the floodplain of the mouth of the Samara River, as a result of which the territories of various forest, meadow and bog biotopes were flooded. For almost 90 years, new biotopes have been developing, and populations of plant and animal species, especially waterfowl, have been renewed and enriched. In the conditions of climate change and anthropogenic pressure, the existence of this territory has fallen into question. To control the conservation of biodiversity, it is necessary to apply various management methods, one of which is creation of nature reserves. The article presents the results of a complex of scientific studies that were carried out during the zoning of the regional park Samara Plavni to improve the management of the protection of water areas and river banks. We have investigated: hydrological features, species diversity of the flora and coenotic diversity of the vegetation, fauna of aquatic invertebrates, and terrestrial vertebrates. Zoning of the Regional Landscape Park was based on the composition of stable components of ecosystems. When applying the protected regime in different zones, conditions will be created for the preservation of habitats of species, including those protected in Europe: higher plants (Senecio borysthenicus (DC.) Andrz. ex Czern., Tragopogon borystenicus Artemcz.), reptiles (Emys orbicularis (Linnaeus, 1758), Vipera renardi (Christoph, 1861)), birds (Aythya ferina (Linnaeus, 1758), Vanellus vanellus (Linnaeus, 1758), Haematopus ostralegus Linnaeus, 1758, Numenius arquata (Linnaeus, 1758), Alcedo atthis (Linnaeus, 1758), Lanius excubitor (Linnaeus, 1758)), mammals (Lutra lutra (Linnaeus, 1758)). Such changes would increase the implementation of the reproductive potential of all species without exception in the studied ecosystems.

Keywords: biodiversity conservation; protected areas; reservoirs of Europe; flora; fauna; zoning of nature reserves.

\section{Introduction}

In Europe, since the late 19th century, legislation has been used to protect species and habitats. Since then, the nature conservation movement has been actively developing and the expansion of protected areas has taken place. After all, the creation nature reserves is an effective tool for the natural restoration of species (Musakwa et al., 2020; Thieme et al., 2020). Conservation and monitoring of biodiversity in protected areas, the importance of zoning of such areas are subjects of debate around the world (Becken et al., 2014; Hülber et al., 2017). In addition, reducing the rate of biodiversity loss has been the major objective of the Convention on Biological Diversity over the last 20 years (Perrings et al., 2010).

Human intervention in natural processes leads to unpredictable consequences. It is especially difficult to calculate all the consequences of such global changes as the flooding of a reservoir. The creation of reservoirs causes significant changes in the hydrological regime of rivers and their valleys: flooding of meadows, forests, flooding of soils and changes in the vegetation of territories (Chakraborty, 2021). Now it is important to determine how to reach a compromise between human water demand and the protection of river ecosystems (Kang et al., 2019). In this paper, we analyze the consequences and results of the restoration of disturbed lands after the creation of one of the first reservoirs in Europe. One of results of the restoration was the creation of a Regional Landscape Park in these lands. A complex of scientific research of the territory has been carried out. It included the study of the hydrological features of the territory, identification of species diversity of flora, the diversity of vegetation cover, fauna of aquatic invertebrates (zoobenthos), and terrestrial vertebrates. Zoning of the Regional Landscape Park was conducted according to the composition of stable components of the ecosystem: flora of vascular plants, zoobenthos, batracofauna, herpetofauna). The examined water area was the Samara Bay, located at the confluence of the Samara River and the Dnieper River, where near-water flooded territories developed as a result of the flooding of the estuarine part of the floodplain of the Samara River during the creation of the first large reservoir in Europe - the Dnieper (Zaporozhye). The floodplain complex of the Samara River, like the floodplain landscapes of Europe in general (Schindler et al., 2016), is characterized by a high species diversity (Brygadyrenko, 2015, 2016; Didur et al., 2019). The Zaporozhye Reservoir was created in 19311934. The flooding of the Samara River floodplain led to the development of floodplains in the area of about 5 thousand hectares. Gradually, conditions were created here for the restoration of old biotypes and development of new biotopes. Until now, in the previously flooded areas of the floodplain, there has been a restoration of the species composition and an increase in biodiversity. 


\section{Materials and methods}

The study area belongs to the regional Samara reach of the reservoir (Baranovsky, 2000), where the river and the lake conditions have combined. This has provided an increase in biodiversity. Within the framework of the Samara Plavni Regional Landscape Park, 2 hydrological sections are separated for the zoning of the Zaporozhye (Dnieper) Reservoir (Baranovsky, 2000). On the upper Novomoskovsky section (above the Novoselovsky bridge), the Samara River bed with its additional reservoirs is flooded. A floodplain-flooded complex was formed here, which united the waters of the branches and tributaries of the Samara River, swamps and meadows, floodplain forests. The lower Novoselovsky area (below the Novoselovsky bridge) is a completely submerged flooded complex.

By geographic regionalization, the basin of the Samara River is located in the territory of the South-West of the East European Plain, in the steppe zone, in the subzone of the Northern Steppe, Orel-Samara and Zaporozhye-Gulyaypol regions of the Dnieper lowland of the Left-BankDnieper north-steppe province. The water area of the Samara Plavni Regional Landscape Park has the length of $9.7 \mathrm{~km}$ and is located in the section 13.6 to $23.3 \mathrm{~km}$ from the former mouth of the Samara River. The Samara River, a left tributary of the Dnieper River, flows through the territory of Donetsk, Kharkov and Dnepropetrovsk regions. The elevation of the source is $186.0 \mathrm{~m}$ above sea level. The mouth of the river is located within the city of Dnipro (Ust-Samara bridge), at $51.2 \mathrm{~m}$ elevation. The coordinates of the mouth are $48^{\circ} 27^{\prime} 21^{\prime \prime} \mathrm{N}$ and $35^{\circ} 06^{\prime} 58^{\prime \prime} \mathrm{E}$. The length of the river is $324.0 \mathrm{~km}$, and the basin area is $22,600 \mathrm{~km}^{2}$. According to the hydrobotanical zoning of the Zaporozhye (Dneprovsky) Reservoir (Baranovsky, 2000), the water area and territory of the regional landscape park belong to the Novomoskovsky and Novoselovsky sections of the Samara reach. The Novomoskovsky area is an area where shallow waters alternate with wooded islands (mainly groves).

The territory of the Samara Plavni Regional Landscape Park is located in the backwater zone of the Zaporozhye (Dnieper) Reservoir. At a certain period, the water levels in the water area of the park corresponded to the water levels in the reservoir. Due to daily fluctuations in the water level in the reservoir, variable water exchange currents and change in the direction of currents in the Samara Bay are observed several times a day. In this regard, in most rivers and canals, the water regime is stagnant and low-flowing. The Samara River and its tributaries: Samarchuk, Podpolnaya, Peschanka and Solyonaya, flow through the park. The floodplain system includes a large number of branches and ducts. Their total length is $34.7 \mathrm{~km}$. The Samara River is navigable from its mouth to the city of Novomoskovsk, including within the framework of the Regional Landscape Park waterway along the river. The Samara is included in the list of "The most important inland waterways of international importance". The navigation channel is regularly cleared along the waterway to ensure the safety of navigation.

The composition and changes in the diversity of flora and fauna in this territory and water area have been studied by scientists since 1980 (Baranovsky, 2000) with regular expedition trips: three trips per year (May, July, October) and a detailed survey of the territory and the water area in 2020 at 12 plots (three plots for each of the four allocated zones of the park). To study the flora of vascular plants in the water areas and the territory of the reserve, a complex of traditional field and cameral methods was applied. The methodological approach was based on the methods of Bel'gard (1950), on the ecomorphic analysis of forest communities and typological principles of the organization of forests in the steppe zone. When studying the flora of vascular plants, we used the methods of collection, herbarization and identification of species and analysis of the collections of the Herbarium of the Oles Honchar Dnipro National University. The species of vascular plants were identified according to the Flora of the European part of the RSFSR (1974-1989) and the Flora of Eastern Europe (1996-2004) using a Citoval microscope (Carl Zeiss Jena). Species names are given according to modem Ukrainian nomenclature publications (Mosyakin \& Fedoronchuk, 1999).

Bioecomorphic characteristics (bioecological certification) of species were compiled according to Bel'gard (1950) and additions made by his followers and the results of their own research. Biomorphs and ecomorphs are given in tabular form using abbreviated symbols: biomorphs (one-, two-, perennials) heliomorphs (relation to light) hygromorphs (relation to the aquatic environment) tropomorphs (relation to trophicity) cenomorphs (growth in certain phytocenoses). The names biomorph and ecomorph are presented according to the ecomorph system of Bel'gard (1950). In the process of ecomorphic analysis of the flora, species that had been found in different biotopes were given identification of complex ecomorphs (for example, HalPalPr), where the latter is considered the main one - Pr). The analysis of the adventive fraction was carried out according to the literature data (Richardson, 2000; Baranovski et al., 2016; Karmyzova \& Baranovsky, 2020). The vegetation cover was investigated according to the generally accepted methods. The analysis of tree and shrub vegetation was carried out according to the typology of Bel'gard (1950).

To characterize the biodiversity of the water areas and territories of the Samara Plavni Regional Landscape Park, studies of the species composition of flora were carried out in various biotopes and ecotones (border areas between two biotopes) according to the European EUNIS system (Didukh et al., 2011). This classification has a hierarchical structure, which makes it possible to generalize information at different levels, reflects ecosystems from natural to artificial, has a successful coding and other features. According to the main biotopes: aquatic biotopes (C), bog biotopes (D), herbaceous biotopes (E), forest biotopes (G), semi-natural biotopes (I) and artificial biotopes (J) with subordinate categories of hygromorphs and cenomorphs are distributed as follows (Table 1).

Table 1

Correspondence of hygromorphs and cenomorphs to the main biotopes of the Samara Plavni Regional Landscape Park

\begin{tabular}{lcc}
\hline \multirow{2}{*}{ Biotopes (according to the EUNIS system) } & \multicolumn{2}{c}{ Main Ecomorphs } \\
\cline { 2 - 4 } C1 Shallow stagnant water bodies & $\mathrm{Pl}, \mathrm{Hel}, \mathrm{Hy}$ & $\mathrm{Aq}, \mathrm{Pal}$ \\
C2 Shallow waters of flowing water bodies & $\mathrm{Hy}, \mathrm{Hel}, \mathrm{Pl}$ & $\mathrm{Aq}$ \\
C3.2 The banks of reservoirs covered with high helophytes & $\mathrm{Hel}$ & $\mathrm{Pal}, \mathrm{Pr}$ \\
D Wetlands biotopes & $\mathrm{Hel}, \mathrm{Hg}$ & $\mathrm{Pal}, \mathrm{Aq}$ \\
E1.2G Sandy steppes & $\mathrm{X}, \mathrm{Ms}$ & $\mathrm{Ps}, \mathrm{St}$ \\
E2 Mesotrophic meadows & $\mathrm{Ms}$ & $\mathrm{Pr}$ \\
E3 Hygrophytic meadows & $\mathrm{Hg}$ & $\mathrm{Pr}, \mathrm{Pal}$ \\
G1 Deciduous forests & $\mathrm{Ms}, \mathrm{X}$ & $\mathrm{Sil}, \mathrm{Ru}$ \\
G3 Coniferous forests & $\mathrm{X}, \mathrm{Ms}$ & $\mathrm{Sil}, \mathrm{Ru}$ \\
F9.35 River banks' thickets of invasive shrubs & $\mathrm{Hg}$ & $\mathrm{Ru}, \mathrm{Sil}$ \\
I1.22 Garden plots & $\mathrm{Ms}$ & $\mathrm{Cu}, \mathrm{Sil}, \mathrm{Ru}$ \\
J2 Scattered building & $\mathrm{Ms}$ & $\mathrm{Ru}, \mathrm{Cu}, \mathrm{Sil}$ \\
\hline
\end{tabular}

For qualitative evaluation, zoobenthos was sampled using a net, mainly in the thickets of higher aquatic vegetation. An Ekman-Burge model bottom bucket with the area of $1 / 40 \mathrm{~m}^{2}$ was used for quantitative sampling. Samples were taken twice, the content of the bottom bucket was poured into a large container, from which the organisms were successively washed from the soil, transferred to laboratory glassware, and immediately fixated in $40 \%$ formalin. Further qualitative and quantitative analyses of the samples were carried out in the laboratory. The species identification was carried out according to the identification keys. The species identification and distribution of amphibians and reptiles was carried out using the standard methods of route counting and traps (McDiarmid et al., 2012; Graeter et al., 2013; Ali et al., 2018). The species composition of birds was determined visually using optical instruments, according to singing, and existing nests (Tomialojc, 1976, 1980; Kuchynska, 2005). The study of mammals was carried out by traces and visually, murine rodents and small insectivores - using traps (Hoffmann et al., 2010; Torre et al., 2018; Green et al., 2020). According to the zoning of the Samara Plavni Regional Landscape Park, the following parts are distinguished: a reserved zone, a recreational zone, a zone of regulated recreation and an economic zone. The reserved zone has two subzones: the upper one - in the Novomoskovsk area; the lower one is in the Novoselovsky site), the recreational zone occupies the main part of the regional landscape park, the regulated recreation zone is located in the Novomoskovsky site, the economic zone is also located in two sites (Fig. 1).

\section{Results}

Floristic diversity. The list of flora of vascular plants of the Samara Plavni Regional Landscape Park contains 626 species belonging to 90 
families. Ecomorphic analysis shows that hemicryptophytes predominate among climamorphs; perennials predominate among biomorphs; scioheliophytes predominate among heliomorphs; mesotrophs predominate among trophomorphs; mesophytes and hygrophytes predominate among hygromorphs. Among the cenomorphs, most species are represented by meadow (233) and forest (206) species. The ruderal flora includes
251 species, including 140 adventive and 5 invasive species. The rare fraction comprises 70 species. Two of them are included in the European Red List, and 15 species are included in the Red Book of Ukraine (Table 2). All of them are included in the list of rare species of vascular plants in Dnepropetrovsk region. Most of them grow in protected and recreational areas.

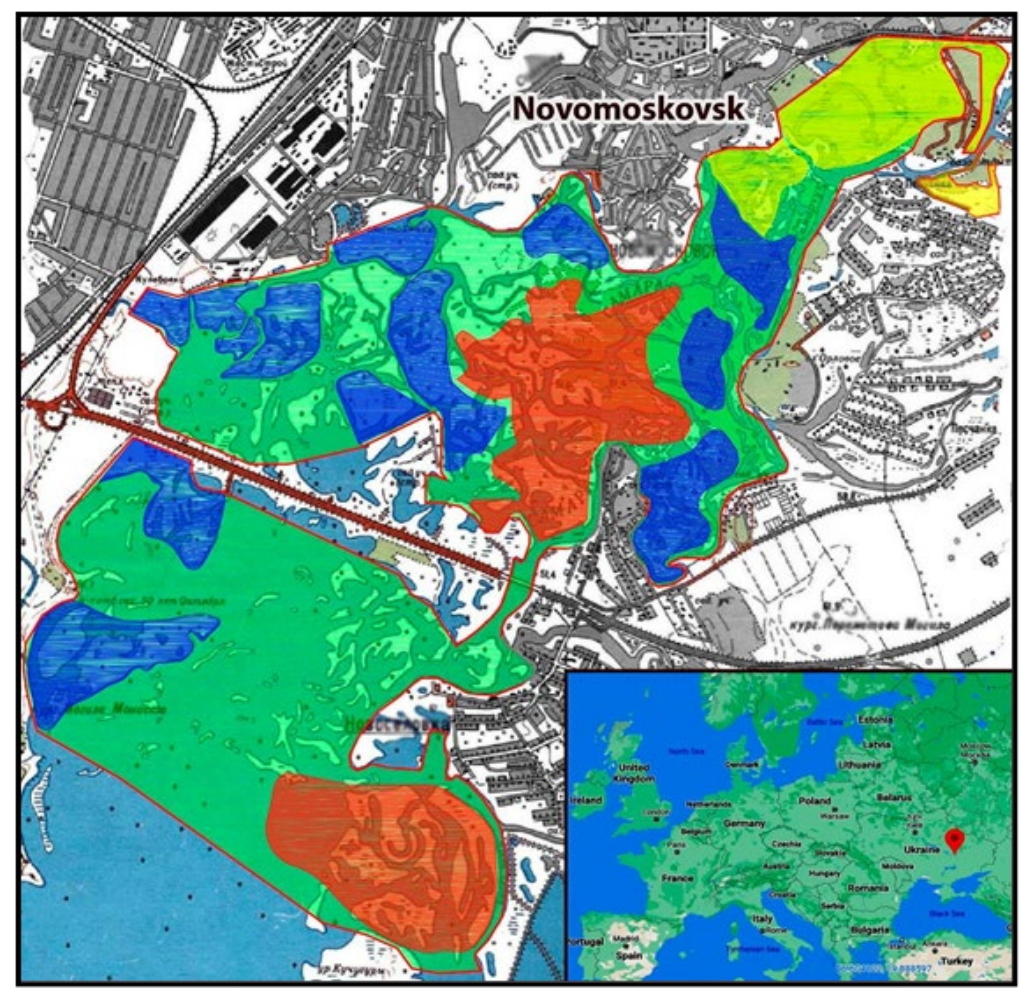

Fig. 1. Zoning of the Samara Plavni Regional Landscape Park: the red line - the boundaries of the Samara Plavni Regional Landscape Park, red - the reserved zone, green - the zone of regulated recreation, yellow - the zone of stationary recreation, blue - the economic zone

Table 2

List of flora of vascular plants of the Samara Plavni Regional Landscape Park by zoning

\begin{tabular}{|c|c|c|c|c|c|c|c|c|c|c|c|c|}
\hline Families & Species & $\begin{array}{l}\text { Clima- } \\
\text { morphs }\end{array}$ & $\begin{array}{c}\text { Bio- } \\
\text { morphs }\end{array}$ & $\begin{array}{l}\text { Helio- } \\
\text { morphs }\end{array}$ & $\begin{array}{l}\text { Tropho- } \\
\text { morphs }\end{array}$ & $\begin{array}{l}\text { Hygro- } \\
\text { morphs }\end{array}$ & $\begin{array}{l}\text { Ceno- } \\
\text { morphs }\end{array}$ & $\begin{array}{l}\text { Protec- } \\
\text { ted zone }\end{array}$ & $\begin{array}{c}\text { Recre- } \\
\text { ational } \\
\text { zone } \\
\end{array}$ & $\begin{array}{c}\text { Stationary } \\
\text { recreation } \\
\text { zone }\end{array}$ & $\begin{array}{l}\text { Econo- } \\
\text { mic zone }\end{array}$ & $\begin{array}{c}\text { Rare, ad- } \\
\text { ventive, } \\
\text { types }\end{array}$ \\
\hline Equisetaceae & Equisetum arvense $\mathrm{L}$. & G & Per & $\mathrm{ScHe}$ & $\mathrm{MsTr}$ & $\mathrm{HgMs}$ & RuSilPr & + & + & + & + & - \\
\hline Equisetaceae & E. fluviatile L. & $\mathrm{HKr}$ & Per & $\mathrm{ScHe}$ & $\mathrm{MsTr}$ & $\mathrm{HelHg}$ & AqPal & + & - & - & - & RLD3 \\
\hline Equisetaceae & E. hyemale L. & $\mathrm{Ch}$ & $\mathrm{sFr}$ & $\mathrm{ScHe}$ & $\operatorname{MsTr}$ & $\mathrm{HgMs}$ & PrSil & + & - & - & - & RLD 3 \\
\hline Dryopteriaceae & Dryopteris carthusiana H. P. Fuchs. & $\mathrm{HKr}$ & Per & $\mathrm{ScHe}$ & $\operatorname{MsTr}$ & Ms & Sil & + & - & - & - & RLD 3 \\
\hline Dryopteriaceae & D. filix-mas (L.) Schott & $\mathrm{HKr}$ & Per & $\mathrm{HeSc}$ & OgMsTr & Ms & Sil & + & - & - & - & RLD3 \\
\hline Salviniaceae & Salvinia natans (L.) All. & $\mathrm{T}$ & Ann & $\mathrm{ScHe}$ & MsTr & Pler & $\mathrm{Aq}$ & + & + & + & + & $\begin{array}{c}\text { RBUU, } \\
\text { RLD3 }\end{array}$ \\
\hline Thelypteridaceae & Thelypteris palustris Schott & $\mathrm{G}$ & Per & $\mathrm{HeSc}$ & MsTr & $\mathrm{Hg}$ & SilPal & + & + & - & - & RLD2 \\
\hline Pinaceae & Pinus pallasiana D. Don & $\mathrm{Ph}$ & Arb & $\mathrm{ScHe}$ & OgMsTr & $\mathrm{XMs}$ & Sil & - & + & + & + & - \\
\hline Pinaceae & P. sylvestris L. & $\mathrm{Ph}$ & Arb & $\mathrm{ScHe}$ & OgMsTr & $\mathrm{X}-\mathrm{Hg}$ & Sil & + & + & + & - & - \\
\hline Alismataceae & Alisma gramineum Lej. & $\mathrm{HKr}$ & Per & $\mathrm{He}$ & MsTr & $\mathrm{HelHg}$ & PalAq & + & + & - & - & - \\
\hline Alismataceae & A. lanceolatum With. & $\mathrm{HKr}$ & Per & $\mathrm{He}$ & $\operatorname{MgTr}$ & $\mathrm{HelHg}$ & PalAq & + & + & - & - & - \\
\hline Alismataceae & A. plantago-aquatica $\mathrm{L}$. & $\mathrm{HKr}$ & Per & $\mathrm{ScHe}$ & $\mathrm{MsTr}$ & $\mathrm{HgHel}$ & PalAq & + & + & + & + & - \\
\hline Alismataceae & Sagittaria sagittifolia $\mathrm{L}$. & $\mathrm{HKr}$ & Per & $\mathrm{ScHe}$ & $\mathrm{MsTr}$ & HgHy & PalAq & + & - & - & - & - \\
\hline Alliaceae & Allium angulosum $\mathrm{L}$. & $\mathrm{G}$ & Per & $\mathrm{ScHe}$ & $\mathrm{MsTr}$ & $\mathrm{HgMs}$ & $\operatorname{Pr}$ & + & - & - & - & - \\
\hline Alliaceae & A. flavescens Bess. & G & Per & $\mathrm{He}$ & $\operatorname{MsTr}$ & MsX & PsPtSt & + & + & + & - & - \\
\hline Alliaceae & A. paczoskianum Tuzs. & $\mathrm{G}$ & Per & $\mathrm{He}$ & $\mathrm{MsTr}$ & $\mathrm{MsX}$ & PsSt & - & + & + & - & - \\
\hline Alliaceae & A. waldschteinii $\mathrm{G}$. Don fil. & G & Per & $\mathrm{He}$ & $\mathrm{MsTr}$ & XMs & StPr & + & + & + & + & - \\
\hline Araceae & Acorus calamus L. & $\mathrm{HKr}$ & Per & $\mathrm{He}$ & MsTr & $\mathrm{HgHel}$ & PalAq & + & + & - & - & RLD3,Adv \\
\hline Asparagaceae & Asparagus officinalis $\mathrm{L}$. & $\mathrm{HKr}$ & Per & $\mathrm{ScHe}$ & $\operatorname{MgTr}$ & $\mathrm{XMs}$ & $\mathrm{PrSt}$ & + & + & + & - & - \\
\hline Butomaceae & Butomus umbellatus L. & $\mathrm{HKr}$ & Per & $\mathrm{He}$ & $\mathrm{MsTr}$ & $\mathrm{HgHel}$ & PalAq & + & + & + & - & - \\
\hline Convallariaceae & Convallaria majalis $\mathrm{L}$. & $\mathrm{G}$ & Per & $\mathrm{HeSc}$ & $\mathrm{MsTr}$ & $\mathrm{HgMs}$ & Sil & + & + & - & - & RLD 3 \\
\hline Convallariaceae & Polygonatum multiflorum (L.) All. & G & Per & $\mathrm{Sc}$ & $\operatorname{MgTr}$ & Ms & Sil & + & + & + & - & - \\
\hline Convallariaceae & P. odoratum (Mill.) Druce & $\mathrm{G}$ & Per & $\mathrm{ScHe}$ & OgTr & Ms & Sil & + & - & - & - & RLD 3 \\
\hline Cyperaceae & Bolboschoenus maritimus (L.) Palla & $\mathrm{HKr}$ & Per & $\mathrm{He}$ & AlkMgTr & $\mathrm{HelHg}$ & AqPal & + & + & + & + & - \\
\hline Cyperaceae & Carexacuta L. & $\mathrm{HKr}$ & Per & $\mathrm{He}$ & MsTr & $\mathrm{HelHg}$ & AqPal & + & + & + & + & - \\
\hline Cyperaceae & C. acutiformis Ehrh. & $\mathrm{HKr}$ & Per & $\mathrm{ScHe}$ & $\mathrm{MsTr}$ & $\mathrm{Hg}$ & SilPal & + & - & - & - & - \\
\hline Cyperaceae & C. bueckii Wimm. & $\mathrm{HKr}$ & Per & $\mathrm{He}$ & AlkMgTr & $\mathrm{HgMs}$ & PalPr & + & + & - & - & RLD 4 \\
\hline Cyperaceae & C. distans $\mathrm{L}$. & $\mathrm{HKr}$ & Per & $\mathrm{He}$ & AlkMgTr & HgMs & PrHal & - & + & - & - & - \\
\hline Cyperaceae & C. disticha Huds. & $\mathrm{HKr}$ & Per & $\mathrm{He}$ & MsTr & HsMs & PalPr & + & - & - & - & - \\
\hline Cyperaceae & C. ligerica J.Gay. & $\mathrm{HKr}$ & Per & $\mathrm{He}$ & OgTr & Ms & Ps & + & + & + & + & - \\
\hline Cyperaceae & C. melanostachya Bieb. ex Willd. & $\mathrm{HKr}$ & Per & $\mathrm{ScHe}$ & AlkMgTr & Ms & PalPrSil & + & + & + & + & - \\
\hline
\end{tabular}




\begin{tabular}{|c|c|c|c|c|c|c|c|c|c|c|c|c|}
\hline Families & Species & $\begin{array}{l}\text { Clima- } \\
\text { morphs }\end{array}$ & $\begin{array}{l}\text { Bio- } \\
\text { morphs }\end{array}$ & $\begin{array}{l}\text { Helio- } \\
\text { morphs }\end{array}$ & $\begin{array}{l}\text { Tropho- } \\
\text { morphs }\end{array}$ & $\begin{array}{l}\text { Hygro- } \\
\text { morphs }\end{array}$ & $\begin{array}{l}\text { Ceno- } \\
\text { morphs }\end{array}$ & $\begin{array}{l}\text { Protec- } \\
\text { ted zone }\end{array}$ & $\begin{array}{c}\text { Recre- } \\
\text { ational } \\
\text { zone }\end{array}$ & $\begin{array}{c}\text { Stationary } \\
\text { recreation } \\
\text { zone }\end{array}$ & $\begin{array}{l}\text { Econo- } \\
\text { mic zone }\end{array}$ & $\begin{array}{c}\text { Rare, ad- } \\
\text { ventive, } \\
\text { types }\end{array}$ \\
\hline Cyperaceae & C. muricata L. & $\mathrm{HKr}$ & Per & $\mathrm{ScHe}$ & $\operatorname{MgTr}$ & Ms & Sil & + & - & - & - & - \\
\hline Cyperaceae & C. otrubae Podp. & $\mathrm{HKr}$ & Per & $\mathrm{ScHe}$ & $\operatorname{MgTr}$ & $\mathrm{HgMs}$ & HalSilPr & + & + & + & + & - \\
\hline Cyperaceae & C. praecox Schreb. & $\mathrm{HKr}$ & Per & $\mathrm{He}$ & $\operatorname{MgTr}$ & $\mathrm{XMs}$ & StPr & + & + & + & + & - \\
\hline Cyperaceae & C. pseudocyperus L. & $\mathrm{HKr}$ & Per & $\mathrm{HeSc}$ & OgMsTr & $\mathrm{Hg}$ & SilPal & + & - & - & - & - \\
\hline Cyperaceae & C. riparia Curtis & $\mathrm{HKr}$ & Per & $\mathrm{He}$ & MsTr & $\mathrm{Hg}$ & Pal & + & + & + & + & - \\
\hline Cyperaceae & C. spicata Huds. & $\mathrm{HKr}$ & Per & $\mathrm{HeSc}$ & $\operatorname{MgTr}$ & XMs & PrSil & + & - & - & - & - \\
\hline Cyperaceae & C. vesicaria $\mathrm{L}$. & $\mathrm{HKr}$ & Per & $\mathrm{He}$ & MsTr & $\mathrm{MsHg}$ & PrPal & + & + & + & - & - \\
\hline Cyperaceae & C. vulpina $\mathrm{L}$. & $\mathrm{HKr}$ & Per & $\mathrm{He}$ & $\operatorname{MsTr}$ & $\mathrm{MsHg}$ & $\mathrm{PalPr}$ & + & + & + & + & - \\
\hline Cyperaceae & Cyperus fuscus $\mathrm{L}$. & $\mathrm{T}$ & Ann & $\mathrm{He}$ & OgMsTr & $\mathrm{Hg}$ & PsPal & + & + & - & - & - \\
\hline Cyperaceae & C. glomeratus L. & $\mathrm{T}$ & Ann & $\mathrm{He}$ & OgMsTr & $\mathrm{Hg}$ & PalPs & + & - & - & - & RLD 1 \\
\hline Cyperaceae & Eleocharis palustris (L.) Roem. et Schult. & $\mathrm{HKr}$ & Per & $\mathrm{He}$ & $\operatorname{MgTr}$ & $\mathrm{Hg}$ & PrPal & + & + & + & + & - \\
\hline Cyperaceae & Scirpoides holoschoenus (L.) Sojak. & $\mathrm{G}$ & Per & $\mathrm{He}$ & OgTr & $\mathrm{HgMs}$ & PrPs & + & + & + & - & - \\
\hline Cyperaceae & Scirpus lacustris L. & $\mathrm{HKr}$ & Per & $\mathrm{He}$ & MsTr & $\mathrm{HelHg}$ & AqPal & + & + & + & + & - \\
\hline Cyperaceae & S. sylvaticus L. & $\mathrm{HKr}$ & Per & $\mathrm{HeSc}$ & MsTr & $\mathrm{Hg}$ & SilPal & + & - & - & - & - \\
\hline Cyperaceae & S. tabernaemontani C. C. Gmel. & $\mathrm{HKr}$ & Per & $\mathrm{He}$ & AlkMsTr & $\mathrm{Hg}$ & Pal & + & + & - & - & - \\
\hline Hyacintaceae & Hyacinthella leucophaea (C.Koch) Schur & $\mathrm{G}$ & Per & $\mathrm{He}$ & MsTr & $\mathrm{MsX}$ & PtSt & - & + & - & - & RLD3 \\
\hline Hyacintaceae & Muscari neglectum Guss. & G & Per & $\mathrm{He}$ & $\mathrm{MsTr}$ & $\mathrm{XMs}$ & SMnSt & + & + & - & - & RLD 2 \\
\hline Hyacintaceae & $\begin{array}{l}\text { Ornithogalum bouscheanum (Kunth) } \\
\text { Aschers. }\end{array}$ & G & Per & $\mathrm{HeSc}$ & MsTr & Ms & PrSil & + & - & - & - & $\begin{array}{l}\text { RBUB, } \\
\text { RLD3 }\end{array}$ \\
\hline Hyacintaceae & O fischieranum Krasch. & G & Per & $\mathrm{He}$ & AlkMsTr & MsX & HalPrSt & + & + & - & - & - \\
\hline Hyacintaceae & O. fimbriatum Willd. & G & Per & $\mathrm{ScHe}$ & MsTr & Ms & Sil & + & - & - & - & RLD 2 \\
\hline Hyacintaceae & Scilla bifolia $\mathrm{L}$ & G & Per & $\mathrm{ScHe}$ & $\operatorname{MsTr}$ & $\mathrm{XMs}$ & StSil & + & + & - & - & RLD3 \\
\hline Hyacintaceae & S. sibirica Haw. & $\mathrm{G}$ & Per & $\mathrm{HeSc}$ & $\operatorname{MgTr}$ & Ms & Sil & + & - & - & - & RLD3 \\
\hline Hydrocharitaceae & Elodea canadensis Michx. & $\mathrm{Hd}$ & Per & $\mathrm{HeSc}$ & OgMsTr & Hy r & RuAq & + & + & + & - & Adv \\
\hline Hydrocharitaceae & Hydrocharis morsus-ranae L. & $\mathrm{Hd}$ & Per & $\mathrm{ScHe}$ & MsTr & Pler & $\mathrm{Aq}$ & + & + & + & + & - \\
\hline Hydrocharitaceae & Stratiotes aloides L. & $\mathrm{Hd}$ & Per & $\mathrm{ScHe}$ & MsTr & $\mathrm{Plr}$ & $\mathrm{Aq}$ & + & + & - & - & RLD3 \\
\hline Hydrocharitaceae & Vallisneria spiralis L. & $\mathrm{Hd}$ & Per & $\mathrm{HeSc}$ & MsTr & Hyr & $\mathrm{RuAq}$ & + & + & - & - & RLD3,Adv \\
\hline Iridaceae & Crocus reticulatus Stev. ex Adam. & G & Per & $\mathrm{He}$ & AlkMsTr & MsX & PrSt & + & + & - & - & $\begin{array}{l}\text { RBUB, } \\
\text { RLD 2 }\end{array}$ \\
\hline Iridaceae & Gladiolus tenuis Bieb. & G & Per & $\mathrm{ScHe}$ & MsTr & $\mathrm{XMs}$ & $\operatorname{Pr}$ & + & + & - & - & $\begin{array}{l}\text { RBUB, } \\
\text { RLD2 }\end{array}$ \\
\hline Iridaceae & Iris halophila Pall. & $\mathrm{HKr}$ & Per & $\mathrm{He}$ & AlkMsTr & $\mathrm{XMs}$ & HalPr & - & + & - & - & RLD2 \\
\hline Iridaceae & I. pseudacorus L. & $\mathrm{G}$ & Per & $\mathrm{He}$ & MsTr & $\mathrm{Hg}$ & Pal & + & + & + & - & - \\
\hline Juncaceae & Juncus articulatus L. & $\mathrm{HKr}$ & Per & $\mathrm{He}$ & OgMsTr & $\mathrm{MsHg}$ & $\mathrm{PalPr}$ & + & + & - & - & - \\
\hline Juncaceae & J. atratus Krook. & $\mathrm{HKr}$ & Per & $\mathrm{He}$ & $\operatorname{Mg} \operatorname{Tr}$ & $\mathrm{HgMs}$ & $\operatorname{Pr}$ & + & + & - & - & - \\
\hline Juncaceae & J. bufonius L. & $\mathrm{T}$ & Ann & $\mathrm{ScHe}$ & OgMsTr & $\mathrm{MsHg}$ & PsPr & + & + & - & - & - \\
\hline Juncaceae & J. compressus Jacq. & $\mathrm{HKr}$ & Per & $\mathrm{He}$ & $\operatorname{MgTr}$ & $\mathrm{MsHg}$ & PalPr & + & + & + & - & - \\
\hline Juncaceae & J. effusus L. & $\mathrm{Hel}$ & Per & $\mathrm{He}$ & $\mathrm{MsTr}$ & $\mathrm{Hg}$ & Pal & + & + & + & - & - \\
\hline Juncaceae & J. gerardii Loisel. & $\mathrm{HKr}$ & Per & $\mathrm{He}$ & AlkMsTr & $\mathrm{HgMs}$ & HalPr & + & + & + & + & - \\
\hline Juncaceae & J. inflexus $\mathrm{L}$. & $\mathrm{HKr}$ & Per & $\mathrm{He}$ & MsTr & $\mathrm{MsHg}$ & $\operatorname{Pr}$ & + & + & - & - & - \\
\hline Juncaginaceae & Triglochin maritimum $\mathrm{L}$. & $\mathrm{HKr}$ & Per & $\mathrm{He}$ & AlkTr & $\mathrm{MsHg}$ & PalHalPr & + & + & + & + & - \\
\hline Juncaginaceae & T. palustre $\mathrm{L}$. & $\mathrm{HKr}$ & Per & $\mathrm{He}$ & $\operatorname{MgTr}$ & $\mathrm{MsHg}$ & HalPalPr & + & + & - & - & - \\
\hline Lemnaceae & Lemna gibba L. & Hel & Per & $\mathrm{ScHe}$ & MsTr & Pler & $\mathrm{Aq}$ & + & + & - & - & - \\
\hline Lemnaceae & L. minor $\mathrm{L}$. & Hel & Per & $\mathrm{ScHe}$ & $\mathrm{MsTr}$ & Pler & $\mathrm{Aq}$ & + & + & + & + & - \\
\hline Lemnaceae & L. trisulca $\mathrm{L}$. & Hel & Per & $\mathrm{HeSc}$ & $\mathrm{MsTr}$ & Hy er & $\mathrm{Aq}$ & + & + & + & + & - \\
\hline Lemnaceae & Spirodela polirrhyza (L.) Schleid. & Hel & Per & $\mathrm{He}$ & MsTr & Pler & $\mathrm{Aq}$ & + & + & - & - & - \\
\hline Lemnaceae & Wolffia arrhiza (L.) Horkel ex Wimm. & Hel & Per & $\mathrm{He}$ & MsTr & Pler & $\mathrm{Aq}$ & + & + & + & - & RLD3 \\
\hline Liliaceae & $\begin{array}{l}\text { Fritillaria meleagroides Patrin ex Schult. et } \\
\text { Schult. }\end{array}$ & G & Per & $\mathrm{He}$ & AlkMgTr & Ms & $\operatorname{Pr}$ & + & + & - & - & $\begin{array}{l}\text { RBUB, } \\
\text { RLD 2 }\end{array}$ \\
\hline Liliaceae & Gagea erubscens (Bess.) Schult. et Schult. & $\mathrm{G}$ & Per & $\mathrm{ScHe}$ & $\operatorname{MgTr}$ & Ms & RuSil & + & + & + & + & - \\
\hline Liliaceae & G. lutea (L.) Ker-Gawl. & G & Per & $\mathrm{HeSc}$ & $\mathrm{MsTr}$ & Ms & Sil & + & + & - & - & RLD 3 \\
\hline Liliaceae & G. pusilla (F.W.Schmidt) Schult. et Schult. & G & Per & $\mathrm{He}$ & $\operatorname{MgTr}$ & $\mathrm{XMs}$ & RuSt & & + & + & + & - \\
\hline Liliaceae & Tulipa quercetorum Klokov et Zoz & $\mathrm{G}$ & Per & $\mathrm{HeSc}$ & $\operatorname{MgTr}$ & Ms & StSil & + & + & + & - & $\begin{array}{l}\text { RBUB, } \\
\text { RLD3 }\end{array}$ \\
\hline Najadaceae & Caulinia minor (All.) Coss. et Germ. & $\mathrm{T}$ & Ann & $\mathrm{HeSc}$ & MsTr & Hy r & $\mathrm{Aq}$ & + & + & - & - & RLD 2 \\
\hline Najadaceae & Najas marina $\mathrm{L}$. & $\mathrm{T}$ & Ann & $\mathrm{HeSc}$ & MsTr & Hy r & $\mathrm{Aq}$ & + & + & + & + & - \\
\hline Orchidaceae & Dactylorhisa incarnata L. & G & Per & $\mathrm{He}$ & $\operatorname{MgTr}$ & $\mathrm{MsHg}$ & PrPal & + & + & - & - & $\begin{array}{l}\text { RBUE, } \\
\text { RLD 1 }\end{array}$ \\
\hline Orchidaceae & Epipactis palustris (L.) Crantz & G & Per & $\mathrm{ScHe}$ & OgMsTr & $\mathrm{MsHg}$ & PalPr & + & + & - & - & $\begin{array}{l}\text { RBUB, } \\
\text { RLD 1 }\end{array}$ \\
\hline Orchidaceae & Orchis coriophora L. & G & Per & $\mathrm{He}$ & $\mathrm{MsTr}$ & $\mathrm{HgMs}$ & PalPr & + & + & - & - & $\begin{array}{l}\text { RBUE, } \\
\text { RLD 0 }\end{array}$ \\
\hline Orchidaceae & O. palustris Jacq. & G & Per & $\mathrm{He}$ & AlkMsTr & $\mathrm{MsHg}$ & PrPal & + & + & - & - & $\begin{array}{l}\text { RBUP, } \\
\text { RLD } 2\end{array}$ \\
\hline Orchidaceae & O. militaris L. & G & Per & $\mathrm{HeSc}$ & $\operatorname{Ms} \operatorname{Tr}$ & Ms & SilPr & + & + & - & - & $\begin{array}{l}\text { RBUB, } \\
\text { RLD2 }\end{array}$ \\
\hline Poaceae & Aegilops cylindrica Host & $\mathrm{T}$ & Ann & $\mathrm{He}$ & OgMsTr & MsX & PsPtRu & - & - & - & + & - \\
\hline Poaceae & Agropyron pectinatum (Bieb.) Beaux & $\mathrm{HKr}$ & Per & $\mathrm{He}$ & MsTr & $\mathrm{X}$ & St & - & + & + & + & - \\
\hline Poaceae & Agrostis gigantea Roth. & $\mathrm{HKr}$ & Per & $\mathrm{ScHe}$ & $\mathrm{MsTr}$ & Ms & SilPr & + & + & - & - & - \\
\hline Poaceae & A. stolonifera $\mathrm{L}$. & $\mathrm{HKr}$ & Per & $\mathrm{ScHe}$ & OgMsTr & $\mathrm{Hg}$ & PrPal & + & + & + & + & - \\
\hline Poaceae & Alopecurus aequalis Sobol. & $\mathrm{T}$ & Ann & $\mathrm{He}$ & $\operatorname{Og} \operatorname{Tr}$ & $\mathrm{HgHel}$ & PrPal & + & + & - & - & - \\
\hline Poaceae & A. arundinaceus Poir. & G & Per & $\mathrm{He}$ & AlkMgTr & $\mathrm{HgMs}$ & HalPalPr & + & + & + & - & - \\
\hline Poaceae & A. geniculatus $\mathrm{L}$. & $\mathrm{T}$ & Ann & $\mathrm{He}$ & OgTr & $\mathrm{HgMs}$ & PalPr & + & + & - & - & - \\
\hline Poaceae & A. pratensis $\mathrm{L}$. & $\mathrm{HKr}$ & Per & $\mathrm{He}$ & $\operatorname{MgTr}$ & $\mathrm{HgMs}$ & Pr & + & + & + & + & - \\
\hline Poaceae & Anisantha sterilis (L.) Nevski & $\mathrm{T}$ & Ann & $\mathrm{ScHe}$ & MsTr & MsX & $\mathrm{PrStRu}$ & - & - & + & + & Adv \\
\hline Poaceae & A. tectorum (L.) Nevski & $\mathrm{T}$ & Ann & $\mathrm{ScHe}$ & $\mathrm{OgMgTr}$ & MsX & $\mathrm{PsRu}$ & + & + & + & + & Adv \\
\hline Poaceae & Apera spica-venti (L.) P. Beauv. & $\mathrm{T}$ & Ann & $\mathrm{ScHe}$ & $\operatorname{OgTr}$ & $\mathrm{XMs}$ & RuPs & - & + & + & + & Adv \\
\hline Poaceae & Avena fatua $\mathrm{L}$. & $\mathrm{T}$ & Ann & $\mathrm{He}$ & MsTr & MsX & $\mathrm{Ru}$ & - & - & + & + & Adv \\
\hline Poaceae & Beckmania eruciformis (L.) Host. & $\mathrm{HKr}$ & Per & $\mathrm{ScHe}$ & AlkMsTr & $\mathrm{HgMs}$ & PalPr & + & + & - & - & - \\
\hline Poaceae & Brachypodium syluaticum (Huds.)P. Beauv. & $\mathrm{HKr}$ & Per & $\mathrm{Sc}$ & $\operatorname{MgTr}$ & Ms & Sil & + & - & - & - & - \\
\hline
\end{tabular}




\begin{tabular}{|c|c|c|c|c|c|c|c|c|c|c|c|c|}
\hline Families & Species & $\begin{array}{l}\text { Clima- } \\
\text { morphs }\end{array}$ & $\begin{array}{l}\text { Bio- } \\
\text { morphs }\end{array}$ & $\begin{array}{l}\text { Helio- } \\
\text { morphs }\end{array}$ & $\begin{array}{l}\text { Tropho- } \\
\text { morphs }\end{array}$ & $\begin{array}{l}\text { Hygro- } \\
\text { morphs }\end{array}$ & $\begin{array}{l}\text { Ceno- } \\
\text { morphs }\end{array}$ & $\begin{array}{l}\text { Protec- } \\
\text { ted zone }\end{array}$ & $\begin{array}{c}\text { Recre- } \\
\text { ational } \\
\text { zone }\end{array}$ & $\begin{array}{c}\text { Stationary } \\
\text { recreation } \\
\text { zone }\end{array}$ & $\begin{array}{l}\text { Econo- } \\
\text { mic zone }\end{array}$ & $\begin{array}{c}\text { Rare, ad- } \\
\text { ventive, } \\
\text { types }\end{array}$ \\
\hline Poaceae & Bromopsis inermis (Leys.) Holub & $\mathrm{G}$ & Per & $\mathrm{He}$ & OgMgTr & $\mathrm{XMs}$ & $\mathrm{RuPrSt}$ & + & + & + & + & - \\
\hline Poaceae & B. riparia (Rehm.) Holub & G & Per & $\mathrm{He}$ & $\mathrm{OgTr}$ & MsX & PrSt & + & + & + & + & - \\
\hline Poaceae & Bromus hordeaceus L. & $\mathrm{T}$ & Ann & $\mathrm{ScHe}$ & $\mathrm{Ms} \operatorname{Tr}$ & $\mathrm{XMs}$ & $\mathrm{Ru}$ & - & - & + & + & - \\
\hline Poaceae & B. squarrosus L. & $\mathrm{T}$ & Ann & $\mathrm{ScHe}$ & OgMgTr & MsX & RuPsSt & - & + & + & + & Adv \\
\hline Poaceae & Calamagrostis canescens (Web.) Roth & $\mathrm{HKr}$ & Per & $\mathrm{ScHe}$ & MsTr & $\mathrm{MsHg}$ & SilPrPal & + & + & + & + & - \\
\hline Poaceae & C. epigeios (L.) Roth & $\mathrm{G}$ & Per & $\mathrm{ScHe}$ & OgMsTr & $\mathrm{Ms}$ & PsSilPr & + & + & + & + & - \\
\hline Poaceae & Cenchrus longispinus (Hack.) Fernald & $\mathrm{T}$ & Ann & $\mathrm{He}$ & $\operatorname{Og} \operatorname{Tr}$ & MsX & PsRu & - & - & + & - & Adv \\
\hline Poaceae & Dactylis glomerata $\mathrm{L}$. & $\mathrm{HKr}$ & Per & $\mathrm{ScHe}$ & OgMsTr & Ms & SilPr & + & + & + & + & - \\
\hline Poaceae & Digitaria sanguinalis (L.) Scop. & $\mathrm{T}$ & Ann & $\mathrm{He}$ & OgMsTr & Ms & PsRu & + & + & + & + & Adv \\
\hline Poaceae & Echinochloa crusgalli (L.) P. Beauv. & $\mathrm{T}$ & Ann & $\mathrm{He}$ & OgMgTr & $\mathrm{MsHg}$ & $\mathrm{Ru}$ & + & + & + & + & Adv \\
\hline Poaceae & Elytrigia repens (L.) Nevski & G & Per & $\mathrm{ScHe}$ & MsTr & $\begin{array}{l}\text { MsX- } \\
\text { MsHg }\end{array}$ & $\begin{array}{l}\text { SilStPr } \\
\mathrm{Ru}\end{array}$ & + & + & + & + & - \\
\hline Poaceae & Eragrostis minor Host & $\mathrm{T}$ & Ann & $\mathrm{He}$ & OgMsTr & MsX & PsRu & + & + & + & + & Adv \\
\hline Poaceae & E. pilosa (L.) P. Beauv. & $\mathrm{T}$ & Ann & $\mathrm{He}$ & OgTr & MsX & RuPtPs & + & - & - & - & Adv \\
\hline Poaceae & Festuca beckeri (Hack) Trautv. & $\mathrm{HKr}$ & Per & $\mathrm{He}$ & OgTr & $\mathrm{X}$ & StSilPs & - & + & + & - & - \\
\hline Poaceae & F. gigantea $(\mathrm{L}$.$) Vill.$ & $\mathrm{HKr}$ & Per & $\mathrm{Sc}$ & $\operatorname{MgTr}$ & $\mathrm{HgMs}$ & Sil & + & + & - & - & - \\
\hline Poaceae & F. pratensis Huds. & $\mathrm{HKr}$ & Per & $\mathrm{ScHe}$ & MsTr & $\mathrm{HgMs}$ & $\operatorname{Pr}$ & + & + & - & - & - \\
\hline Poaceae & F. regeliana Pavl. & $\mathrm{HKr}$ & Per & $\mathrm{He}$ & AlkTr & $\mathrm{MsHg}$ & HalPr & + & + & + & + & - \\
\hline Poaceae & F. valesiaca Gaudin. & $\mathrm{HKr}$ & Per & $\mathrm{He}$ & $\operatorname{MgTr}$ & $x^{\circ}$ & St & - & + & + & - & - \\
\hline Poaceae & Glyceria maxima (C. Hartm.) Holub. & $\mathrm{HKr}$ & Per & $\mathrm{He}$ & $\mathrm{Ms} T r$ & $\mathrm{HgHel}$ & PalAq & + & + & - & - & - \\
\hline Poaceae & G. notata (Chevall.) & $\mathrm{HKr}$ & Per & $\mathrm{He}$ & $\mathrm{Ms} T r$ & $\mathrm{Hg}$ & PrPal & + & - & - & - & - \\
\hline Poaceae & Hierochloe odorata (L.) P. Beauv. & $\mathrm{G}$ & Per & $\mathrm{ScHe}$ & OgMsTr & $\mathrm{XMs}$ & SilStPr & + & + & - & - & - \\
\hline Poaceae & Hordeum murinum L. & $\mathrm{T}$ & Ann & $\mathrm{He}$ & MsTr & MsX & RuPsSt & - & - & + & + & Adv \\
\hline Poaceae & Koeleria sabuletorum (Domin) Klokov & $\mathrm{HKr}$ & Per & $\mathrm{He}$ & OgTr & MsX & PsSt & - & + & + & - & - \\
\hline Poaceae & Leersia orizoides (L.) Sw. & $\mathrm{G}$ & Per & $\mathrm{HeSc}$ & OgMsTr & $\mathrm{HelHg}$ & PrPal & + & - & - & - & RLD3 \\
\hline Poaceae & Lolium perenne L. & $\mathrm{HKr}$ & Per & $\mathrm{He}$ & $\operatorname{MgTr}$ & $\mathrm{XMs}$ & $\mathrm{RuPr}$ & + & + & + & + & - \\
\hline Poaceae & Melica altissima L. & $\mathrm{HKr}$ & Per & $\mathrm{ScHe}$ & $\mathrm{MsTr}$ & $\mathrm{XMs}$ & SMr & + & - & - & - & - \\
\hline Poaceae & Molinia caerulea (L.) Moench & $\mathrm{HKr}$ & Per & $\mathrm{ScHe}$ & OgTr & $\mathrm{Hg}$ & SilPrPal & + & - & - & - & RLD 2 \\
\hline Poaceae & Phalaroides arundinacea (L.) Rauschert & $\mathrm{HKr}$ & Per & $\mathrm{ScHe}$ & $\operatorname{MgTr}$ & $\mathrm{MsHg}$ & PrPal & + & + & + & - & - \\
\hline Poaceae & Phleum pratense L. & $\mathrm{HKr}$ & Per & $\mathrm{He}$ & $\operatorname{MgTr}$ & $\mathrm{Ms}$ & $\operatorname{Pr}$ & + & + & - & - & - \\
\hline Poaceae & Phragmites australis (Cav.) Trin. ex Steud. & $\mathrm{HKr}$ & Per & $\mathrm{ScHe}$ & $\operatorname{MsTr}$ & Hel & PalAq & + & + & + & + & - \\
\hline Poaceae & Poa angustifolia $\mathrm{L}$. & $\mathrm{HKr}$ & Per & $\mathrm{ScHe}$ & MsMgTr & MsX & SilPrSt & + & + & + & + & - \\
\hline Poaceae & P. annua $\mathrm{L}$. & $\mathrm{T}$ & Ann & $\mathrm{HeSc}$ & MsTr & Ms & RuSilPr & + & + & + & + & - \\
\hline Poaceae & P. bulbosa $\mathrm{L}$. & $\mathrm{HKr}$ & Per & $\mathrm{He}$ & OgMsTr & MsX & RuSilSt & + & + & + & + & - \\
\hline Poaceae & P. compressa $\mathrm{L}$. & $\mathrm{HKr}$ & Per & $\mathrm{ScHe}$ & OgMsTr & MsX & RuSt & + & + & + & + & - \\
\hline Poaceae & P. nemoralis L. & $\mathrm{HKr}$ & Per & $\mathrm{ScHe}$ & MsTr & $\mathrm{XMs}$ & Sil & + & + & - & - & - \\
\hline Poaceae & P. palustris L. & $\mathrm{HKr}$ & Per & $\mathrm{He}$ & MsTr & $\mathrm{MsHg}$ & PalPr & + & + & + & - & - \\
\hline Poaceae & P. pratensis L. & $\mathrm{G}$ & Per & $\mathrm{He}$ & $\mathrm{MsTr}$ & $\mathrm{Ms}$ & $\operatorname{Pr}$ & + & + & + & + & - \\
\hline Poaceae & P. sylvicola Guss. & $\mathrm{HKr}$ & Per & $\mathrm{HeSc}$ & $\operatorname{MgTr}$ & $\mathrm{HgMs}$ & SilPalPr & + & - & - & - & - \\
\hline Poaceae & P. trivialis L. & $\mathrm{HKr}$ & Per & $\mathrm{He}$ & $\mathrm{MsTr}$ & $\mathrm{HgMs}$ & SilPalPr & + & - & - & - & - \\
\hline Poaceae & Puccinella distans (Jacq.) Parl. & $\mathrm{HKr}$ & Per & $\mathrm{He}$ & AlkMsTr & $\mathrm{XMs}$ & RuHalPr & - & + & - & - & - \\
\hline Poaceae & Sclerochloa dura (L.) P. Beauv. & $\mathrm{T}$ & Ann & $\mathrm{He}$ & MsTr & $\mathrm{XMs}$ & $\mathrm{StRu}$ & - & - & - & + & Adv \\
\hline Poaceae & Secale sylvestre Host & $\mathrm{T}$ & Ann & $\mathrm{He}$ & $\operatorname{OgTr}$ & MsX & StRuPs & - & + & + & + & - \\
\hline Poaceae & Setaria glauca (L.) P. Beauv. & $\mathrm{T}$ & Ann & $\mathrm{He}$ & $\mathrm{MsTr}$ & $\mathrm{XMs}$ & PsRu & - & + & + & + & Adv \\
\hline Poaceae & S. verticillata (L.) H. B. & $\mathrm{T}$ & Ann & $\mathrm{ScHe}$ & $\operatorname{MgTr}$ & Ms & $\mathrm{Ru}$ & - & - & + & + & Adv \\
\hline Poaceae & S. viridis (L.) P. Beauv. & $\mathrm{T}$ & Ann & $\mathrm{He}$ & OgMsTr & $\mathrm{XMs}$ & PsRu & + & + & + & + & Adv \\
\hline Poaceae & Stipa capillata $\mathrm{L}$. & $\mathrm{HKr}$ & Per & $\mathrm{He}$ & MsTr & $X$ & PtSt & - & + & + & + & $\begin{array}{l}\text { RBUU, } \\
\text { RLD 3 }\end{array}$ \\
\hline Potamogetonaceae & Potamogeton berchtoldii Fieb. & $\mathrm{HKr}$ & Per & $\mathrm{HeSc}$ & $\operatorname{MsTr}$ & Hyr & $\mathrm{Aq}$ & + & + & + & + & - \\
\hline Potamogetonaceae & P. compressus $\mathrm{L}$. & $\mathrm{HKr}$ & Per & $\mathrm{HeSc}$ & $\mathrm{MsTr}$ & Hyr & $\mathrm{Aq}$ & + & + & - & - & - \\
\hline Potamogetonaceae & P. crispus $\mathrm{L}$. & $\mathrm{HKr}$ & Per & $\mathrm{HeSc}$ & $\operatorname{MsTr}$ & Hy $r$ & $\mathrm{Aq}$ & + & + & + & + & - \\
\hline Potamogetonaceae & P. gramineus $\mathrm{L}$. & $\mathrm{HKr}$ & Per & $\mathrm{ScHe}$ & MsTr & PIHyr & $\mathrm{Aq}$ & + & + & - & - & RLD 3 \\
\hline Potamogetonaceae & P. lucens L. & $\mathrm{HKr}$ & Per & $\mathrm{HeSc}$ & MsTr & Hyr & $\mathrm{Aq}$ & + & + & - & - & - \\
\hline Potamogetonaceae & P. pectinatus $\mathrm{L}$. & $\mathrm{HKr}$ & Per & $\mathrm{HeSc}$ & $\mathrm{MsTr}$ & Hy r & $\mathrm{Aq}$ & + & + & + & + & - \\
\hline Potamogetonaceae & P. perfoliatus $\mathrm{L}$. & $\mathrm{HKr}$ & Per & $\mathrm{HeSc}$ & MsTr & Hy r & $\mathrm{Aq}$ & + & + & + & + & - \\
\hline Sparganiaceae & Sparganium erectum $\mathrm{L}$. & $\mathrm{HKr}$ & Per & $\mathrm{He}$ & MsTr & $\mathrm{Hel}$ & $\mathrm{PalAq}$ & + & + & - & - & - \\
\hline Sparganiaceae & S. minimum Wallr. & $\mathrm{HKr}$ & Per & $\mathrm{HeSc}$ & $\mathrm{MsTr}$ & Hel & PalAq & + & - & - & - & RLD 4 \\
\hline Typhaceae & Typha angustifolia $\mathrm{L}$. & $\mathrm{HKr}$ & Per & $\mathrm{He}$ & $\operatorname{MsTr}$ & Hel & PalAq & + & + & + & + & - \\
\hline Typhaceae & T.latifolia $\mathrm{L}$. & $\mathrm{HKr}$ & Per & $\mathrm{He}$ & $\operatorname{MgTr}$ & Hel & PalAq & + & + & + & + & - \\
\hline Typhaceae & T. laxmannii Lepech. & $\mathrm{HKr}$ & Per & $\mathrm{He}$ & AlkMsTr & $\mathrm{Hel}$ & PalAq & + & + & + & + & - \\
\hline Zannicheliaceae & Zannichelia palustris $\mathrm{L}$. & $\mathrm{HKr}$ & Per & $\mathrm{HeSc}$ & AlkMsTr & Hyr & $\mathrm{Aq}$ & + & + & - & - & - \\
\hline Aceraceae & Acer campestre L. & $\mathrm{Ph}$ & Arb & $\mathrm{ScHe}$ & MgMsTr & $\mathrm{XMs}$ & SMnSil & + & + & - & - & - \\
\hline Aceraceae & A. negundo L. & $\mathrm{Ph}$ & Arb & $\mathrm{He}$ & $\mathrm{Og}-\mathrm{MgTr}$ & $\begin{array}{l}\text { MsX- } \\
\text { HgMs }\end{array}$ & SilCuRu & + & + & + & + & Adv, Inv \\
\hline Aceraceae & A. platanoides $\mathrm{L}$. & $\mathrm{Ph}$ & Arb & $\mathrm{HeSc}$ & MgMsTr & Ms & Sil & + & + & - & - & - \\
\hline Aceraceae & A. tataricum $\mathrm{L}$. & $\mathrm{Ph}$ & ArbFr & $\mathrm{ScHe}$ & Og-MgTr & $\begin{array}{l}\text { MsX- } \\
\text { HgMs }\end{array}$ & SilSMn & + & + & + & + & - \\
\hline Adoxaceae & Adoxa moschatellina $\mathrm{L}$. & $\mathrm{HKr}$ & Per & $\mathrm{HeSc}$ & MsTr & Ms & Sil & + & - & - & - & RLD 4 \\
\hline Amaranthaceae & Amaranthus albus L. & $\mathrm{T}$ & Ann & $\mathrm{He}$ & MsTr & MsX & $\mathrm{Ru}$ & - & + & + & + & Adv \\
\hline Amaranthaceae & A. blitoides $\mathrm{S}$. Wats. & $\mathrm{T}$ & Ann & $\mathrm{He}$ & MsTr & MsX & $\mathrm{Ru}$ & - & + & + & + & Adv \\
\hline Amaranthaceae & A. retroflexus L. & $\mathrm{T}$ & Ann & $\mathrm{He}$ & $\mathrm{MsTr}$ & MsX & $\mathrm{Ru}$ & + & + & + & + & Adv \\
\hline Anacardiaceae & Cotinus coggygria Scop. & $\mathrm{Ph}$ & $\mathrm{Fr}$ & $\mathrm{ScHe}$ & $\mathrm{Og}-\mathrm{MgTr}$ & MsX & $\mathrm{SMnCu}$ & - & + & + & + & Adv \\
\hline Anacardiaceae & Rhus typhina $\mathrm{L}$. & $\mathrm{Ph}$ & Arb & $\mathrm{He}$ & $\mathrm{MsTr}$ & MsX & $\mathrm{SilCu}$ & - & - & + & + & Adv \\
\hline Apiaceae & Aegopodium podagraria L. & $\mathrm{G}$ & Per & $\mathrm{HeSc}$ & $\operatorname{MgTr}$ & Ms & Sil & + & - & - & - & - \\
\hline Apiaceae & Aethus a cynapium L. & $\mathrm{HKr}$ & Ann & $\mathrm{ScHe}$ & $\mathrm{MsTr}$ & $\mathrm{XMs}$ & $\mathrm{SilRu}$ & + & + & - & - & Adv \\
\hline Apiaceae & Antriscus cerefolium (L.) Hoffm. & $\mathrm{T}$ & Ann & $\mathrm{HeSc}$ & $\mathrm{MsTr}$ & $\mathrm{XMs}$ & SilRu & + & + & - & - & - \\
\hline Apiaceae & A sylvestris (L.) Hoffm. & $\mathrm{HKr}$ & Per & $\mathrm{HeSc}$ & $\operatorname{MsTr}$ & Ms & RuSil & + & + & + & + & - \\
\hline Apiaceae & Chaerophyllum bulbosum $\mathrm{L}$. & $\mathrm{HKr}$ & Bien & $\mathrm{ScHe}$ & OgTr & Ms & PrSil & + & + & - & - & - \\
\hline Apiaceae & C.prescotii DC. & $\mathrm{G}$ & Bien & $\mathrm{ScHe}$ & $\mathrm{MgTr}$ & Ms & $(\mathrm{Pr}) \mathrm{SilRu}$ & + & + & - & - & - \\
\hline Apiaceae & C. temulum $\mathrm{L}$. & $\mathrm{HKr}$ & Bien & $\mathrm{HeSc}$ & MsTr & Ms & RuSil & + & + & + & + & - \\
\hline
\end{tabular}




\begin{tabular}{|c|c|c|c|c|c|c|c|c|c|c|c|c|}
\hline Families & Species & $\begin{array}{l}\text { Clima- } \\
\text { morphs }\end{array}$ & $\begin{array}{c}\text { Bio- } \\
\text { morphs }\end{array}$ & $\begin{array}{l}\text { Helio- } \\
\text { morphs }\end{array}$ & $\begin{array}{l}\text { Tropho- } \\
\text { morphs }\end{array}$ & $\begin{array}{l}\text { Hygro- } \\
\text { morphs }\end{array}$ & $\begin{array}{l}\text { Ceno- } \\
\text { morphs }\end{array}$ & $\begin{array}{l}\text { Protec- } \\
\text { ted zone }\end{array}$ & $\begin{array}{c}\text { Recre- } \\
\text { ational } \\
\text { zone }\end{array}$ & $\begin{array}{l}\text { Stationary } \\
\text { recreation } \\
\text { zone }\end{array}$ & $\begin{array}{l}\text { Econo- } \\
\text { mic zone }\end{array}$ & $\begin{array}{c}\text { Rare, ad- } \\
\text { ventive, } \\
\text { types }\end{array}$ \\
\hline Apiaceae & Cicuta virosa $\mathrm{L}$. & Hel & Per & $\mathrm{HeSc}$ & $\mathrm{MsTr}$ & $\mathrm{Hg}$ & Pal & + & - & - & - & - \\
\hline Apiaceae & Cenolophium denudatum (Homem) Tutin & $\mathrm{HKr}$ & Per & $\mathrm{He}$ & $\operatorname{MgTr}$ & HgMs & $\operatorname{Pr}$ & + & + & - & - & - \\
\hline Apiaceae & Conium maculatum $\mathrm{L}$. & $\mathrm{HKr}$ & Bien & $\mathrm{He}$ & $\mathrm{MsTr}$ & Ms & $\mathrm{Ru}$ & - & - & - & + & - \\
\hline Apiaceae & Daucus carota L. & $\mathrm{HKr}$ & Per & $\mathrm{ScHe}$ & Og-MgTr & $\mathrm{XMs}$ & $\mathrm{Ru}$ & + & + & + & + & - \\
\hline Apiaceae & Eryngium campestre $\mathrm{L}$. & $\mathrm{G}$ & Per & $\mathrm{He}$ & MsTr & $\mathrm{X}$ & RuSt & - & + & + & + & - \\
\hline Apiaceae & E. planum L. & $\mathrm{HKr}$ & Per & $\mathrm{ScHe}$ & Og-MsTr & $\mathrm{XMs}$ & StPr & + & + & - & - & - \\
\hline Apiaceae & Heracleum sibiricum $\mathrm{L}$. & $\mathrm{HKr}$ & Bien & $\mathrm{ScHe}$ & MsTr & Ms & SilPr & + & + & + & + & - \\
\hline Apiaceae & Oenanthe aquatica (L.) Poir. & $\mathrm{HKr}$ & Per & $\mathrm{ScHe}$ & $\mathrm{MsTr}$ & $\mathrm{Hg}$ & $\mathrm{Pal}$ & + & + & + & + & - \\
\hline Apiaceae & Pastinaca sylvestris Mill. & $\mathrm{HKr}$ & Bien & $\mathrm{ScHe}$ & $\mathrm{MsTr}$ & Ms & SilPr & + & - & - & - & - \\
\hline Apiaceae & Peucedanum oreoselinum (L.) Moench. & $\mathrm{HKr}$ & Per & $\mathrm{HeSc}$ & OgTr & $\mathrm{XMs}$ & SilPs & - & + & - & - & - \\
\hline Apiaceae & Seseli libanotis DC. & $\mathrm{HKr}$ & Per & $\mathrm{He}$ & MsTr & $\mathrm{XMs}$ & RuSt & - & + & + & + & - \\
\hline Apiaceae & Sium latifolium L. & $\mathrm{HKr}$ & Per & $\mathrm{ScHe}$ & MsTr & $\mathrm{Hg}$ & $\mathrm{Pal}$ & + & + & + & - & - \\
\hline Apiaceae & S. sisaroideum DC. & $\mathrm{HKr}$ & Per & $\mathrm{He}$ & $\mathrm{MsTr}$ & $\mathrm{Hg}$ & Pal & + & + & - & - & - \\
\hline Apiaceae & Torilis japonijca (Houtt.) DC. & $\mathrm{T}$ & Bien & $\mathrm{ScHe}$ & MsTr & $\mathrm{XMs}$ & RuSil & + & + & - & - & - \\
\hline Aristolochiaceae & Aristolochia clematitis L. & $\mathrm{G}$ & Per & $\mathrm{HeSc}$ & MsTr & HgMs & RuPrSil & + & + & - & - & - \\
\hline Aristolochiaceae & Asarum europaeum L. & $\mathrm{G}$ & Per & $\mathrm{Sc}$ & $\operatorname{MgTr}$ & Ms & Sil & + & - & - & - & RLD3 \\
\hline Asclepiadaceae & Asclepias syriaca L. & $\mathrm{G}$ & Per & $\mathrm{He}$ & MsTr & $\mathrm{XMs}$ & $\mathrm{Ru}$ & - & - & - & + & Adv \\
\hline Asclepiadaceae & Vincetoxicum hirundinaria Medik. & $\mathrm{HKr}$ & Per & $\mathrm{ScHe}$ & $\mathrm{MsTr}$ & MsX & StSMnSil & + & + & - & - & - \\
\hline Asclepiadaceae & V. scandens Sommier et Levier & $\mathrm{HKr}$ & Per & $\mathrm{ScHe}$ & MsTr & Ms & Sil & + & - & - & - & RLD4 \\
\hline Asteraceae & Achillea collina J. Becker ex Rchb. & $\mathrm{HKr}$ & Per & $\mathrm{He}$ & MsTr & $\mathrm{XMs}$ & StPr & + & + & - & - & - \\
\hline Asteraceae & A. micrantha Willd. & $\mathrm{HKr}$ & Per & $\mathrm{ScHe}$ & $\mathrm{Og} T r$ & MsX & SilPs & - & + & - & - & - \\
\hline Asteraceae & A. nobilis L. & $\mathrm{HKr}$ & Per & $\mathrm{He}$ & MsTr & MsX & PrRuSt & - & + & - & - & - \\
\hline Asteraceae & A. submillefolium Klok. et Krytzka & $\mathrm{HKr}$ & Per & $\mathrm{ScHe}$ & $\mathrm{MsTr}$ & $\mathrm{XMs}$ & SilPrSt & + & + & + & + & - \\
\hline Asteraceae & Ambrosia artemisifolia $\mathrm{L}$. & $\mathrm{T}$ & Ann & $\mathrm{ScHe}$ & $\mathrm{OgMgTr}$ & $\mathrm{X}$ & $\mathrm{Ru}$ & - & + & + & + & Adv \\
\hline Asteraceae & Anthemis cotula L. & $\mathrm{T}$ & Ann & $\mathrm{He}$ & MsTr & $\mathrm{XMs}$ & $\mathrm{Ru}$ & + & + & + & + & $\mathrm{Adv}$ \\
\hline Asteraceae & A. ruthenica Bieb. & $\mathrm{T}$ & Ann & $\mathrm{ScHe}$ & $\mathrm{Og} T \mathrm{r}$ & $\mathrm{X}$ & RuSt & - & + & + & + & - \\
\hline Asteraceae & A. subtinctoria Dobrocz. & $\mathrm{HKr}$ & Ann & $\mathrm{ScHe}$ & $\mathrm{MsOgTr}$ & $\mathrm{MsX}$ & RuSt & - & + & - & - & - \\
\hline Asteraceae & Arctium lappa $\mathrm{L}$. & $\mathrm{HKr}$ & Bien & $\mathrm{ScHe}$ & $\mathrm{MgTr}$ & $\mathrm{Ms}$ & SilRu & + & + & + & + & - \\
\hline Asteraceae & A. nemorosum Lej. & $\mathrm{HKr}$ & Bien & $\mathrm{HeSc}$ & $\mathrm{MgTr}$ & Ms & RuSil & + & - & - & - & - \\
\hline Asteraceae & A. minus (Hill.) Bernh. & $\mathrm{HKr}$ & Bien & $\mathrm{ScHe}$ & $\mathrm{MgTr}$ & Ms & $\mathrm{Ru}$ & + & + & - & - & - \\
\hline Asteraceae & A. tomentosum Mill. & $\mathrm{HKr}$ & Bien & $\mathrm{He}$ & $\operatorname{MgTr}$ & Ms & $\mathrm{Ru}$ & + & + & - & - & - \\
\hline Asteraceae & Artemisia abrotamum L. & $\mathrm{Ch}$ & Fr & $\mathrm{He}$ & OgMsTr & HgMs & PalPr & + & - & - & - & - \\
\hline Asteraceae & A. absinthium L. & $\mathrm{HKr}$ & Per & $\mathrm{He}$ & MsTr & $\mathrm{XMs}$ & $\mathrm{Ru}$ & + & + & + & + & Adv \\
\hline Asteraceae & A. austriaca Jacq. & $\mathrm{Ch}$ & Per & $\mathrm{He}$ & $\mathrm{MsTr}$ & $\mathrm{X}$ & RuSt & - & + & + & + & - \\
\hline Asteraceae & A. campestris L. & $\mathrm{HKr}$ & Per & $\mathrm{He}$ & OgMsTr & $\mathrm{X}$ & SilPsSt & - & + & + & - & - \\
\hline Asteraceae & A. vulgaris $\mathrm{L}$. & $\mathrm{HKr}$ & Per & $\mathrm{ScHe}$ & $\operatorname{MgTr}$ & Ms & PrRu & + & + & + & + & - \\
\hline Asteraceae & Aster bessarabicus Bemh. ex Rchb. & $\mathrm{HKr}$ & Per & $\mathrm{ScHe}$ & $\operatorname{MgTr}$ & MsX & SilSt & + & - & - & - & RLD3 \\
\hline Asteraceae & A.novae-angliae $\mathrm{L}$. & $\mathrm{HKr}$ & Per & $\mathrm{ScHe}$ & $\operatorname{MgTr}$ & Ms & Cul & - & - & - & + & Adv \\
\hline Asteraceae & A. salignus Willd. & $\mathrm{HKr}$ & Per & $\mathrm{He}$ & MsTr & Ms & Cul & - & - & - & + & Adv \\
\hline Asteraceae & Bidens tripartita L. & $\mathrm{T}$ & Ann & $\mathrm{ScHe}$ & MsTr & HgMs & PalPr & + & + & + & + & - \\
\hline Asteraceae & Calendula officinalis $\mathrm{L}$. & $\mathrm{T}$ & Ann & $\mathrm{ScHe}$ & $\mathrm{MsTr}$ & Ms & $\mathrm{Cul}$ & - & - & - & + & Adv \\
\hline Asteraceae & Carduus acanthoides $\mathrm{L}$. & $\mathrm{HKr}$ & Bien & $\mathrm{He}$ & MsTr & MsX & PrStRu & + & + & + & + & - \\
\hline Asteraceae & C. crispus $\mathrm{L}$. & $\mathrm{HKr}$ & Bien & $\mathrm{ScHe}$ & MsTr & Ms & SilRu & + & + & - & - & - \\
\hline Asteraceae & C. nutans $\mathrm{L}$. & $\mathrm{HKr}$ & Bien & $\mathrm{He}$ & $\mathrm{MgTr}$ & MsX & StRu & - & + & - & - & Adv \\
\hline Asteraceae & Centaurea cyamus L. & $\mathrm{HKr}$ & Bien & $\mathrm{He}$ & MsTr & MsX & $\mathrm{Ru}$ & - & - & - & + & $\mathrm{Adv}$ \\
\hline Asteraceae & C. diffusa Lam. & $\mathrm{HKr}$ & Bien & $\mathrm{He}$ & $\mathrm{MsTr}$ & $\mathrm{X}$ & $\mathrm{StRu}$ & - & + & + & + & Adv \\
\hline Asteraceae & C. jacea L. & $\mathrm{HKr}$ & Per & $\mathrm{ScHe}$ & $\mathrm{MgTr}$ & Ms & Pr & + & + & - & - & - \\
\hline Asteraceae & C. scabiosa L. & $\mathrm{HKr}$ & Per & $\mathrm{ScHe}$ & $\operatorname{MgTr}$ & $\mathrm{MsX}$ & $\mathrm{StRu}$ & - & + & + & - & - \\
\hline Asteraceae & C. trichocephala M. Bieb. & $\mathrm{HKr}$ & Per & $\mathrm{ScHe}$ & MsTr & XMs & PrSt & + & + & - & - & - \\
\hline Asteraceae & Chondrilla juncea $\mathrm{L}$. & $\mathrm{HKr}$ & Per & $\mathrm{He}$ & OgTr & MsX & RuStPs & - & + & + & + & - \\
\hline Asteraceae & Cichorium inthybus $\mathrm{L}$. & $\mathrm{HKr}$ & Per & $\mathrm{He}$ & MsTr & MsX & RuStPr & + & + & + & + & Adv \\
\hline Asteraceae & Cirsium canum (L.) All. & $\mathrm{HKr}$ & Per & $\mathrm{He}$ & AlkTr & Ms & PrHal & + & + & - & - & - \\
\hline Asteraceae & C. incanum (S. G. Gmel.) Fisch. & G & Per & $\mathrm{He}$ & $\operatorname{MgTr}$ & $\mathrm{MsHg}$ & $\mathrm{RuPr}$ & + & + & + & + & - \\
\hline Asteraceae & C. setosum (Willd.) Bess. & G & Per & $\mathrm{He}$ & $\mathrm{Ms} \operatorname{Tr}$ & MsX & $\mathrm{Ru}$ & + & + & + & + & - \\
\hline Asteraceae & C. ucrainicum Bess. & $\mathrm{HKr}$ & Bien & $\mathrm{He}$ & OgMsTr & $\mathrm{X}$ & RuSt & + & + & + & + & - \\
\hline Asteraceae & C. vulgare (Savit) Ten. & $\mathrm{HKr}$ & Bien & $\mathrm{ScHe}$ & $\mathrm{MgTr}$ & $\mathrm{XMs}$ & $\mathrm{Ru}$ & + & + & + & + & - \\
\hline Asteraceae & Coniza canadensis (L.) Cronq. & $\mathrm{HKr}$ & Bien & $\mathrm{ScHe}$ & OgMgTr & MsX & $\mathrm{Ru}$ & + & + & + & + & Adv \\
\hline Asteraceae & Crepis rhoedifolia M. Bieb. & $\mathrm{T}$ & Ann & $\mathrm{He}$ & MsTr & $\mathrm{MsX}$ & $\mathrm{StRu}$ & - & + & + & + & - \\
\hline Asteraceae & C. tectorum $\mathrm{L}$. & $\mathrm{HKr}$ & Bien & $\mathrm{He}$ & OgMsTr & $\mathrm{MsX}$ & PsStRu & + & + & + & + & - \\
\hline Asteraceae & Echinops sphaerocephalus L. & $\mathrm{HKr}$ & Per & $\mathrm{ScHe}$ & MsTr & $\mathrm{X}$ & RuPrSt & + & - & - & - & - \\
\hline Asteraceae & Erigeron acris L. & $\mathrm{HKr}$ & Bien & $\mathrm{ScHe}$ & $\mathrm{MsTr}$ & MsX & $\mathrm{RuPrSt}$ & + & + & + & + & - \\
\hline Asteraceae & E. podolicus Bess. & $\mathrm{HKr}$ & Bien & $\mathrm{He}$ & OgMsTr & $\mathrm{XMs}$ & $\mathrm{StPr}$ & + & + & + & + & - \\
\hline Asteraceae & Eupatorium cannabinum $\mathrm{L}$. & $\mathrm{HKr}$ & Per & $\mathrm{ScHe}$ & $\operatorname{MgTr}$ & HgMs & SilPr & + & + & - & - & - \\
\hline Asteraceae & Filago arvensis L. & $\mathrm{T}$ & Ann & $\mathrm{ScHe}$ & OgMsTr & $\mathrm{MsX}$ & PsStRu & + & + & - & - & - \\
\hline Asteraceae & Galinsoga parviflora Cav. & $\mathrm{T}$ & Ann & $\mathrm{ScHe}$ & MsTr & Ms & $\mathrm{Ru}$ & - & - & - & + & Adv \\
\hline Asteraceae & Grindelia squarrosa (Pursh) Dunal. & $\mathrm{HKr}$ & Per & $\mathrm{He}$ & Og-MgTr & MsX & $\mathrm{Ru}$ & - & - & - & + & Adv \\
\hline Asteraceae & Helianthus anmuus L. & $\mathrm{T}$ & Ann & $\mathrm{He}$ & $\operatorname{MgTr}$ & $\mathrm{XMs}$ & $\mathrm{Cu}$ & - & - & - & + & Adv \\
\hline Asteraceae & H. tuberosus L. & $\mathrm{G}$ & Per & $\mathrm{He}$ & $\operatorname{MgTr}$ & $\mathrm{XMs}$ & $\mathrm{Cu}$ & - & - & - & + & Adv \\
\hline Asteraceae & Helishrysum arenarium (L.) Moench. & $\mathrm{HKr}$ & Per & $\mathrm{He}$ & OgTr & MsX & StPs & + & + & + & + & - \\
\hline Asteraceae & Heliopsis scabra Dunal. & G & Per & $\mathrm{ScHe}$ & MsTr & $\mathrm{XMs}$ & $\mathrm{Cu}$ & - & - & - & + & Adv \\
\hline Asteraceae & Hieracium umbellatum $\mathrm{L}$. & $\mathrm{HKr}$ & Per & $\mathrm{ScHe}$ & $\mathrm{OgTr}$ & $\mathrm{MsX}$ & StPs & + & + & + & + & - \\
\hline Asteraceae & Inula britanica $\mathrm{L}$. & $\mathrm{HKr}$ & Per & $\mathrm{He}$ & $\mathrm{MsTr}$ & Ms & $\mathrm{RuPr}$ & + & + & - & - & - \\
\hline Asteraceae & I. germanica L. & $\mathrm{G}$ & Per & $\mathrm{He}$ & $\mathrm{MgTr}$ & XMs & StPr & + & + & - & - & - \\
\hline Asteraceae & I. helenium L. & $\mathrm{HKr}$ & Per & $\mathrm{He}$ & $\operatorname{MgTr}$ & HgMs & $\operatorname{Pr}$ & + & - & - & - & RLD3 \\
\hline Asteraceae & I. salicina L. & $\mathrm{G}$ & Per & $\mathrm{ScHe}$ & $\mathrm{OgTr}$ & $\mathrm{XMs}$ & PrSMn & + & - & - & - & - \\
\hline Asteraceae & Iva xanthiifolia Nutt. & $\mathrm{T}$ & Ann & $\mathrm{ScHe}$ & OgMgTr & $\mathrm{XMs}$ & $\mathrm{Ru}$ & - & + & + & + & Adv \\
\hline Asteraceae & Jurinea longifolia DC. & $\mathrm{HKr}$ & Per & $\mathrm{He}$ & $\mathrm{OgTr}$ & MsX & StPs & + & - & - & - & RLD2 \\
\hline Asteraceae & Lactuca chaixii Vill. & $\mathrm{T}$ & Bien & $\mathrm{HeSc}$ & $\operatorname{MgTr}$ & Ms & Sil & + & - & - & - & - \\
\hline Asteraceae & L. serriola Tomer & $\mathrm{HKr}$ & Bien & $\mathrm{He}$ & MsTr & $\mathrm{XMs}$ & $\mathrm{Ru}$ & + & + & + & + & Adv \\
\hline Asteraceae & L. tatarica (L.) C. A. Mey. & $\mathrm{G}$ & Per & $\mathrm{He}$ & $\mathrm{MsTr}$ & $\mathrm{XMs}$ & $\mathrm{HalRu}$ & + & + & + & + & - \\
\hline
\end{tabular}




\begin{tabular}{|c|c|c|c|c|c|c|c|c|c|c|c|c|}
\hline Families & Species & $\begin{array}{l}\text { Clima- } \\
\text { morphs }\end{array}$ & $\begin{array}{l}\text { Bio- } \\
\text { morphs }\end{array}$ & $\begin{array}{l}\text { Helio- } \\
\text { morphs }\end{array}$ & $\begin{array}{l}\text { Tropho- } \\
\text { morphs }\end{array}$ & $\begin{array}{l}\text { Hygro- } \\
\text { morphs }\end{array}$ & $\begin{array}{l}\text { Ceno- } \\
\text { morphs }\end{array}$ & $\begin{array}{l}\text { Protec- } \\
\text { ted zone }\end{array}$ & $\begin{array}{c}\text { Recre- } \\
\text { ational } \\
\text { zone }\end{array}$ & $\begin{array}{c}\text { Stationary } \\
\text { recreation } \\
\text { zone }\end{array}$ & $\begin{array}{l}\text { Econo- } \\
\text { mic zone }\end{array}$ & $\begin{array}{c}\text { Rare, ad- } \\
\text { ventive, } \\
\text { types }\end{array}$ \\
\hline Asteraceae & Lapsana communis L. & $\mathrm{T}$ & Ann & $\mathrm{HeSc}$ & $\operatorname{MgTr}$ & XMs & RuSil & + & + & - & - & - \\
\hline Asteraceae & Leontodon autumnalis L. & $\mathrm{HKr}$ & Per & $\mathrm{ScHe}$ & MsTr & Ms & $\mathrm{RuPr}$ & + & + & - & - & - \\
\hline Asteraceae & Lepidotheca suaveolens (Pursh) Nutt. & $\mathrm{T}$ & Ann & $\mathrm{He}$ & $\operatorname{MsTr}$ & MsX & $\mathrm{Ru}$ & + & + & + & + & Adv \\
\hline Asteraceae & Matricaria recutita $\mathrm{L}$. & $\mathrm{T}$ & Ann & $\mathrm{He}$ & $\operatorname{MsTr}$ & MsX & $\mathrm{Ru}$ & + & + & + & + & Adv \\
\hline Asteraceae & Onopordum acanthium L. & $\mathrm{HKr}$ & Bien & $\mathrm{He}$ & $\operatorname{MsTr}$ & MsX & $\mathrm{Ru}$ & - & + & + & + & Adv \\
\hline Asteraceae & Petasites spurius (Retz.) Rchb. & $\mathrm{G}$ & Per & $\mathrm{ScHe}$ & $\mathrm{Og} \operatorname{Tr}$ & $\mathrm{MsHg}$ & Ps & + & - & - & - & Adv \\
\hline Asteraceae & Phalacroloma annuum (L.) Dumort. & $\mathrm{HKr}$ & Bien & $\mathrm{ScHe}$ & MsTr & MsX & $\mathrm{Ru}$ & - & - & + & + & Adv \\
\hline Asteraceae & Picris hieracioides L. & $\mathrm{HKr}$ & Per & $\mathrm{He}$ & MsTr & MsX & RuSt & + & + & + & + & - \\
\hline Asteraceae & Pilosellaechioides (Lumn.)F. Schultz etSch. & $\mathrm{HKr}$ & Per & $\mathrm{ScHe}$ & OgMsTr & MsX & SilPsSt & - & + & + & + & - \\
\hline Asteraceae & P. floribunda (Wimmer et Grab.) Fr. & $\mathrm{HKr}$ & Per & $\mathrm{He}$ & MsTr & $\mathrm{HgMs}$ & $\mathrm{RuPr}$ & + & + & - & - & - \\
\hline Asteraceae & P. officinarum F.Schultz. et Sch. & $\mathrm{HKr}$ & Per & $\mathrm{He}$ & OgMsTr & XMs & PsSilPr & + & + & - & - & - \\
\hline Asteraceae & Pulicaria vulgaris Gaertn. & $\mathrm{T}$ & Ann & $\mathrm{He}$ & AlkMsTr & $\mathrm{MsHg}$ & $\mathrm{RuPr}$ & + & + & - & - & - \\
\hline Asteraceae & Rutbeckia hirta L. & $\mathrm{T}$ & Ann & $\mathrm{He}$ & $\operatorname{MsTr}$ & Ms & Cul & - & - & - & + & Adv \\
\hline Asteraceae & Scorzonera laciniata $\mathrm{L}$. & $\mathrm{HKr}$ & Per & $\mathrm{He}$ & AlkTr & $\mathrm{X}-\mathrm{Ms}$ & HalStPr & - & + & - & - & - \\
\hline Asteraceae & S. parviflora Jacq. & $\mathrm{HKr}$ & Per & $\mathrm{He}$ & AlkTr & Ms & HalPr & - & + & - & - & - \\
\hline Asteraceae & $\begin{array}{l}\text { Senecio borysthenicus (DC.) Andrz. ex } \\
\text { Czem. }\end{array}$ & $\mathrm{HKr}$ & Per & $\mathrm{ScHe}$ & $\mathrm{Og} \operatorname{Tr}$ & $\mathrm{XMs}$ & Ps & - & - & - & - & $\begin{array}{l}\text { ERLR, } \\
\text { RLD3 }\end{array}$ \\
\hline Asteraceae & S. jacobaea L. & $\mathrm{HKr}$ & Per & $\mathrm{He}$ & $\operatorname{Ms} T r$ & MsX & SMnPrSt & + & + & - & - & - \\
\hline Asteraceae & S. vernalis Waldst. et Kit. & $\mathrm{T}$ & Ann & $\mathrm{ScHe}$ & Og-MgTr & $\mathrm{XMs}$ & $\mathrm{Ru}$ & - & + & + & + & - \\
\hline Asteraceae & S. vulgaris L. & $\mathrm{T}$ & Ann & $\mathrm{He}$ & $\mathrm{MsTr}$ & Ms & $\mathrm{Ru}$ & - & + & + & + & Adv \\
\hline Asteraceae & Serratula coronata L. & $\mathrm{HKr}$ & Per & $\mathrm{ScHe}$ & MsTr & Ms & $\mathrm{SMnPr}$ & + & - & - & - & - \\
\hline Asteraceae & Solidago canadensis L. & $\mathrm{HKr}$ & Per & $\mathrm{ScHe}$ & $\mathrm{MsTr}$ & XMs & RuCul & - & - & - & + & Adv \\
\hline Asteraceae & Sonchus arvensis L. & $\mathrm{G}$ & Per & $\mathrm{He}$ & $\operatorname{MgTr}$ & $\mathrm{XMs}$ & $\operatorname{PrRu}$ & - & + & + & + & Adv \\
\hline Asteraceae & S. oleraceus L. & $\mathrm{T}$ & Ann & $\mathrm{He}$ & MsTr & $\mathrm{XMs}$ & $\mathrm{Ru}$ & - & + & + & + & $\mathrm{Adv}$ \\
\hline Asteraceae & S. palustris L. & $\mathrm{HKr}$ & Per & $\mathrm{He}$ & $\mathrm{MsTr}$ & $\mathrm{MsHg}$ & Pal & + & - & - & - & - \\
\hline Asteraceae & Tagetes patula $\mathrm{L}$. & $\mathrm{T}$ & Ann & $\mathrm{He}$ & $\mathrm{MgTr}$ & $\mathrm{MsX}$ & $\mathrm{Cul}$ & - & - & - & + & Adv \\
\hline Asteraceae & Tanacetum vulgare $\mathrm{L}$. & $\mathrm{HKr}$ & Per & $\mathrm{He}$ & OgMgTr & MsX & StRuPr & + & + & + & - & - \\
\hline Asteraceae & Taraxacum officinale Wigg. aggr. & $\mathrm{HKr}$ & Per & $\mathrm{ScHe}$ & MsTr & Ms & $\operatorname{RuPr}$ & + & + & + & + & - \\
\hline Asteraceae & Tragopogon borystenicus Artemcz. & $\mathrm{HKr}$ & Bien & $\mathrm{He}$ & $\mathrm{OgTr}$ & MsX & PsSt & + & - & - & - & $\begin{array}{l}\text { RLD 0, } \\
\text { ERLI }\end{array}$ \\
\hline Asteraceae & T. major Jacq. & $\mathrm{HKr}$ & Bien & $\mathrm{He}$ & $\operatorname{Ms} T r$ & MsX & SMnRuSt & + & + & + & - & - \\
\hline Asteraceae & Tripleurospermum inodorum (L.) Sch. & $\mathrm{HKr}$ & Bien & $\mathrm{He}$ & $\operatorname{MgTr}$ & MsX & $\mathrm{Ru}$ & + & + & + & - & Adv \\
\hline Asteraceae & Tripolium pannonicum (Jacq.) Dobrocz. & $\mathrm{HKr}$ & Bien & $\mathrm{He}$ & Alk & $\mathrm{MsHg}$ & PalPrH & + & + & - & - & - \\
\hline Asteraceae & Tussilago farfara $\mathrm{L}$. & G & Per & $\mathrm{He}$ & $\operatorname{MsTr}$ & $\mathrm{MsHg}$ & $\mathrm{RuPr}$ & + & + & + & - & - \\
\hline Asteraceae & Xantium albinum (Widd.) H. Scholtz & $\mathrm{T}$ & Ann & $\mathrm{He}$ & Og-MgTr & $\begin{array}{l}\text { XMs- } \\
\mathrm{MsHg}\end{array}$ & $\mathrm{Ru}$ & + & + & + & + & Adv \\
\hline Asteraceae & X. strumarium $\mathrm{L}$. & $\mathrm{T}$ & Ann & $\mathrm{He}$ & $\operatorname{MsTr}$ & $\begin{array}{l}\text { HgMs- } \\
\text { XMs }\end{array}$ & $\mathrm{Ru}$ & + & + & + & + & Adv \\
\hline Berberidaceae & Mahonia aquifolium Nutt. & $\mathrm{nPh}$ & $\mathrm{Fr}$ & $\mathrm{HeSc}$ & OgMgTr & Ms & $\mathrm{SilCu}$ & - & - & - & + & Adv \\
\hline Betulaceae & Alnus glutinosa (L.) Gaerthn. & $\mathrm{Ph}$ & Arb & $\mathrm{ScHe}$ & $\operatorname{MgTr}$ & $\mathrm{Hg}$ & PalSil & + & - & - & - & RLD3 \\
\hline Betulaceae & Betula pubescens Ehrh. & $\mathrm{Ph}$ & Arb & $\mathrm{ScHe}$ & $\mathrm{MsOgTr}$ & $\mathrm{Hg}$ & Sil & - & - & - & + & - \\
\hline Boraginaceae & Aegonychon purpureocaenuleum (L.)Holub & $\mathrm{HKr}$ & Per & $\mathrm{HeSc}$ & $\operatorname{MsMgTr}$ & Ms & Sil & + & - & - & - & RLD 3 \\
\hline Boraginaceae & Anchusa officinalis L & $\mathrm{HKr}$ & Bien & $\mathrm{ScHe}$ & $\mathrm{OgTr}$ & MsX & RuSilPs & + & + & + & - & Adv \\
\hline Boraginaceae & Asperugo procumbens L. & $\mathrm{T}$ & Ann & $\mathrm{ScHe}$ & $\mathrm{MsTr}$ & $\mathrm{XMs}$ & $\mathrm{Ru}$ & - & + & + & + & - \\
\hline Boraginaceae & Buglossoides arvensis (L.) I. M. Johnst. & $\mathrm{HKr}$ & Ann & $\mathrm{ScHe}$ & MsTr & MsX & $\mathrm{Ru}$ & + & + & + & - & Adv \\
\hline Boraginaceae & Cynoglossum officinale L. & $\mathrm{G}$ & Ann & $\mathrm{He}$ & $\operatorname{OgTr}$ & $\mathrm{XMs}$ & $\mathrm{Ru}$ & - & + & + & + & $\mathrm{Adv}$ \\
\hline Boraginaceae & Lappula squarrosa (Retz) Dumort. & $\mathrm{HKr}$ & Bien & $\mathrm{He}$ & OgMsTr & MsX & RuSt & - & - & - & + & Adv \\
\hline Boraginaceae & Myosotis arvensis (L.) Hill & $\mathrm{T}$ & Ann & $\mathrm{He}$ & MsTr & MsX & $\mathrm{Ru}$ & - & + & + & + & Adv \\
\hline Boraginaceae & $\begin{array}{l}\text { M. laxa Lehm. (M. caespitosa } \\
\text { K. P. Suchultz) }\end{array}$ & $\mathrm{HKr}$ & Ann & $\mathrm{ScHe}$ & MsTr & MsX & PalPr & + & + & - & - & - \\
\hline Boraginaceae & M. micrantha Pall.ex Lehm. & $\mathrm{HKr}$ & Per & $\mathrm{He}$ & $\mathrm{Ms} T r$ & MsX & $\mathrm{Ru}$ & - & - & + & + & - \\
\hline Boraginaceae & M. nemorosa Besser & $\mathrm{HKr}$ & Per & $\mathrm{ScHe}$ & $\mathrm{Ms} T r$ & $\mathrm{MsHg}$ & SilPr & + & - & - & - & - \\
\hline Boraginaceae & M. scorpioides $\mathrm{L}$. & $\mathrm{HKr}$ & Per & $\mathrm{ScHe}$ & $\mathrm{MsTr}$ & $\mathrm{Hg}$ & PrPal & + & + & - & - & - \\
\hline Boraginaceae & M. sparsiflora J.C. Mikan ex Pohl & $\mathrm{HKr}$ & Ann & $\mathrm{ScHe}$ & MsTr & $\mathrm{MsX}$ & RuSil & + & + & - & - & - \\
\hline Boraginaceae & Pulmonaria obscura Dumort. & $\mathrm{HKr}$ & Per & $\mathrm{HeSc}$ & MsTr & Ms & Sil & + & - & - & - & - \\
\hline Boraginaceae & Symphytum officinale L. & $\mathrm{HKr}$ & Per & $\mathrm{ScHe}$ & $\operatorname{MgTr}$ & $\mathrm{MsHg}$ & PalPr & + & + & - & - & - \\
\hline Brassicaceae & Alliaria petiolata (Bieb.) Cavara et Grande & $\mathrm{HKr}$ & Per & $\mathrm{HeSc}$ & MsTr & $\mathrm{XMs}$ & RuSil & + & + & - & - & - \\
\hline Brassicaceae & Alyssum desertorum Stapf. & $\mathrm{T}$ & Ann & $\mathrm{He}$ & MsTr & MsX & RuSt & - & + & + & + & - \\
\hline Brassicaceae & Arabidopsis thaliana (L.) Heynh. & $\mathrm{HKr}$ & Ann & $\mathrm{He}$ & $\mathrm{Og} \operatorname{Tr}$ & XMs & PsRu & - & + & - & - & Adv \\
\hline Brassicaceae & Barbarea stricta Andrz. & $\mathrm{HKr}$ & Ann & $\mathrm{ScHe}$ & $\operatorname{MgTr}$ & $\mathrm{HgMs}$ & PalPr & + & - & - & - & - \\
\hline Brassicaceae & B. vulgaris $\mathrm{R} . \mathrm{Br}$. & $\mathrm{HKr}$ & Ann & $\mathrm{ScHe}$ & MsMgTr & Ms & PrRu & + & + & - & - & - \\
\hline Brassicaceae & B. incana (L.) DC. & $\mathrm{HKr}$ & Bien & $\mathrm{ScHe}$ & Og-MgTr & $\mathrm{XMs}$ & $\mathrm{Ru}$ & + & + & + & + & - \\
\hline Brassicaceae & Bunias orientalis L. & $\mathrm{HKr}$ & Bien & $\mathrm{ScHe}$ & OgMgTr & $\mathrm{XMs}$ & $\mathrm{Ru}$ & + & + & - & - & - \\
\hline Brassicaceae & Camelina microcarpa Andrcz. & $\mathrm{T}$ & Ann & $\mathrm{He}$ & $\mathrm{MsTr}$ & $\mathrm{XMs}$ & SilRu & + & + & - & - & Adv \\
\hline Brassicaceae & Capsella bursa-pastoris (L.) Medik. & $\mathrm{T}$ & Ann & $\mathrm{He}$ & $\mathrm{MsTr}$ & $\mathrm{XMs}$ & $\mathrm{Ru}$ & + & + & + & + & Adv \\
\hline Brassicaceae & Cardaria draba (L.) Desv. & G & Per & $\mathrm{He}$ & $\mathrm{MsTr}$ & MsX & $\mathrm{Ru}$ & - & - & - & + & Adv \\
\hline Brassicaceae & Chorispora tenella (Pall.) DC. & $\mathrm{T}$ & Ann & $\mathrm{He}$ & MsTr & MsX & $\mathrm{Ru}$ & + & + & + & + & Adv \\
\hline Brassicaceae & Descurainia sophia (L.) Webb et Plantl & $\mathrm{T}$ & Ann & $\mathrm{He}$ & MsTr & $\mathrm{XMs}$ & $\mathrm{Ru}$ & - & + & + & + & Adv \\
\hline Brassicaceae & Diplotaxis muralis (L.) DC. & $\mathrm{HKr}$ & Ann & $\mathrm{He}$ & $\operatorname{MsTr}$ & MsX & $\mathrm{PtRu}$ & - & + & + & + & Adv \\
\hline Brassicaceae & D. tenuifolia (L.) DC. & $\mathrm{HKr}$ & Per & $\mathrm{He}$ & MsTr & MsX & $\mathrm{Ru}$ & - & + & + & - & Adv \\
\hline Brassicaceae & Draba nemorosa L. & $\mathrm{T}$ & Ann & $\mathrm{He}$ & $\operatorname{MsTr}$ & $\mathrm{XMs}$ & $\mathrm{Ru}$ & + & + & - & - & - \\
\hline Brassicaceae & Erophila verna (L.) Bess. & $\mathrm{T}$ & Ann & $\mathrm{He}$ & OgMsTr & $\mathrm{XMs}$ & RuPsSt & - & + & + & - & - \\
\hline Brassicaceae & Erysimum cheiranthoides L. & $\mathrm{HKr}$ & Bien & $\mathrm{He}$ & $\mathrm{MsTr}$ & Ms & $\mathrm{Ru}$ & - & + & + & - & Adv \\
\hline Brassicaceae & E. diffusum Ehrh & $\mathrm{HKr}$ & Bien & $\mathrm{He}$ & $\mathrm{MsTr}$ & $\mathrm{X}$ & RuSt & - & + & + & + & - \\
\hline Brassicaceae & Hesperidium tristis (L.) G. Beck & $\mathrm{HKr}$ & Bien & $\mathrm{He}$ & MsMgTr & MsX & PrSt & + & - & - & - & RLD 3 \\
\hline Brassicaceae & Hesperis sibirica $\mathrm{L}$. & $\mathrm{HKr}$ & Bien & $\mathrm{HeSc}$ & MsMgTr & Ms & Sil & + & - & - & - & Adv \\
\hline Brassicaceae & Lepidium latifolium $\mathrm{L}$. & $\mathrm{HKr}$ & Per & $\mathrm{He}$ & Og-MgTr & Ms & PrH & + & - & - & - & - \\
\hline Brassicaceae & L. ruderale $\mathrm{L}$. & $\mathrm{HKr}$ & Ann & $\mathrm{He}$ & MsTr & MsX & $\mathrm{Ru}$ & - & - & - & + & Adv \\
\hline Brassicaceae & Microthlaspi perfoliata (L.) F. K. Meyer & $\mathrm{T}$ & Ann & $\mathrm{He}$ & MsTr & $\mathrm{XMs}$ & PrStRu & - & + & + & + & Adv \\
\hline Brassicaceae & Rorippa amphibia (L.) Bess. & G & Per & $\mathrm{HeSc}$ & $\operatorname{MgTr}$ & HyHg & AqPal & + & + & - & - & - \\
\hline
\end{tabular}




\begin{tabular}{|c|c|c|c|c|c|c|c|c|c|c|c|c|}
\hline Families & Species & $\begin{array}{l}\text { Clima- } \\
\text { morphs }\end{array}$ & $\begin{array}{c}\text { Bio- } \\
\text { morphs }\end{array}$ & $\begin{array}{l}\text { Helio- } \\
\text { morphs }\end{array}$ & $\begin{array}{l}\text { Tropho- } \\
\text { morphs }\end{array}$ & $\begin{array}{l}\text { Hygro- } \\
\text { morphs }\end{array}$ & $\begin{array}{l}\text { Ceno- } \\
\text { morphs }\end{array}$ & $\begin{array}{l}\text { Protec- } \\
\text { ted zone }\end{array}$ & $\begin{array}{c}\text { Recre- } \\
\text { ational } \\
\text { zone }\end{array}$ & $\begin{array}{l}\text { Stationary } \\
\text { recreation } \\
\text { zone }\end{array}$ & $\begin{array}{l}\text { Econo- } \\
\text { mic zone }\end{array}$ & $\begin{array}{c}\text { Rare, ad- } \\
\text { ventive, } \\
\text { types }\end{array}$ \\
\hline Brassicaceae & R. austriaca (Crantz) Bess. & $\mathrm{HKr}$ & Per & $\mathrm{He}$ & Og-MsTr & HgMs & RuPalPr & + & + & - & - & - \\
\hline Brassicaceae & Sinapis arvensis L. & $\mathrm{T}$ & Ann & $\mathrm{He}$ & $\operatorname{MgTr}$ & MsX & $\mathrm{Ru}$ & - & + & + & + & Adv \\
\hline Brassicaceae & Sisymbrium altissimum $\mathrm{L}$. & $\mathrm{HKr}$ & Ann & $\mathrm{He}$ & MsTr & $\mathrm{XMs}$ & $\mathrm{Ru}$ & - & - & + & + & Adv \\
\hline Brassicaceae & S. loeselii L. & $\mathrm{HKr}$ & Ann & $\mathrm{He}$ & OgMsTr & MsX & $\mathrm{Ru}$ & - & + & + & + & Adv \\
\hline Brassicaceae & S. polymorphum (Murray) Roth & $\mathrm{HKr}$ & Ann & $\mathrm{ScHe}$ & MsTr & MsX & RuSMnSt & - & + & + & + & Adv \\
\hline Brassicaceae & Syrenia cana (Pall.et Mitt.) Neilr. & $\mathrm{HKr}$ & Bien & $\mathrm{He}$ & OgTr & MsX & Ps & + & - & - & - & RLD3 \\
\hline Brassicaceae & Thlaspi arvense $\mathrm{L}$. & $\mathrm{T}$ & Ann & $\mathrm{He}$ & MsTr & MsX & $\mathrm{Ru}$ & - & + & + & + & Adv \\
\hline Brassicaceae & Turritis glabra L. & $\mathrm{HKr}$ & Ann & $\mathrm{ScHe}$ & MsTr & Ms & RuSMn & + & + & - & - & - \\
\hline Caesalpiniaceae & Gleditschia triacanthos L. & $\mathrm{Ph}$ & Arb & $\mathrm{He}$ & $\mathrm{MsTr}$ & MsX & $\mathrm{SilCu}$ & - & + & + & + & Adv \\
\hline Campanulaceae & Campanula bononiensis $\mathrm{L}$. & $\mathrm{HKr}$ & Per & $\mathrm{ScHe}$ & $\mathrm{MsTr}$ & MsX & RuStSil & + & + & - & - & - \\
\hline Campanulaceae & C. glomerata $\mathrm{L}$. & $\mathrm{HKr}$ & Per & $\mathrm{ScHe}$ & $\mathrm{MsTr}$ & $\mathrm{XMs}$ & StPrSil & + & - & - & - & RLD3 \\
\hline Campanulaceae & C. persicifolia $\mathrm{L}$. & $\mathrm{HKr}$ & Per & $\mathrm{HeSc}$ & MsTr & Ms & PsSil & + & - & - & - & RLD2 \\
\hline Campanulaceae & C. rapunculoides $\mathrm{L}$. & $\mathrm{HKr}$ & Per & $\mathrm{ScHe}$ & $\mathrm{MsTr}$ & $\mathrm{XMs}$ & StPrSil & + & - & - & - & - \\
\hline Campanulaceae & C. rapunculus L. & $\mathrm{HKr}$ & Bien & $\mathrm{ScHe}$ & $\mathrm{MsTr}$ & Ms & $\mathrm{SMnPr}$ & + & - & - & - & - \\
\hline Campanulaceae & C. sibirica $\mathrm{L}$. & $\mathrm{HKr}$ & Bien & $\mathrm{He}$ & $\mathrm{MsTr}$ & MsX & RuPrSt & + & + & - & - & - \\
\hline Campanulaceae & C. trachelium $\mathrm{L}$. & $\mathrm{HKr}$ & Per & $\mathrm{HeSc}$ & $\mathrm{MsTr}$ & Ms & SMnSil & + & - & - & - & RLD3 \\
\hline Cannabaceae & Cannabis ruderalis Janisch. & $\mathrm{T}$ & Ann & $\mathrm{He}$ & MsTr & MsX & $\mathrm{Ru}$ & - & - & - & + & Adv \\
\hline Cannabaceae & Humulus lupulus L. & $\mathrm{G}$ & Per & $\mathrm{HeSc}$ & MsTr & XMs-Hg & RuSil & + & + & + & + & - \\
\hline Caprifoliaceae & Lonicera tatarica L. & $\mathrm{nPh}$ & $\mathrm{Fr}$ & $\mathrm{ScHe}$ & $\mathrm{MsTr}$ & MsX & $\mathrm{SMnCu}$ & - & - & + & + & Adv \\
\hline Caprifoliaceae & Sambucus nigra L. & $\mathrm{nPh}$ & $\mathrm{Fr}$ & $\mathrm{ScHe}$ & MgMsTr & Ms & RuSil & + & + & + & + & - \\
\hline Caprifoliaceae & Viburnum opulus L. & $\mathrm{nPh}$ & $\mathrm{Fr}$ & $\mathrm{HeSc}$ & MgTr & Ms & Sil & - & - & - & + & - \\
\hline Caryophyllaceae & Alsine media $\mathrm{L}$. & $\mathrm{HKr}$ & Ann & $\mathrm{ScHe}$ & MsMgTr & $\mathrm{HgMs}$ & SilRu & + & + & + & + & - \\
\hline Caryophyllaceae & Arenaria uralensis Pall. ex Spreng. & $\mathrm{HKr}$ & Ann & $\mathrm{He}$ & OgMsTr & MsX & PsStRu & + & + & - & - & - \\
\hline Caryophyllaceae & Cerastium holosteoides Fries & $\mathrm{HKr}$ & Per & $\mathrm{ScHe}$ & MgMsTr & Ms & $\mathrm{SMnPr}$ & + & - & - & - & - \\
\hline Caryophyllaceae & Coronaria flos-cuculi (L.) Four & $\mathrm{HKr}$ & Per & $\mathrm{ScHe}$ & MsTr & $\mathrm{MsHg}$ & SMnPalPr & + & - & - & - & RLD2 \\
\hline Caryophyllaceae & Cucubalus baccifer $\mathrm{L}$. & $\mathrm{HKr}$ & Per & $\mathrm{HeSc}$ & $\operatorname{MgTr}$ & $\mathrm{HgMs}$ & PrSil & + & - & - & - & - \\
\hline Caryophyllaceae & Dianthus campestris Bieb. & $\mathrm{HKr}$ & Per & $\mathrm{ScHe}$ & OgMsTr & $\mathrm{XMs}$ & PsStSMn & + & + & - & - & - \\
\hline Caryophyllaceae & D. laevigatus (Grun.) Klokov & $\mathrm{HKr}$ & Per & $\mathrm{He}$ & OgTr & MsX & Ps & + & - & - & - & - \\
\hline Caryophyllaceae & D. platyodon Klokov & $\mathrm{HKr}$ & Per & $\mathrm{He}$ & OgTr & MsX & SilPs & + & + & - & - & - \\
\hline Caryophyllaceae & D. stenocalyx Juz. & $\mathrm{HKr}$ & Per & $\mathrm{ScHe}$ & MgMsTr & Ms & $\mathrm{SMnPr}$ & + & - & - & - & - \\
\hline Caryophyllaceae & Dichodon viscidum (M. Bieb.) Holub & $\mathrm{T}$ & Ann & $\mathrm{He}$ & AlkTr & $\mathrm{HgMs}$ & $\mathrm{HalPr}$ & + & + & - & - & - \\
\hline Caryophyllaceae & Elisantae viscosa (L.) Rupr & $\mathrm{HKr}$ & Ann & $\mathrm{He}$ & $\mathrm{MsTr}$ & XMs & PsPtSt & + & - & - & - & - \\
\hline Caryophyllaceae & Eremogone longifolia (M. Bieb.) Fenzl & $\mathrm{HKr}$ & Per & $\mathrm{He}$ & OgMsTr & MsX & HalSt & + & + & - & - & RLD2 \\
\hline Caryophyllaceae & Gypsophila paniculata L. & $\mathrm{HKr}$ & Per & $\mathrm{He}$ & OgMsTr & MsX & PsSt & + & + & - & - & - \\
\hline Caryophyllaceae & Herniaria polygama J. Gay & $\mathrm{T}$ & Ann & $\mathrm{He}$ & $\mathrm{MsOgTr}$ & MsX & RuPs & + & + & - & - & - \\
\hline Caryophyllaceae & Holosteum umbellatum L. & $\mathrm{T}$ & Ann & $\mathrm{He}$ & OgMsTr & XMs & RuPtSt & + & + & - & - & - \\
\hline Caryophyllaceae & Melandrium album (Mill.) Garcke & $\mathrm{HKr}$ & Bien & $\mathrm{ScHe}$ & MsTr & MsX & RuSMnPr & + & + & + & - & - \\
\hline Caryophyllaceae & Moeringia trinervia (L.) Clairv. & $\mathrm{HKr}$ & Ann & $\mathrm{HeSc}$ & OgMsTr & $\mathrm{HgMs}$ & Sil & + & - & - & - & - \\
\hline Caryophyllaceae & Myosoton aquaticum (L.) Moench & $\mathrm{HKr}$ & Per & $\mathrm{ScHe}$ & Og-MsTr & HgMs & PalPr & + & + & + & + & - \\
\hline Caryophyllaceae & Oberna behen (L.) Ikonn. & $\mathrm{HKr}$ & Per & $\mathrm{ScHe}$ & MsTr & XMs & RuSMnPr & + & + & - & - & - \\
\hline Caryophyllaceae & Otites borysthenica (Grun.) Klokov & $\mathrm{HKr}$ & Per & $\mathrm{He}$ & $\mathrm{MsOgTr}$ & $\mathrm{XMs}$ & StPs & + & + & - & - & - \\
\hline Caryophyllaceae & Psammophiliella muralis (L.) Ikonn. & $\mathrm{T}$ & Ann & $\mathrm{He}$ & MsTr & Ms & RuPrSt & + & + & - & - & - \\
\hline Caryophyllaceae & Saponaria officinalis L. & $\mathrm{HKr}$ & Per & $\mathrm{ScHe}$ & OgMsTr & Ms & RuSMnPr & + & + & + & - & Adv \\
\hline Caryophyllaceae & $\begin{array}{l}\text { Saponara ofjctilus L. } \\
\text { Silene chlorantha (Willd.) Ehrh. }\end{array}$ & $\mathrm{HKr}$ & Per & $\mathrm{ScHe}$ & OgMsTr & $\begin{array}{l}\text { MS } \\
\text { XMs }\end{array}$ & PsSilSt & $\begin{array}{l}+ \\
+\end{array}$ & + & + & - & Aav \\
\hline Caryophyllaceae & S. dichotoma Ehrh. & $\mathrm{HKr}$ & Ann & $\mathrm{He}$ & MsTr & $\mathrm{XMs}$ & $\mathrm{StRu}$ & + & + & + & - & - \\
\hline Caryophyllaceae & Stellaria graminea L. & $\mathrm{HKr}$ & Per & $\mathrm{ScHe}$ & OgMsTr & $\begin{array}{l}\text { Alvs } \\
\text { Ms }\end{array}$ & $\mathrm{SMnPr}$ & + & + & - & - & - \\
\hline Caryophyllaceae & S. holostea L. & $\mathrm{HKr}$ & Per & $\mathrm{HeSc}$ & MsTr & Ms & $\begin{array}{l}\text { Svintr } \\
\text { Sil }\end{array}$ & + & + & - & - & - \\
\hline Caryophyllaceae & Steris viscaria (L) Raf. & $\mathrm{HKr}$ & Per & $\mathrm{ScHe}$ & OgMsTr & XMs & PrSMn & + & - & - & - & - \\
\hline Celastraceae & Euonymus europaea $\mathrm{L}$. & $\mathrm{nPh}$ & $\mathrm{Fr}$ & $\mathrm{HeSc}$ & MsTr & Ms & SMnSil & + & + & - & - & - \\
\hline Celastraceae & E. verrucosa Scop. & $\mathrm{nPh}$ & $\mathrm{Fr}$ & $\mathrm{HeSc}$ & MsTr & Ms & SMnSil & + & - & - & - & - \\
\hline Ceratophyllaceae & Ceratophyllum demersum $\mathrm{L}$. & $\mathrm{HKr}$ & Per & $\mathrm{Sc}$ & AlkMgTr & Hyer & $\mathrm{Ag}$ & + & + & + & + & - \\
\hline Ceratophyllaceae & C. submersum L. & $\mathrm{HKr}$ & Per & $\mathrm{Sc}$ & MgTr & Hyer & $\mathrm{Aq}$ & + & - & - & - & - \\
\hline Chenopodiaceae & Atriplex patula L. & $\mathrm{T}$ & Ann & $\mathrm{He}$ & MsTr & $\mathrm{XMs}$ & $\begin{array}{l}\mathrm{Aq} \\
\mathrm{Ru}\end{array}$ & - & - & + & + & - \\
\hline Chenopodiaceae & A.sagittata Borkh & $\begin{array}{l}1 \\
\mathrm{~T}\end{array}$ & Ann & $\mathrm{He}$ & AlkMsTr & $\begin{array}{l}\text { Alvis } \\
\mathrm{MsHg}\end{array}$ & RuHalPr & + & + & + & - & Adv \\
\hline Chenopodiaceae & A. tatarica L. & $\mathrm{T}$ & Ann & $\mathrm{He}$ & MsTr & MsX & RuHal & - & + & + & + & Adv \\
\hline Chenopodiaceae & Chenopodium album $\mathrm{L}$. & $\mathrm{T}$ & Ann & $\mathrm{ScHe}$ & $\mathrm{MsTr}$ & MsX & $\mathrm{Ru}$ & + & + & + & + & - \\
\hline Chenopodiaceae & C. hybridum L. & $\mathrm{T}$ & Ann & $\mathrm{HeSc}$ & $\mathrm{MsTr}$ & $\mathrm{XMs}$ & SilRu & + & - & - & - & Adv \\
\hline Chenopodiaceae & C. polyspermum $\mathrm{L}$. & $\mathrm{T}$ & Ann & $\mathrm{HeSc}$ & OgMsTr & $\mathrm{XMs}$ & RuSMnPs & + & + & + & - & Adv \\
\hline Chenopodiaceae & C. urbicum $\mathrm{L}$. & $\mathrm{T}$ & Ann & $\mathrm{He}$ & MsTr & Ms & $\mathrm{Ru}$ & - & - & + & + & - \\
\hline Chenopodiaceae & Corispermum marschallii Stev. & $\mathrm{T}$ & Ann & $\mathrm{He}$ & OgMsTr & $\mathrm{HgMs}$ & Ps & + & + & - & - & - \\
\hline Chenopodiaceae & Kochia laniflora (S. G. Gmel.) Borb & $\mathrm{T}$ & Ann & $\mathrm{ScHe}$ & OgTr & MsX & RuSilPs & - & + & + & - & Adv \\
\hline Chenopodiaceae & Polvcnemum arvense $\mathrm{L}$. & $\mathrm{T}$ & Ann & $\mathrm{ScHe}$ & OgMsTr & $\mathrm{X}$ & RuPsSt & - & + & - & - & Adv \\
\hline Clusiaceae & Hypericum perforatum $\mathrm{L}$. & $\mathrm{HKr}$ & Per & $\mathrm{ScHe}$ & OgMsTr & $\mathrm{Ms}$ & $\mathrm{SMnPr}$ & + & + & - & - & - \\
\hline Convolvulaceae & Calystegia sepium (L.) R. Br. & $\mathrm{HKr}$ & Per & $\mathrm{ScHe}$ & MsTr & $\mathrm{MsHg}$ & PrPal & + & + & + & - & - \\
\hline Convolvulaceae & Convolvulus arvensis $\mathrm{L}$. & G & Per & $\mathrm{ScHe}$ & MsTr & MsX & $\mathrm{Ru}$ & + & + & + & + & - \\
\hline Comaceae & Swida sanquinea (L.) Opiz. & $\mathrm{Ph}$ & Arb & $\mathrm{HeSc}$ & MsTr & Ms & Sil & + & - & - & - & - \\
\hline Crassulaceae & Hylotelephium polonicum (Bloki) Holub & $\mathrm{G}$ & Per & $\mathrm{ScHe}$ & $\mathrm{MsOgTr}$ & XMs & SilPsPt & + & + & - & - & - \\
\hline Cuscutaceae & Cuscuta europaea $\mathrm{L}$. & $\mathrm{T}$ & Ann & $\mathrm{He}$ & $\begin{array}{l}\text { Par } \\
\text { Pan }\end{array}$ & HgMs & PrSil & + & + & - & - & - \\
\hline Cuscutaceae & C. lupuliformis Krock. & $\mathrm{T}$ & Ann & $\mathrm{He}$ & Par & Ms & SilRu & + & + & - & - & - \\
\hline Dipsacaceae & Knautia arvensis (L.) Coult. & $\mathrm{HKr}$ & Per & $\mathrm{He}$ & MsTr & XMs & PrSMn & + & - & - & - & - \\
\hline Dipsacaceae & Scabiosa ochroleuca L. & $\mathrm{HKr}$ & Per & $\mathrm{ScHe}$ & MsTr & MsX & PsPrSt & + & + & - & - & - \\
\hline Elaeagnaceae & Elaeagnus angustifolia L. & $\mathrm{Ph}$ & FrArb & $\mathrm{He}$ & AlkMsTr & X-HgMs & SMnPrRu & + & + & + & + & Adv, Inv \\
\hline Elaeagnaceae & Hippophae rhamnoides L. & $\mathrm{Ph}$ & $\mathrm{ArbFr}$ & $\mathrm{He}$ & OgMsTr & $\mathrm{Ms}$ & $\mathrm{RuCu}$ & - & + & - & - & Adv \\
\hline Euphorbiaceae & Euphorbia palustris L. & $\mathrm{HKr}$ & Per & $\mathrm{He}$ & $\mathrm{MgTr}$ & $\mathrm{MsHg}$ & $\mathrm{PalPr}$ & + & + & - & - & - \\
\hline Euphorbiaceae & E. sequieriana Nesk. & $\mathrm{HKr}$ & Per & $\mathrm{He}$ & $\mathrm{MsOgTr}$ & MsX & PtStPs & + & + & - & - & - \\
\hline Euphorbiaceae & E semivillosa Prokh & $\mathrm{HKr}$ & Per & $\mathrm{ScHe}$ & MsTr & Ms & PrSMn & + & + & - & - & - \\
\hline Euphorbiaceae & E virogta Wald et Kit. & $\mathrm{HKr}$ & Per & $\mathrm{ScHe}$ & MsTr & Ms & $\mathrm{RuPr}$ & + & + & + & - & - \\
\hline Fabaceae & Amornha fruticosa $\mathrm{L}$. & $\mathrm{nPh}$ & $\mathrm{Er}$ & $\mathrm{ScHe}$ & OgMsTr & $\mathrm{MsX}-\mathrm{Hg}$ & GuRuSil & - & + & + & - & Adv Inv \\
\hline Fabacea & Amorpna rumcosa $\mathrm{I}$ & $\mathrm{HKr}$ & $\begin{array}{l}\text { TI } \\
\text { Per }\end{array}$ & $\mathrm{ScHe}$ & MsTr & XMs & $\mathrm{RuStPr}$ & + & $\begin{array}{l}+ \\
+\end{array}$ & ${ }_{-}^{+}$ & - & av, Inv \\
\hline Fabaceae & A. glycyphyllos L. & $\mathrm{HKr}$ & Per & $\mathrm{HeSc}$ & MgTr & $\begin{array}{l}\text { Avis } \\
\text { Ms }\end{array}$ & Sil & + & - & - & - & - \\
\hline
\end{tabular}




\begin{tabular}{|c|c|c|c|c|c|c|c|c|c|c|c|c|}
\hline Families & Species & $\begin{array}{l}\text { Clima- } \\
\text { morphs }\end{array}$ & $\begin{array}{l}\text { Bio- } \\
\text { morphs }\end{array}$ & $\begin{array}{l}\text { Helio- } \\
\text { morphs }\end{array}$ & $\begin{array}{l}\text { Tropho- } \\
\text { morphs }\end{array}$ & $\begin{array}{l}\text { Hygro- } \\
\text { morphs }\end{array}$ & $\begin{array}{l}\text { Ceno- } \\
\text { morphs }\end{array}$ & $\begin{array}{l}\text { Protec- } \\
\text { ted zone }\end{array}$ & $\begin{array}{c}\text { Recre- } \\
\text { ational } \\
\text { zone }\end{array}$ & $\begin{array}{c}\text { Stationary } \\
\text { recreation } \\
\text { zone }\end{array}$ & $\begin{array}{l}\text { Econo- } \\
\text { mic zone }\end{array}$ & $\begin{array}{c}\text { Rare, ad- } \\
\text { ventive, } \\
\text { types }\end{array}$ \\
\hline Fabaceae & A. varius S. G. Gmel. & $\mathrm{HKr}$ & Per & $\mathrm{He}$ & $\operatorname{OgTr}$ & $\mathrm{MsX}$ & StPtPs & + & + & - & - & - \\
\hline Fabaceae & Caragana arborescens Lam. & $\mathrm{Ph}$ & FrArb & $\mathrm{ScHe}$ & MsTr & MsX & $\mathrm{SilCu}$ & + & - & - & - & Adv \\
\hline Fabaceae & $\begin{array}{l}\text { Chamecytisus ruthenicus (Fisch.ex Wol.) } \\
\text { Klaskov }\end{array}$ & $\mathrm{nPh}$ & $\mathrm{Fr}$ & $\mathrm{ScHe}$ & OgMsTr & MsX & SilPsPtSt & + & - & - & - & - \\
\hline Fabaceae & Genista tinctoria L. & $\mathrm{nPh}$ & $\mathrm{Fr}$ & $\mathrm{ScHe}$ & $\mathrm{MsOgTr}$ & $\mathrm{XMs}$ & PrPsSMn & + & + & - & - & - \\
\hline Fabaceae & Lathyrus pratensis L. & $\mathrm{HKr}$ & Per & $\mathrm{He}$ & $\mathrm{MgTr}$ & Ms & $\mathrm{SMnPr}$ & + & + & - & - & - \\
\hline Fabaceae & L. sylvestris L. & $\mathrm{HKr}$ & Per & $\mathrm{ScHe}$ & MsTr & Ms & SilSMn & + & - & - & - & - \\
\hline Fabaceae & L. tuberosus L. & $\mathrm{G}$ & Per & $\mathrm{He}$ & MsMgTr & $\mathrm{Ms} \mathrm{X}$ & RuPrSt & + & + & - & - & Adv \\
\hline Fabaceae & Lotus ucrainicus Klokov & $\mathrm{HKr}$ & Per & $\mathrm{He}$ & MgMsTr & $\mathrm{XMs}$ & StPr & + & + & - & - & - \\
\hline Fabaceae & Medicago lupulina $\mathrm{L}$. & $\mathrm{HKr}$ & Ann & $\mathrm{He}$ & MsMgTr & Ms & RuSMnPr & + & + & + & - & - \\
\hline Fabaceae & Melilotus albus Medik. & $\mathrm{HKr}$ & Bien & $\mathrm{He}$ & MsTr & MsX & RuPrSt & + & + & + & - & - \\
\hline Fabaceae & M. officinalis (L.) Pall. & $\mathrm{HKr}$ & Bien & $\mathrm{He}$ & $\mathrm{MsTr}$ & $\mathrm{XMs}$ & RuStPr & + & + & + & - & - \\
\hline Fabaceae & Robinia pseudacacia L. & $\mathrm{Ph}$ & Arb & $\mathrm{He}$ & Og-MgTr & $\mathrm{X}-\mathrm{Ms}$ & CuSil & + & + & + & + & Adv \\
\hline Fabaceae & Securigera varia $\mathrm{L}$. & $\mathrm{HKr}$ & Per & $\mathrm{He}$ & $\mathrm{MsTr}$ & XMs & StSMnPr & + & + & - & - & - \\
\hline Fabaceae & Trifolium alpestre $\mathrm{L}$. & $\mathrm{HKr}$ & Per & $\mathrm{He}$ & $\operatorname{MgTr}$ & $\mathrm{XMs}$ & SilStPr & + & + & - & - & - \\
\hline Fabaceae & T. arvense $\mathrm{L}$. & $\mathrm{T}$ & Ann & $\mathrm{He}$ & MsTr & MsX & RuPsSt & + & + & - & - & - \\
\hline Fabaceae & T. medium $\mathrm{L}$. & $\mathrm{G}$ & Per & $\mathrm{ScHe}$ & $\operatorname{MgTr}$ & XMs & SilPr & + & + & + & - & - \\
\hline Fabaceae & T. montanum $\mathrm{L}$. & $\mathrm{HKr}$ & Per & $\mathrm{HeSc}$ & $\operatorname{MgTr}$ & XMs & SilPr & + & + & - & - & - \\
\hline Fabaceae & T. pratense $\mathrm{L}$. & $\mathrm{HKr}$ & Per & $\mathrm{He}$ & $\operatorname{MgTr}$ & HgMs & RuSilPr & + & + & + & - & - \\
\hline Fabaceae & T. repens L. & $\mathrm{HKr}$ & Per & $\mathrm{He}$ & $\operatorname{MgTr}$ & $\mathrm{HgMs}$ & $\mathrm{RuPr}$ & + & + & + & + & - \\
\hline Fabaceae & Vicia angustifolia Reichard & $\mathrm{T}$ & Ann & $\mathrm{He}$ & MsMgTr & $\mathrm{XMs}$ & RuPr & - & + & - & - & Adv \\
\hline Fabaceae & V. cracca $\mathrm{L}$. & $\mathrm{HKr}$ & Per & $\mathrm{He}$ & MsTr & $\mathrm{HgMs}$ & PrSt & + & + & - & - & - \\
\hline Fabaceae & V. hirsuta (L.)S. F. Grag & $\mathrm{T}$ & Ann & $\mathrm{ScHe}$ & $\mathrm{MsTr}$ & MsX & RuSilSt & + & + & - & - & Adv \\
\hline Fabaceae & V. pisiformis L. & $\mathrm{HKr}$ & Per & $\mathrm{He}$ & $\mathrm{MsTr}$ & Ms & Sil & + & - & - & - & - \\
\hline Fabaceae & V. tetrasperma (L.) Schreb. & $\mathrm{T}$ & Ann & $\mathrm{ScHe}$ & $\operatorname{MgTr}$ & $\mathrm{XMs}$ & $\mathrm{RuPr}$ & + & + & - & - & Adv \\
\hline Fagaceae & Quercus robur L. & $\mathrm{Ph}$ & Arb & $\mathrm{ScHe}$ & Og-MgTr & $\begin{array}{l}\text { MsX- } \\
\text { MsHg }\end{array}$ & Sil & + & + & + & + & - \\
\hline Fumariaceae & Corydalis cava (L.) Schweigg. et Korte & $\mathrm{G}$ & Per & $\mathrm{ScHe}$ & $\operatorname{MgTr}$ & Ms & Sil & + & - & - & - & RLD2 \\
\hline Fumariaceae & C. marschalliana Pers. & $\mathrm{G}$ & Per & $\mathrm{ScHe}$ & $\operatorname{MgTr}$ & Ms & Sil & + & - & - & - & RLD2 \\
\hline Fumariaceae & C. solida (L.) Clairv. & $\mathrm{G}$ & Per & $\mathrm{ScHe}$ & $\operatorname{MgTr}$ & Ms & Sil & + & - & - & - & - \\
\hline Fumariaceae & Fumaria schleicheri Soy.-Willem. & $\mathrm{T}$ & Ann & $\mathrm{ScHe}$ & $\operatorname{MsTr}$ & MsX & $\mathrm{Ru}$ & + & + & + & + & Adv \\
\hline Gentianaceae & Centaurium erythraea Rafn. & $\mathrm{HKr}$ & Ann & $\mathrm{ScHe}$ & OgMsTr & $\mathrm{XMs}$ & StPr & + & + & - & - & - \\
\hline Geraniaceae & Erodium cicutarium (L.) L. Her. & $\mathrm{T}$ & Ann & $\mathrm{ScHe}$ & OgMsTr & XMs & RuSilPtSt & + & + & - & - & - \\
\hline Geraniaceae & Geranium collinum Steph. & $\mathrm{HKr}$ & Per & $\mathrm{ScHe}$ & AlkTr & $\mathrm{HgMs}$ & PalHalPr & - & + & - & - & - \\
\hline Geraniaceae & G. divaricatum Ehrh. & $\mathrm{T}$ & Ann & $\mathrm{ScHe}$ & OgMsTr & $\mathrm{XMs}$ & RuSil & + & - & - & - & - \\
\hline Geraniaceae & G. palustre L. & $\mathrm{HKr}$ & Per & $\mathrm{ScHe}$ & $\mathrm{MsTr}$ & $\mathrm{MsHg}$ & PrPal & + & + & - & - & RLD 1 \\
\hline Geraniaceae & G. pratense L. & $\mathrm{HKr}$ & Per & $\mathrm{HeSc}$ & $\mathrm{MsTr}$ & $\mathrm{Ms}$ & PrSMn & + & + & - & - & RLD 4 \\
\hline Geraniaceae & G. pusillum L. & $\mathrm{T}$ & Ann & $\mathrm{He}$ & $\mathrm{MsTr}$ & MsX & $\mathrm{Ru}$ & + & + & + & - & Adv \\
\hline Geraniaceae & G. robertianum $\mathrm{L}$. & $\mathrm{T}$ & Ann & $\mathrm{ScHe}$ & OgMsTr & $\mathrm{XMs}$ & PtSil & + & - & - & - & - \\
\hline Geraniaceae & G. sanguineum L. & $\mathrm{G}$ & Per & $\mathrm{ScHe}$ & $\mathrm{OgTr}$ & $\mathrm{XMs}$ & PsSil & + & - & - & - & - \\
\hline Grossulariaceae & Ribes aureum Pursh & $\mathrm{nPh}$ & Fr & $\mathrm{ScHe}$ & MsTr & Ms & SisMnCu & - & - & + & + & Adv \\
\hline Haloragaceae & Myriophyllum spicatum L. & $\mathrm{HKr}$ & Per & $\mathrm{ScHe}$ & $\mathrm{MsTr}$ & Hyr & $\mathrm{Aq}$ & + & + & + & + & - \\
\hline Juglandaceae & Juglans regia $\mathrm{L}$. & $\mathrm{Ph}$ & Arb & $\mathrm{He}$ & $\operatorname{MsMgTr}$ & Ms & SilCu & - & - & + & + & Adv \\
\hline Lamiaceae & Ajuga genevensis L. & $\mathrm{G}$ & Per & $\mathrm{ScHe}$ & MsTr & $\mathrm{XMs}$ & RuPrSil & + & + & - & - & - \\
\hline Lamiaceae & Ballota nigra $\mathrm{L}$. & $\mathrm{HKr}$ & Per & $\mathrm{ScHe}$ & $\operatorname{MsTr}$ & Ms & $\mathrm{Ru}$ & + & + & + & + & Adv \\
\hline Lamiaceae & Betonica officinalis $\mathrm{L}$. & $\mathrm{HKr}$ & Per & $\mathrm{ScHe}$ & $\operatorname{Og} \operatorname{Tr}$ & Ms & PrSil & + & + & - & - & RLD3 \\
\hline Lamiaceae & Chaiturus marnubiastrum (L.) Reichenb. & $\mathrm{HKr}$ & Ann & $\mathrm{ScHe}$ & $\mathrm{MsTr}$ & $\mathrm{XMs}$ & RuPrSMn & + & - & - & - & - \\
\hline Lamiaceae & Clinopodium vulgare $\mathrm{L}$. & $\mathrm{HKr}$ & Per & $\mathrm{ScHe}$ & $\mathrm{MsTr}$ & $\mathrm{XMs}$ & SilSMn & + & - & - & - & - \\
\hline Lamiaceae & Dracocephalum thymiflorum $\mathrm{L}$. & $\mathrm{HKr}$ & Ann & $\mathrm{ScHe}$ & OgMsTr & $\mathrm{MsX}$ & StSilRu & + & - & - & - & Adv \\
\hline Lamiaceae & Galeopsis tetrahit L. & $\mathrm{T}$ & Ann & $\mathrm{ScHe}$ & OgMsTr & Ms & RuPsSil & + & + & - & - & - \\
\hline Lamiaceae & Glechoma hederacea L. & $\mathrm{HKr}$ & Per & $\mathrm{HeSc}$ & $\mathrm{MsTr}$ & Ms & RuPrSil & + & + & - & - & - \\
\hline Lamiaceae & Lamium amplexicaule $\mathrm{L}$. & $\mathrm{HKr}$ & Ann & $\mathrm{He}$ & OgMsTr & $\mathrm{XMs}$ & $\mathrm{Ru}$ & + & + & + & - & Adv \\
\hline Lamiaceae & L. purpureum L. & $\mathrm{HKr}$ & Ann & $\mathrm{ScHe}$ & MsTr & $\mathrm{XMs}$ & $\mathrm{Ru}$ & - & + & + & - & Adv \\
\hline Lamiaceae & Leonurus cardiaca L. & $\mathrm{HKr}$ & Per & $\mathrm{ScHe}$ & $\operatorname{MgTr}$ & $\mathrm{XMs}$ & SMnRu & + & + & - & - & Adv \\
\hline Lamiaceae & L. villosus Desf. ex D’Urv. & $\mathrm{HKr}$ & Per & $\mathrm{ScHe}$ & MsTr & MsX & $\mathrm{Ru}$ & + & + & + & - & - \\
\hline Lamiaceae & Lycopus europaeus L. & $\mathrm{HKr}$ & Per & $\mathrm{ScHe}$ & $\mathrm{MgTr}$ & $\mathrm{MsHg}$ & PrPal & + & + & + & + & - \\
\hline Lamiaceae & L. exaltatus L. fil. & $\mathrm{HKr}$ & Per & $\mathrm{ScHe}$ & $\mathrm{MgTr}$ & $\mathrm{MsHg}$ & PrPal & + & + & - & - & - \\
\hline Lamiaceae & Mentha arvensis L. & $\mathrm{HKr}$ & Per & $\mathrm{ScHe}$ & MsTr & $\mathrm{HgMs}$ & RuPrSil & + & + & - & - & - \\
\hline Lamiaceae & M. aquatica L. & $\mathrm{HKr}$ & Per & $\mathrm{ScHe}$ & MsTr & $\mathrm{Hg}$ & PalAq & + & + & + & - & - \\
\hline Lamiaceae & Nepeta cataria $\mathrm{L}$. & $\mathrm{HKr}$ & Per & $\mathrm{ScHe}$ & $\mathrm{MsTr}$ & $\mathrm{XMs}$ & RuStSil & + & + & - & - & Adv \\
\hline Lamiaceae & Origanum vulgare L. & $\mathrm{HKr}$ & Per & $\mathrm{ScHe}$ & $\operatorname{MgTr}$ & MsX & StSMnPr & + & - & - & - & - \\
\hline Lamiaceae & Prunella vulgaris L. & $\mathrm{HKr}$ & Per & $\mathrm{He}$ & $\operatorname{MgTr}$ & Ms & RuPrSil & + & - & - & - & - \\
\hline Lamiaceae & Salvia nemorosa $\mathrm{L}$. & $\mathrm{HKr}$ & Per & $\mathrm{He}$ & $\mathrm{MsTr}$ & $\mathrm{Ms} \mathrm{X}$ & SMnPrSt & + & + & - & - & - \\
\hline Lamiaceae & S. verticillata $\mathrm{L}$. & $\mathrm{HKr}$ & Per & $\mathrm{ScHe}$ & $\mathrm{MsTr}$ & $\mathrm{MsX}$ & PrStRu & + & + & - & - & - \\
\hline Lamiaceae & Scutellaria galericulata L. & $\mathrm{G}$ & Per & $\mathrm{ScHe}$ & $\operatorname{MgTr}$ & $\mathrm{Hg}$ & PrPal & + & + & - & - & - \\
\hline Lamiaceae & S. hastifolia L. & $\mathrm{HKr}$ & Per & $\mathrm{ScHe}$ & $\operatorname{MgTr}$ & HgMs & PalPr & + & - & - & - & - \\
\hline Lamiaceae & Stachys palustris L. & G & Per & $\mathrm{ScHe}$ & $\operatorname{MgTr}$ & $\mathrm{Hg}$ & PrPal & + & + & - & - & - \\
\hline Lamiaceae & S. sylvatica L. & $\mathrm{HKr}$ & Per & $\mathrm{HeSc}$ & $\operatorname{MgTr}$ & Ms & Sil & + & - & - & - & - \\
\hline Lamiaceae & Teucrium scordium $\mathrm{L}$. & $\mathrm{HKr}$ & Per & $\mathrm{ScHe}$ & $\operatorname{MgTr}$ & $\mathrm{HgMs}$ & PalPr & + & + & - & - & - \\
\hline Lamiaceae & Thymus pallasianus Heinr. Braun & $\mathrm{Ch}$ & Per & $\mathrm{ScHe}$ & $\mathrm{Og} \operatorname{Tr}$ & MsX & SMnPs & + & + & - & - & - \\
\hline Lentibulariaceae & Utricularia vulgaris $\mathrm{L}$. & $\mathrm{HKr}$ & Per & $\mathrm{ScHe}$ & $\operatorname{MgTr}$ & Hy er & $\mathrm{Aq}$ & + & - & - & - & RLD3 \\
\hline Loranthaceae & Viscum album L. & $\mathrm{nPh}$ & $\mathrm{Fr}$ & $\mathrm{ScHe}$ & Par & $\mathrm{HgMs}$ & Sil & + & + & - & - & - \\
\hline Lythraceae & Lythrum salicaria L. & $\mathrm{HKr}$ & Per & $\mathrm{He}$ & $\operatorname{MgTr}$ & $\mathrm{MsHg}$ & PrAqPal & + & + & - & - & - \\
\hline Lythraceae & L. virgatum $\mathrm{L}$. & $\mathrm{HKr}$ & Per & $\mathrm{He}$ & $\operatorname{MgTr}$ & $\mathrm{MsHg}$ & AqPalPr & + & + & - & - & - \\
\hline Lythraceae & Peplis portula L. & $\mathrm{HKr}$ & Ann & $\mathrm{ScHe}$ & MsTr & $\mathrm{MsHg}$ & PsPalPr & + & + & - & - & - \\
\hline Malvaceae & Althaea officinalis L. & $\mathrm{HKr}$ & Per & $\mathrm{He}$ & AlkMgTr & Ms & HalPalPr & + & + & - & - & Adv \\
\hline Malvaceae & Lavatera thuringiaca $\mathrm{L}$. & $\mathrm{HKr}$ & Per & $\mathrm{ScHe}$ & $\operatorname{MgTr}$ & MsX & RuStPr & + & + & - & - & - \\
\hline Malvaceae & Malva pusilla Smith & $\mathrm{T}$ & Ann & $\mathrm{ScHe}$ & MsTr & $\mathrm{XMs}$ & $\mathrm{Ru}$ & + & + & + & + & Adv \\
\hline Moraceae & Morus alba $\mathrm{L}$. & $\mathrm{Ph}$ & Arb & $\mathrm{He}$ & $\mathrm{MsTr}$ & Ms & CuSilRu & + & + & + & + & Adv \\
\hline Nymphaeaceae & Nuphar lutea (L.) Smith & $\mathrm{HKr}$ & Per & $\mathrm{He}$ & MsTr & $\mathrm{Plr}$ & $\mathrm{Aq}$ & + & + & - & - & RLD 3 \\
\hline
\end{tabular}




\begin{tabular}{|c|c|c|c|c|c|c|c|c|c|c|c|c|}
\hline Families & Species & $\begin{array}{l}\text { Clima- } \\
\text { morphs }\end{array}$ & $\begin{array}{l}\text { Bio- } \\
\text { morphs }\end{array}$ & $\begin{array}{l}\text { Helio- } \\
\text { morphs }\end{array}$ & $\begin{array}{l}\text { Tropho- } \\
\text { morphs }\end{array}$ & $\begin{array}{l}\text { Hygro- } \\
\text { morphs }\end{array}$ & $\begin{array}{l}\text { Ceno- } \\
\text { morphs }\end{array}$ & $\begin{array}{l}\text { Protec- } \\
\text { ted zone }\end{array}$ & $\begin{array}{c}\text { Recre- } \\
\text { ational } \\
\text { zone }\end{array}$ & $\begin{array}{c}\text { Stationary } \\
\text { recreation } \\
\text { zone }\end{array}$ & $\begin{array}{l}\text { Econo- } \\
\text { mic zone }\end{array}$ & $\begin{array}{c}\text { Rare, ad- } \\
\text { ventive, } \\
\text { types }\end{array}$ \\
\hline Nymphaeaceae & Nymphaea alba L. & $\mathrm{HKr}$ & Per & $\mathrm{ScHe}$ & MsTr & $\mathrm{Plr}$ & $\mathrm{Aq}$ & + & + & - & - & RLD2 \\
\hline Oleaceae & Fraxinus excelsior $\mathrm{L}$. & $\mathrm{Ph}$ & Arb & $\mathrm{ScHe}$ & MsMgTr & $\begin{array}{l}\text { MsX- } \\
\text { MsHg }\end{array}$ & Sil & + & + & + & + & - \\
\hline Oleaceae & Ligustrum vulgare L. & $\mathrm{Ph}$ & $\mathrm{Fr}$ & $\mathrm{ScHe}$ & MsTr & MsX-Ms & PtSil & - & + & + & + & Adv \\
\hline Oleaceae & Syringa vulgaris L. & $\mathrm{nPh}$ & $\mathrm{Fr}$ & $\mathrm{ScHe}$ & $\operatorname{MsTr}$ & MsX & $\mathrm{Cu}$ & - & - & - & + & Adv \\
\hline Onagraceae & Epilobium hirsutum L. & $\mathrm{HKr}$ & Per & $\mathrm{He}$ & $\operatorname{MgTr}$ & $\mathrm{MsHg}$ & PrPal & + & + & - & - & - \\
\hline Onagraceae & E. roseum Schreb. & $\mathrm{HKr}$ & Per & $\mathrm{ScHe}$ & $\operatorname{MgTr}$ & $\mathrm{HgMs}$ & $\mathrm{PalPr}$ & + & - & - & - & - \\
\hline Onagraceae & Oenothera biennis L. & $\mathrm{HKr}$ & Bien & $\mathrm{ScHe}$ & OgMsTr & $\mathrm{XMs}$ & PsRu & + & + & + & - & Adv \\
\hline Orobanchaceae & Orobanche cumana Wallr. & $\mathrm{TG}$ & Per & $\mathrm{He}$ & Par & XMs & $\mathrm{Ru}$ & + & + & - & - & Adv \\
\hline Orobanchaceae & Pheliplanche arenaria (Borkh.) Walp. & G & Per & $\mathrm{He}$ & Par & MsX & RuPs & + & + & - & - & Adv \\
\hline Papaveraceae & Chelidonium majus L. & $\mathrm{HKr}$ & Per & $\mathrm{HeSc}$ & MsMgTr & Ms & RuSil & + & + & + & + & - \\
\hline Plantaginaceae & Plantago arenaria Waldst. et Kit. & $\mathrm{T}$ & Ann & $\mathrm{He}$ & $\mathrm{Og} \operatorname{Tr}$ & MsX & RuPs & + & + & + & - & - \\
\hline Plantaginaceae & P. major $\mathrm{L}$ & $\mathrm{HKr}$ & Per & $\mathrm{He}$ & $\operatorname{MgTr}$ & Ms & RuPr & + & + & + & + & - \\
\hline Plantaginaceae & P. media $\mathrm{L}$. & $\mathrm{HKr}$ & Per & $\mathrm{He}$ & $\operatorname{MgTr}$ & MsX & RuSiPrSt & + & + & - & - & - \\
\hline Plantaginaceae & P. scabra Moench. & $\mathrm{T}$ & Ann & $\mathrm{He}$ & OgTr & MsXX & RuPs & + & + & + & - & - \\
\hline Polygalaceae & Polygala comosa Schruhr & $\mathrm{HKr}$ & Per & $\mathrm{ScHe}$ & MsTr & Ms & PrSMn & + & + & - & - & - \\
\hline Polygonaceae & Fallopia convolvulus (L.) A. Love & $\mathrm{T}$ & Ann & $\mathrm{ScHe}$ & $\operatorname{MsTr}$ & XMs & $\mathrm{Ru}$ & + & + & - & - & Adv \\
\hline Polygonaceae & Persicaria amphybia (L.) Delarbre & $\mathrm{HKr}$ & Per & $\mathrm{ScHe}$ & $\mathrm{MsTr}$ & $\mathrm{PlrHg}$ & AqPal & + & + & - & - & - \\
\hline Polygonaceae & P. hydropiper $\mathrm{L}$. & $\mathrm{T}$ & Ann & $\mathrm{ScHe}$ & MsTr & $\mathrm{HgMs}$ & RuSiPalPr & + & + & - & - & - \\
\hline Polygonaceae & P. lapathifolia (L.) Delarbe & $\mathrm{T}$ & Ann & $\mathrm{He}$ & OgTr & HgMs & PalPsPr & + & + & - & - & - \\
\hline Polygonaceae & $\begin{array}{l}\text { P. lapathifolia (L.) Delarbe subsp. andrze- } \\
\text { jowskiana (Klokov) Sojak }\end{array}$ & $\mathrm{T}$ & Ann & $\mathrm{He}$ & OgAlkTr & $\mathrm{HgMs}$ & PalPr & + & + & - & - & - \\
\hline Polygonaceae & P. maculosa S.F. Gray & $\mathrm{T}$ & Ann & $\mathrm{He}$ & $\operatorname{MsTr}$ & Ms & $\operatorname{RuPr}$ & + & + & - & - & - \\
\hline Polygonaceae & Polygonum arenarium Waldst. et Kit. & $\mathrm{T}$ & Ann & $\mathrm{He}$ & OgTr & Ms & SilPs & + & + & + & - & - \\
\hline Polygonaceae & P. aviculare L. & $\mathrm{T}$ & Ann & $\mathrm{ScHe}$ & MsTr & MsX & $\mathrm{Ru}$ & + & + & + & + & - \\
\hline Polygonaceae & Rumex acetos $a \mathrm{~L}$. & G & Per & $\mathrm{He}$ & $\operatorname{MgTr}$ & XMs & $\mathrm{SMnPr}$ & + & - & - & - & - \\
\hline Polygonaceae & R.acetosella L. & $\mathrm{HKr}$ & Per & $\mathrm{ScHe}$ & OgMsTr & Ms & PrSMnPs & - & + & + & - & - \\
\hline Polygonaceae & R.confertus Willd. & $\mathrm{HKr}$ & Per & $\mathrm{ScHe}$ & $\mathrm{MsTr}$ & XMs & RuPrSMn & + & + & + & + & - \\
\hline Polygonaceae & R.crispus L. & $\mathrm{HKr}$ & Per & $\mathrm{He}$ & MsTr & Ms & RuPr & + & + & + & + & - \\
\hline Polygonaceae & R. hydrolapatum Huds. & $\mathrm{HKr}$ & Per & $\mathrm{He}$ & $\mathrm{MsTr}$ & $\mathrm{Hg}$ & AqPal & + & + & - & - & - \\
\hline Portulacaceae & Portulaca oleracea L. & $\mathrm{T}$ & Ann & $\mathrm{ScHe}$ & $\operatorname{MsTr}$ & $\mathrm{XMs}$ & $\mathrm{Ru}$ & - & + & + & + & Adv \\
\hline Primulaceae & Lysimachia nummularia $\mathrm{L}$. & $\mathrm{HKr}$ & Per & $\mathrm{ScHe}$ & $\operatorname{MgTr}$ & $\mathrm{HgMs}$ & SilPr & + & + & - & - & - \\
\hline Primulaceae & L. vulgaris $\mathrm{L}$. & $\mathrm{HKr}$ & Per & $\mathrm{ScHe}$ & $\operatorname{MgTr}$ & $\mathrm{MsHg}$ & $\mathrm{Pal}$ & + & + & - & - & - \\
\hline Ranunculaceae & Anemone ranunculoides (L.) Holub & $\mathrm{G}$ & Per & $\mathrm{HeSc}$ & $\operatorname{MgTr}$ & Ms & Sil & + & - & - & - & RLD3 \\
\hline Ranunculaceae & Batrachium circinatum (Sibth.) Spach & $\mathrm{HKr}$ & Per & $\mathrm{HeSc}$ & $\operatorname{MgTr}$ & Hyr & $\mathrm{Aq}$ & + & + & + & + & - \\
\hline Ranunculaceae & B. trichophyllum (Chaix) Bosch & $\mathrm{HKr}$ & Per & $\mathrm{HeSc}$ & $\operatorname{MgTr}$ & Hy $r$ & $\mathrm{Aq}$ & - & + & - & - & - \\
\hline Ranunculaceae & Ficaria verna Huds. & $\mathrm{G}$ & Per & $\mathrm{HeSc}$ & $\operatorname{MgTr}$ & Ms & Sil & + & + & + & + & - \\
\hline Ranunculaceae & Ranunculus illiricus $\mathrm{L}$. & G & Per & $\mathrm{ScHe}$ & MsMgTr & $\mathrm{XMs}$ & PrSt & + & + & - & - & - \\
\hline Ranunculaceae & R. lingua $\mathrm{L}$. & $\mathrm{HKr}$ & Per & $\mathrm{ScHe}$ & MsTr & $\mathrm{MsHg}$ & PrPal & + & - & - & - & RLD3 \\
\hline Ranunculaceae & R. polyanthemos L. & $\mathrm{HKr}$ & Per & $\mathrm{ScHe}$ & MsMgTr & $\mathrm{XMs}$ & StSMnPr & + & + & - & - & - \\
\hline Ranunculaceae & R. repens $\mathrm{L}$. & $\mathrm{HKr}$ & Per & $\mathrm{ScHe}$ & $\operatorname{MgTr}$ & $\mathrm{HgMs}$ & PalPr & + & + & + & - & - \\
\hline Ranunculaceae & R. sceleratus L. & $\mathrm{T}$ & Ann & $\mathrm{ScHe}$ & $\operatorname{MsMgTr}$ & $\mathrm{HgMs}$ & PrPal & + & + & + & - & - \\
\hline Ranunculaceae & Thalictrum flavum $\mathrm{L}$. & $\mathrm{HKr}$ & Per & $\mathrm{ScHe}$ & $\operatorname{MsMgTr}$ & $\mathrm{Hg}$ & PalPr & + & + & - & - & - \\
\hline Rhamnacaceae & Frangula alnus Mill. & $\mathrm{nPh}$ & Per & $\mathrm{HeSc}$ & Og-MgTr & Ms-Hg & SMnSil & + & - & - & - & - \\
\hline Rhamnacaceae & Rhammus catartica $\mathrm{L}$. & $\mathrm{nPh}$ & Per & $\mathrm{ScHe}$ & MgMsTr & $\mathrm{XMs}$ & SilSMn & + & - & - & - & - \\
\hline Rosaceae & Agrimonia eupatoria $\mathrm{L}$. & $\mathrm{HKr}$ & Per & $\mathrm{ScHe}$ & MgMsTr & $\mathrm{XMs}$ & SMnSt & + & + & + & - & - \\
\hline Rosaceae & Armeniaca vulgaris Lam. & $\mathrm{Ph}$ & Arb & $\mathrm{He}$ & OgMsTr & MsX & RuSilCu & - & + & + & + & Adv \\
\hline Rosaceae & Cerasus avium (L.) Moench & $\mathrm{Ph}$ & Arb & $\mathrm{ScHe}$ & MsMgTr & Ms & $\mathrm{SilCu}$ & - & - & + & + & - \\
\hline Rosaceae & C.mahaleb (L.) Mill. & $\mathrm{Ph}$ & ArbFr & $\mathrm{ScHe}$ & MsTr & $\mathrm{XMs}$ & CuSMn & - & + & + & + & Adv \\
\hline Rosaceae & C.vulgaris Mill. & $\mathrm{Ph}$ & Arb & $\mathrm{ScHe}$ & $\operatorname{MgTr}$ & $\mathrm{XMs}$ & $\mathrm{RuCu}$ & - & - & + & + & Adv \\
\hline Rosaceae & Crataegus fallacina Klokov & $\mathrm{Ph}$ & $\mathrm{Fr}$ & $\mathrm{ScHe}$ & MsTr & MsX & SMnSil & + & + & - & - & - \\
\hline Rosaceae & C. leiomonogyna Klokov & $\mathrm{Ph}$ & ArbFr & $\mathrm{ScHe}$ & MsTr & MsX & SMnPtSt & & & & & \\
\hline Rosaceae & Filipendula ulmaria (L.) Maxim. & G & Per & $\mathrm{HeSc}$ & $\operatorname{MgTr}$ & $\mathrm{MsHg}$ & $\mathrm{SMnPalPr}$ & + & - & - & - & - \\
\hline Rosaceae & Fragaria viridis Duch. & $\mathrm{HKr}$ & Per & $\mathrm{ScHe}$ & MsTr & $\mathrm{XMs}$ & StSMn & + & - & - & - & - \\
\hline Rosaceae & Geum urbanum L. & $\mathrm{HKr}$ & Per & $\mathrm{ScHe}$ & OgMsTr & Ms & RuSil & + & + & + & + & - \\
\hline Rosaceae & Malus domestica Borkh. & $\mathrm{Ph}$ & Arb & $\mathrm{ScHe}$ & MsTr & Ms & RuCul & - & - & + & + & Adv \\
\hline Rosaceae & Potentilla anserina $\mathrm{L}$. & $\mathrm{HKr}$ & Per & $\mathrm{He}$ & AlkMgTr & $\mathrm{MsHg}$ & $\operatorname{Pr}$ & + & + & + & - & - \\
\hline Rosaceae & P. argentea $\mathrm{L}$. & $\mathrm{HKr}$ & Per & $\mathrm{He}$ & MsTr & MsX & SilPrStRu & + & + & + & + & - \\
\hline Rosaceae & P. incana P. Gaertn., B. Mey. \& Scherb. & $\mathrm{HKr}$ & Per & $\mathrm{ScHe}$ & OgMsTr & XMs & PtStPs & + & - & - & - & - \\
\hline Rosaceae & P. reptans $\mathrm{L}$. & $\mathrm{HKr}$ & Per & $\mathrm{He}$ & MsTr & $\mathrm{HgMs}$ & $\mathrm{SMnPr}$ & + & + & - & - & - \\
\hline Rosaceae & P. supina $\mathrm{L}$. & $\mathrm{HKr}$ & Ann & $\mathrm{He}$ & MsMgTr & Ms & $\operatorname{PrRu}$ & + & + & - & - & - \\
\hline Rosaceae & Prumus domestica $\mathrm{L}$. & $\mathrm{Ph}$ & $\mathrm{Fr}$ & $\mathrm{ScHe}$ & MsTr & MsX & $\mathrm{SMnCu}$ & - & - & - & + & Adv \\
\hline Rosaceae & P. stepposa Kotov & $\mathrm{Ph}$ & $\mathrm{Fr}$ & $\mathrm{ScHe}$ & MsTr & MsX & SMnSt & + & + & + & + & - \\
\hline Rosaceae & Pyrus communis L. & $\mathrm{Ph}$ & Arb & $\mathrm{ScHe}$ & MgMsTr & MsX & StSMnSil & + & + & + & + & - \\
\hline Rosaceae & Rosa canina $\mathrm{L}$. & $\mathrm{nPh}$ & $\mathrm{Fr}$ & $\mathrm{ScHe}$ & MsTr & $\mathrm{X}-\mathrm{Ms}$ & RuSMnSt & + & + & + & + & - \\
\hline Rosaceae & Rubus caesius L. & $\mathrm{nPh}$ & $\mathrm{Fr}$ & $\mathrm{ScHe}$ & MsT & Ms & RuSil & + & + & + & - & - \\
\hline Rubiaceae & Galium aparine $\mathrm{L}$. & $\mathrm{T}$ & Ann & $\mathrm{ScHe}$ & $\operatorname{MgTr}$ & XMs & SilRu & + & + & + & - & - \\
\hline Rubiaceae & G. mollugo L. & $\mathrm{HKr}$ & Per & $\mathrm{ScHe}$ & MsTr & XMs & SilPr & + & + & + & - & - \\
\hline Rubiaceae & G. palustre L. & $\mathrm{HKr}$ & Per & $\mathrm{ScHe}$ & $\operatorname{MgTr}$ & $\mathrm{MsHg}$ & PalPr & + & + & - & - & - \\
\hline Rubiaceae & G. physocarpum Ledeb. & $\mathrm{HKr}$ & Per & $\mathrm{HeSc}$ & $\operatorname{MgTr}$ & Ms & SilPr & + & + & - & - & - \\
\hline Rubiaceae & G. rivale (Sibth.et Smim) Griseb. & $\mathrm{HKr}$ & Per & $\mathrm{HeSc}$ & $\mathrm{MsTr}$ & $\mathrm{HgMs}$ & $\mathrm{SMnPr}$ & + & - & - & - & - \\
\hline Rubiaceae & G. rubioides L. & $\mathrm{HKr}$ & Per & $\mathrm{He}$ & $\operatorname{MsTr}$ & Ms & $\operatorname{Pr}$ & + & + & - & - & - \\
\hline Rubiaceae & G. uliginosum L. & $\mathrm{HKr}$ & Per & $\mathrm{HeSc}$ & MsTr & $\mathrm{MsHg}$ & PrSilPal & + & + & - & - & RLD 4 \\
\hline Rubiaceae & G. verum $\mathrm{L}$. & $\mathrm{HKr}$ & Per & $\mathrm{ScHe}$ & $\mathrm{MsTr}$ & $\mathrm{XMs}$ & PsSilSt & + & + & - & - & - \\
\hline Salicaceae & Populus alba L. & $\mathrm{Ph}$ & Arb & $\mathrm{He}$ & OgMsTr & $\mathrm{XMs-Hg}$ & Sil & + & + & + & + & - \\
\hline Salicaceae & P.nigra L. & $\mathrm{Ph}$ & Arb & $\mathrm{He}$ & OgMsTr & $\mathrm{XMs}-\mathrm{Hg}$ & Sil & + & + & + & + & - \\
\hline Salicaceae & P. tremula L. & $\mathrm{Ph}$ & Arb & $\mathrm{ScHe}$ & OgMsTr & $\mathrm{HgMs}$ & Sil & + & + & - & - & - \\
\hline Salicaceae & Salix acutifolia Willd. & $\mathrm{Ph}$ & $\mathrm{Fr}$ & $\mathrm{ScHe}$ & OgTr & $\mathrm{HgMs}$ & SisMnPs & + & + & + & - & - \\
\hline Salicaceae & S. alba L. & $\mathrm{Ph}$ & Arb & $\mathrm{ScHe}$ & Og-MgTr & XMs-Hg & Sil & + & + & + & + & - \\
\hline Salicaceae & S. cinerea $\mathrm{L}$. & $\mathrm{Ph}$ & $\mathrm{Fr}$ & $\mathrm{ScHe}$ & MgMsTr & $\mathrm{MsHg}$ & SilPal & + & - & - & - & - \\
\hline
\end{tabular}




\begin{tabular}{|c|c|c|c|c|c|c|c|c|c|c|c|c|}
\hline Families & Species & $\begin{array}{l}\text { Clima- } \\
\text { morphs }\end{array}$ & $\begin{array}{c}\text { Bio- } \\
\text { morphs }\end{array}$ & $\begin{array}{l}\text { Helio- } \\
\text { morphs }\end{array}$ & $\begin{array}{l}\text { Tropho- } \\
\text { morphs }\end{array}$ & $\begin{array}{l}\text { Hygro- } \\
\text { morphs }\end{array}$ & $\begin{array}{l}\text { Ceno- } \\
\text { morphs }\end{array}$ & $\begin{array}{l}\text { Protec- } \\
\text { ted zone }\end{array}$ & $\begin{array}{l}\text { Recre- } \\
\text { ational } \\
\text { zone }\end{array}$ & $\begin{array}{l}\text { Stationary } \\
\text { recreation } \\
\text { zone }\end{array}$ & $\begin{array}{l}\text { Econo- } \\
\text { mic zone }\end{array}$ & $\begin{array}{l}\text { Rare, ad- } \\
\text { ventive, } \\
\text { types }\end{array}$ \\
\hline Salicaceae & S. fragilis $\mathrm{L}$. & $\mathrm{Ph}$ & Arb & $\mathrm{He}$ & MsTr & $\begin{array}{l}\text { XMs- } \\
\text { MsHg }\end{array}$ & SilPr & + & + & + & + & Adv \\
\hline Salicaceae & S. pentandra $\mathrm{L}$. & $\mathrm{Ph}$ & $\mathrm{Fr}$ & $\mathrm{HeSc}$ & $\operatorname{Ms} T r$ & $\mathrm{MsHg}$ & PalSil & + & + & - & - & - \\
\hline Salicaceae & S. triandra L. & $\mathrm{Ph}$ & Fr & $\mathrm{He}$ & $\operatorname{MgTr}$ & Ms-Hg & SilPr & + & + & + & + & - \\
\hline Salicaceae & S. vinogradovii A. Skvorts. & $\mathrm{Ph}$ & $\mathrm{Fr}$ & $\mathrm{He}$ & OgMsTr & $\mathrm{HgMs}$ & SilPr & + & + & - & - & - \\
\hline Scrophulariaceae & Euphrasia pectinata Ten. & $\mathrm{T}$ & Ann & $\mathrm{ScHe}$ & $\mathrm{MsTr}$ & $X$ & SilSt & + & + & + & + & - \\
\hline Scrophulariaceae & Gratiola officinalis L. & $\mathrm{HKr}$ & Per & $\mathrm{ScHe}$ & Og-MgTr & Ms & PsPr & + & + & - & - & - \\
\hline Scrophulariaceae & Linaria dulcis Klok. & $\mathrm{G}$ & Per & $\mathrm{He}$ & OgTr & MsX & SilPs & + & + & - & - & - \\
\hline Scrophulariaceae & L. vulgaris Mill. & G & Per & $\mathrm{ScHe}$ & $\mathrm{MsTr}$ & MsX & SMnPrRu & + & + & + & - & - \\
\hline Scrophulariaceae & Melampyrum cristatum L. & $\mathrm{T}$ & Ann & $\mathrm{ScHe}$ & $\mathrm{MsTr}$ & OgMs & SilPsPr & + & + & - & - & RLD3 \\
\hline Scrophulariaceae & M. nemorosum L. & $\mathrm{T}$ & Ann & $\mathrm{ScHe}$ & OgMsTr & Ms & PsPrSil & + & - & - & - & RLD3 \\
\hline Scrophulariaceae & Odontines vulgaris Moench & $\mathrm{T}$ & Ann & $\mathrm{ScHe}$ & MsTr & MsX & RuStPr & + & + & + & - & - \\
\hline Scrophulariaceae & Rhinanthus vermalis (N.Zing.) Schischk.et Serg. & $\mathrm{T}$ & Ann & $\mathrm{He}$ & $\mathrm{Ms} T r$ & Ms & RuSMnPr & + & + & - & - & - \\
\hline Scrophulariaceae & Scrophularia nodosa L. & $\mathrm{HKr}$ & Per & $\mathrm{HeSc}$ & MsMgTr & Ms & PrSMnSil & + & + & - & - & - \\
\hline Scrophulariaceae & Verbascum densiflonum Bertol. & $\mathrm{HKr}$ & Bien & $\mathrm{He}$ & MsMgTr & $\mathrm{XMs}$ & RuPsSMn & + & + & - & - & - \\
\hline Scrophulariaceae & V. lychnitis L. & $\mathrm{HKr}$ & Bien & $\mathrm{ScHe}$ & OgMsTr & MsX & RuSMn & + & + & - & - & - \\
\hline Scrophulariaceae & Veronica anagallis-aquatica L. & $\mathrm{HKr}$ & Per & $\mathrm{He}$ & MsTr & HelHg & AqPal & + & - & - & - & - \\
\hline Scrophulariaceae & $V$. arvensis $\mathrm{L}$. & $\mathrm{HKr}$ & Ann & $\mathrm{He}$ & $\mathrm{MsTr}$ & MsX & $\mathrm{StRu}$ & + & + & - & - & Adv \\
\hline Scrophulariaceae & V. chamaedrys $\mathrm{L}$. & $\mathrm{HKr}$ & Per & $\mathrm{ScHe}$ & $\mathrm{MsTr}$ & Ms & PrSMn & + & + & - & - & - \\
\hline Scrophulariaceae & V. dillenii Crantz & $\mathrm{HKr}$ & Bien & $\mathrm{ScHe}$ & $\mathrm{OgTr}$ & MsX & Sil & + & + & + & - & - \\
\hline Scrophulariaceae & V. longifolia L. & $\mathrm{HKr}$ & Per & $\mathrm{ScHe}$ & $\operatorname{MgTr}$ & $\mathrm{HgMs}$ & SilPr & + & - & - & - & - \\
\hline Scrophulariaceae & V.persica Poir. & $\mathrm{T}$ & Ann & $\mathrm{He}$ & $\mathrm{MsTr}$ & XMs & $\mathrm{Ru}$ & - & + & - & - & Adv \\
\hline Scrophulariaceae & V. scutellata $\mathrm{L}$. & $\mathrm{HKr}$ & Per & $\mathrm{He}$ & Og-MgTr & $\mathrm{MsHg}$ & PalPr & + & - & - & - & RLD 2 \\
\hline Scrophulariaceae & V. spuria $\mathrm{L}$. & $\mathrm{HKr}$ & Per & $\mathrm{ScHe}$ & Og-MgTr & $\mathrm{XMs}$ & StSMn & + & + & - & - & - \\
\hline Scrophulariaceae & V. teucrium $\mathrm{L}$. & $\mathrm{HKr}$ & Per & $\mathrm{ScHe}$ & OgMsTr & $\mathrm{XMs}$ & StSMn & + & + & - & - & - \\
\hline Scrophulariaceae & V. verna $\mathrm{L}$. & $\mathrm{HKr}$ & Bien & $\mathrm{He}$ & MsTr & MsX & $\mathrm{PrStRu}$ & + & + & - & - & - \\
\hline Simarubaceae & Ailanthus altissima (Mill.) Swingle & $\mathrm{Ph}$ & Arb & $\mathrm{ScHe}$ & OgMsTr & X-Ms & SilCuRu & - & - & - & + & Adv, Inv \\
\hline Solanaceae & Datura stramonium L. & $\mathrm{T}$ & Ann & $\mathrm{He}$ & $\operatorname{MgTr}$ & Ms & $\mathrm{Ru}$ & - & - & + & + & $\mathrm{Adv}$ \\
\hline Solanaceae & Hyoscyamus niger L. & $\mathrm{HKr}$ & Bien & $\mathrm{He}$ & $\mathrm{Ms} \operatorname{Tr}$ & MsX & $\mathrm{Ru}$ & - & - & + & + & Adv \\
\hline Solanaceae & Lycium barbatum L. & $\mathrm{nPh}$ & $\mathrm{Fr}$ & $\mathrm{He}$ & OgMsTr & MsX & $\mathrm{CuRu}$ & - & - & - & + & Adv \\
\hline Solanaceae & Lycopersicon esculenthum Mill. & $\mathrm{T}$ & Ann & $\mathrm{He}$ & $\operatorname{MgTr}$ & Ms & $\mathrm{RuCu}$ & - & + & + & + & Adv \\
\hline Solanaceae & Solanum dulcamara $\mathrm{L}$ & $\mathrm{Ch}$ & Per & $\mathrm{ScHe}$ & OgMsTr & $\mathrm{MsHg}$ & SilPal & + & + & - & - & - \\
\hline Solanaceae & S. nigrum L. & $\mathrm{T}$ & Ann & $\mathrm{He}$ & MsTr & $\mathrm{Ms}$ & $\mathrm{Ru}$ & - & + & + & + & Adv \\
\hline Tiliaceae & Tilia cordata Mill. & $\mathrm{Ph}$ & Arb & $\mathrm{ScHe}$ & MsMgTr & Ms & Sil & + & - & - & - & - \\
\hline Ulmaceae & Ulmus laevis Pall. & $\mathrm{Ph}$ & Arb & $\mathrm{HeSc}$ & Og-MgTr & $\begin{array}{l}\text { XMs- } \\
\text { MsHg }\end{array}$ & Sil & + & + & - & - & - \\
\hline Ulmaceae & U. minor Mill. & $\mathrm{Ph}$ & Arb & $\mathrm{ScHe}$ & $\mathrm{Ms} \operatorname{Tr}$ & MsX & SilSMn & + & + & - & - & - \\
\hline Ulmaceae & U. pumila L. & $\mathrm{Ph}$ & Arb & $\mathrm{ScHe}$ & OgMsTr & MsX & $\mathrm{SilCuRu}$ & + & + & + & + & Adv, Inv \\
\hline Ulmaceae & U. suberosa Moench & $\mathrm{Ph}$ & Arb & $\mathrm{ScHe}$ & MsTr & $\mathrm{X}$ & SMn & + & - & - & - & - \\
\hline Urticaceae & Urtica diocica L. & G & Per & $\mathrm{He}-\mathrm{Sc}$ & $\operatorname{MsMgTr}$ & $\begin{array}{l}\text { XMs- } \\
\text { MsHg }\end{array}$ & SilRu & + & + & + & + & - \\
\hline Urticaceae & U. galeopsifolia Wierzb. ex Opiz & $\mathrm{HKr}$ & Per & $\mathrm{HeSc}$ & $\operatorname{MgTr}$ & Ms-Hg & PalSil & + & + & - & - & - \\
\hline Valerianaceae & Valeriana officinalis L. & $\mathrm{HKr}$ & Per & $\mathrm{ScHe}$ & $\operatorname{MgTr}$ & $\mathrm{HgMs}$ & $\mathrm{SMnPr}$ & + & - & - & - & RLD3 \\
\hline Valerianaceae & V. stolonifera Czem. & $\mathrm{HKr}$ & Per & $\mathrm{ScHe}$ & MsMgTr & $\begin{array}{l}\text { XMs- } \\
\text { HgMs }\end{array}$ & PrSMnSil & + & - & - & - & RLD3 \\
\hline Valerianaceae & Valerianella carinata Loisel. & $\mathrm{T}$ & Ann & $\mathrm{He}$ & $\mathrm{MsOgTr}$ & Ms & RuPtPs & + & + & - & - & - \\
\hline Violaceae & Viola ambigua Walldst. et Kit. & $\mathrm{HKr}$ & Per & $\mathrm{ScHe}$ & MsTr & MsX & SMnPtSt & + & + & + & - & - \\
\hline Violaceae & V. arvensis Murray & $\mathrm{HKr}$ & Per & $\mathrm{ScHe}$ & OgMsTr & Ms & SilPsRu & + & - & - & - & Adv \\
\hline Violaceae & $V$. canina $\mathrm{L}$. & $\mathrm{HKr}$ & Per & $\mathrm{ScHe}$ & MsTr & Ms & PrSil & + & + & - & - & - \\
\hline Violaceae & V. hirta L. & $\mathrm{HKr}$ & Per & $\mathrm{ScHe}$ & $\operatorname{MsMgTr}$ & MsX-Ms & StSil & + & + & - & - & - \\
\hline Violaceae & V. lavrencoana Klokov & $\mathrm{T}$ & Ann & $\mathrm{ScHe}$ & OgTr & XMs & RuSilPs & + & + & - & - & - \\
\hline Violaceae & V. matutina Klokov & $\mathrm{HKr}$ & Ann & $\mathrm{ScHe}$ & MsTr & $\mathrm{XMs}$ & RuSMnPr & + & + & - & - & - \\
\hline Violaceae & V. mirabilis L. & $\mathrm{HKr}$ & Per & $\mathrm{HeSc}$ & MsMgTr & Ms & Sil & + & + & - & - & - \\
\hline Violaceae & V. odorata L. & $\mathrm{HKr}$ & Per & $\mathrm{HeSc}$ & MsMgTr & Ms & RuSil & + & + & - & - & - \\
\hline Violaceae & V.rupestris F. W. Schmidt & $\mathrm{HKr}$ & Ann & $\mathrm{ScHe}$ & OgMsTr & XMs & PtSilPs & + & + & - & - & - \\
\hline Violaceae & V. suavis M. Bieb. & $\mathrm{HKr}$ & Per & $\mathrm{HeSc}$ & MsTr & $\mathrm{HgMs}$ & PrSil & + & + & - & - & - \\
\hline Violaceae & V. tricolor L. & $\mathrm{HKr}$ & Ann & $\mathrm{ScHe}$ & MsTr & MsX & RuSMnPr & + & + & - & - & - \\
\hline Vitaceae & Partenocissus quinquefolia (L.) Planch. & $\mathrm{nPh}$ & $\mathrm{Fr}$ & $\mathrm{ScHe}$ & MsTr & Ms & SilCulRu & - & - & + & + & Adv, Inv \\
\hline Vitaceae & Vitis labrusca L. & $\mathrm{nPh}$ & $\mathrm{Fr}$ & $\mathrm{ScHe}$ & $\mathrm{MsTr}$ & Ms & SilCulRu & - & - & - & + & Adv \\
\hline Vitaceae & V. vinifera C.C. Gmel. & $\mathrm{nPh}$ & $\mathrm{Fr}$ & $\mathrm{ScHe}$ & $\mathrm{MsTr}$ & Ms & SilCuIRu & - & - & - & + & Adv \\
\hline
\end{tabular}

Notes: Ann (Annuus) - annuals, Bien (Biennis) - biennials, Per (Perennis) - perennials, SFr (Suffrutex) - shrub, Fr (Frutex) - shrub, Arb (Arbor) - tree; climamorphs: Ph phanerophyte, $\mathrm{HKr}$ - hemicryptophyte, $\mathrm{Kr}$ - cryptophyte, $\mathrm{G}$ - geophyte, $\mathrm{T}$ - therophyte; heliomorphs: He (Heliophiton) - heliophyte (light lovers), Sc (Sciophiton) - sciophyte (shade-bearing); tropomorphs: OgTr (Oligotroph) - oligotroph (plant of nutrient-poor soils), MsTr (Mesotroph) - mesotroph (medium-rich soils), MgTr (Megatroph) - megatroph (plant of nutrient-rich soils); hygromorphs: Hy (Hydatophyton) - hydatophyte (submerged), Pl (Pleistophyton) - pleistophyte (with floating leaves), Hel (Helophyton) helophyte (air-water), $\mathrm{Hg}$ (Hygrophyton) - hygrophyte (humid habitats), Ms (Mesophyton) - mesophyte (habitats of average moisture), X (Xerophyton) - xerophyte (dry habitats); cenomorphs: Aq (Aqant) - aquant (aquatic), Pal (Paludosus) - paludant (marsh), Pr (Pratensis) - pratant (meadow), Sil (Silvaticus) - sylvant (forest), St (Stepposus) stepant (steppe), SMn (Margosilvaticus) - sylvomargoant (forest species), Ps (Psammophyton) - psamophant (type of sandy soils), Pt (Petrophyton) - petrant (type of stony soils), $\mathrm{Ru}$ (Ruderatus) - ruderant (weedy), Hal (Halophyton) - halophant (a type of saline soils and reservoirs with high mineralization), $\mathrm{Cu}$ (Cultus) - a cultivator (a species that is cultivated); others: Par (Parasitus) - parasite, r (Radicatus) - rooted, er (Eradicatus) - unrooted, Adv - adventive species, Inv - invasive species. RLD - Red List of Dnipropetrovsk Region ( 0 - extinct (a species about which there is no data on existence in the wild for about 50 years), 1 - endangered, 2 - vulnerable, 3 - rare, 4 - undefined (not enough information about the species or status it needs to be clarified), RBU - Red Data Book of Ukraine (E - endangered, B - vulnerable, R - rare, U - undefined. ERL - European Red List ( - rare, I- undefined).

Aquatic vegetation. The shallow waters are taken over by large thickets of Phragmites australis (Cav.) Trin. ex Steud. They occupy up to $90 \%$ of the water area and are often bordered by ribbon-like phytocenoses of Typha angustifolia $\mathrm{L}$. and other aerial-aquatic plants and submerged vegetation. Areas with the the depth of more than $2 \mathrm{~m}$ and the water areas between them are occupied by groups of Ceratophyllum demersum L., often with layers of free-floating plants: Lemna minor L., Spirodela polirrhyza (L.) Schleid., Hydrocharis morsus-ranae L. Numerous branches of the Samara and its tributaries (Samarchuk and Peschanka) have submerged vegetation with a predominance of more rheophilic species: Po- 
tamogeton perfoliatus L., P. crispus L., P. pectinatus L. There is a clear advantage of the thickets of Phragmites australis (Cav.) Trin. ex Steud., which make up about $80 \%$ of the overgrown water area. The Novoselovsky site is located just below, in the area near Novoselovka, and covers the water area with a more significant level of flooding, where there are rare wooded islands, and where there are thickets of Phragmites australis (Cav.) Trin. ex Steud.and Typha angustifolia L. which alternate with water areas partially occupied by a cenosis of submerged plants (mainly by phytocenoses of Ceratophyllum sp. and $P$. pectinatus L.).

Meadow and meadow-bog vegetation is represented by complexes of associations of mesophytes and hygrophytes. Mesophytic vegetation is represented mainly by associations with the predominance of Poa pratensis L. and Elytrigia repens (L.) Nevski with the participation of such species as Trifolium repens L., Taraxacum officinale L., Urtica diocica L., Anthriscus sylvestris (L.) Hoffm., Arctium lappa L., Achillea submillefolium Klok. et Krytzca and others. In the economic zone, in the herbage of meadows, a more significant percentage is occupied by ruderal and ruderal-adventive species: Bidens tripartita L., Ambrosia artemisifolia L., Xanthium strumarium L., X. albinum (Widd.) H. Scholtz, Artemisia absinthium L., Iva xanthifolia Nutt. There are also semi-natural groups of spontaneously natural origin comprising Elaeagnus angustifolia L. On the plots used for hayfields, xerophytization of meadows was observed, accompanied by a change in mesophilic forbs by weeds and resistant species: Tanacetum vulgare L., Cirsium ucrainicum L., Carduus crispus L., C. acanthoides L., Artemisia vulgaris L., Lactuca serriola Tomer, Berteroa incana (L.) DC., Chenopodium album L., etc. In areas of the floodplain with increased soil mineralization, among meadow vegetation, there were groups with predominating Festuca regeliana Pavl. and Carex distans L. These cenoses include: Juncus gerardi Loisel., Althea officinalis L., Scorzonera parviflora Jacq., Triglochin maritimum L. and others. On wet and swampy meadows, the groups of creeping bent Agrostis stolonifera L., Poa palustris L., Bolboschoenus maritimus (L.) Palla dominate with hygrophilic species such as Lycopus europaeus L., Ranunculus repens L., R. sceleratus L., Rumex crispus L., Carex vulpina L., Eleocharis palustris (L.) Roem. et Schult, Potentilla anserina L., Heracleum sibiricum L., Symphytum officinale L., Atriplex nitens Schkuhr, Mentha aquatica $\mathrm{L}$. In the Samara valley, where water bodies were gradually swamped and overgrown with air-aquatic vegetation, swamps have developed in the floodplain. Most of them are dominated by Phragmites australis (Cav.) Trin. ex Steud. including such wetland species as Alisma plantago-aquatica L. With., Oenanthe aquatica (L.) Poir.), Sium latifolium L., Butomus umbellatus L., etc.

Trees and shrubs of the Samara Plavni Regional Landscape Park are represented by natural forest complexes, semi-natural groupings of spontaneous natural origin and man-made plantations. Natural oak forest com- plexes are localized in floodplain habitats and belong to short-lived forest types. They are represented by a fairly wide range of short-lived forests in flooded habitats: pure oak forests, birch bark oak forests, birch-ash oak forests, elm-ash oak forests, linden oak forests. Natural tree and shrub vegetation is localized exclusively in the protected area in its northern part along the river. From the lower limit of the city of Novomoskovsk to the village of Peschanka, the Samara is represented mainly by the Querceta roburi groupings. Natural oak forests are of a derived type and have a mosaic pattern of spatial distribution. They are distinguished by one-, twotiered low-growing stands. The floristic structure of the stand of natural oak forests of the Samara floodplain in the territory of the Samara Plavni Regional Landscape Park consists of typical short-lived tree species: $Q u$ ercus robur L. (completely dominates in the species composition of natural oak forests), Ulmus minor Mill., Ulmus laevis Pall., Fraxinus excelsior L., Acer tataricum L. (in arboreal and bush form), less often Tilia cordata Mill., Pyrus communis L., Acer campestre L., Acer platanoides L., Ulmus suberosa Moench (singly). Populus alba L., Populus nigra L., and Salix $a l b a \mathrm{~L}$. are added to fresh and wet locations of the oak forest complex as local fragments of associations. Sometimes, under the canopy of natural oak forests, especially in the flooded areas of the Samara floodplains, groups of the adventive species Elaeagnus angustifolia L. wedge in. The shrub undergrowth in the natural oak forests is represented by the following native species: Euonymus europaea L., E. verrucosa Scop., Rhamnus cathartica L., Swida sanquinea (L.), Crataegus leiomonogyna Klok., Crataegus fallacina Klok., Sambucus nigra L., Rubus caesius L. Cenopopulations of Vincetoxicum scandens Somm. et Levier., which is included in the Red List of Dnepropetrovsk region. Acer negundo L., Morus $a l b a \mathrm{~L}$. and Elaeagnus angustifolia $\mathrm{L}$. belong to the adventive fraction of natural oak forests. The composition of artificial plantations in the territory of the Samara Plavni Regional Landscape Park, including the recreational zones, contains the following species: Robinia pseudacacia L., Ulmus minor Mill., Quercus robur L., Ulmus pumila L., Acer negundo L., Populus nigra L., Fraxinus excelsior L., Pinus sylvestris L. (young specimens planted by local residents), Rhus typhina L., Hippophae rhamnoides L. A significant number of them are involved in the processes of spontaneous growth in the territory due to seed undergrowth and vegetative growth. The dendroflora of the regulated recreation and economic zones is also enriched with fruit and ornamental species.

Aquatic invertebrates. The fauna of zoobenthos in the Samara Plavni Regional Landscape Park includes 151 species (Table 3). They include 4 species from the Red Book of Ukraine (2009) and the Red List of Dnepropetrovsk Oblast (2011). Among the various groups of benthos, phytophilic (96 species) and pelophilic (51 species) dominate. Lithophilic, psamophilic and polytopic are represented by several species (Table 3).

Table 3

Distribution of aquatic invertebrates of the Samara Plavni Regional Landscape Park by zones

\begin{tabular}{|c|c|c|c|c|c|c|c|}
\hline \multirow[b]{2}{*}{ Families } & \multirow[b]{2}{*}{ Species } & \multirow[b]{2}{*}{$\begin{array}{c}\text { Environmental } \\
\text { groups }\end{array}$} & \multicolumn{5}{|c|}{ Zones of the Regional Landscape Park } \\
\hline & & & reserved & recreational & $\begin{array}{l}\text { stationary } \\
\text { recreation }\end{array}$ & economic & $\begin{array}{c}\text { rare, adventive, } \\
\text { species }\end{array}$ \\
\hline Aeshnidae & Aeshna grandis Linnaeus, 1758 & $\mathrm{P}$ & + & + & + & + & - \\
\hline Aeshnidae & A. viridis Eversmann, 1836 & $\mathrm{Ph}$ & + & + & + & - & - \\
\hline Aeshnidae & Anax imperator Leach, 1815 & $\mathrm{Ph}$ & + & - & - & - & RBU \\
\hline Ampharetidae & Hypania invalida (Grube, 1860$)$ & $\mathrm{P}$ & + & + & + & - & - \\
\hline Asellidae & Asellus aquaticus (Linnaeus, 1758) & $\mathrm{Ph}$ & + & + & + & + & - \\
\hline Astacidae & Astacus leptodactylus Eschscholtz, 1823 & $\mathrm{Ph}$ & + & + & - & - & - \\
\hline Baetidae & Baetis rhodani (Pictet,1843) & $\mathrm{Ph}$ & + & + & + & - & - \\
\hline Baetidae & Cloeon dipterum (Linnaeus, 1761) & $\mathrm{Ph}$ & + & + & + & + & - \\
\hline Baetidae & C. simile Eaton, 1870 & $\mathrm{Ph}$ & + & + & - & - & - \\
\hline Bithyniidae & Bithynia leachi (Sheppard, 1823) & $\mathrm{Ph}$ & + & + & + & - & - \\
\hline Bithyniidae & B. tentaculata (Linnaeus, 1758) & $\mathrm{Ph}$ & + & + & + & + & - \\
\hline Caenidae & Caenis horaria (Linnaeus, 1758) & $\mathrm{Ph}$ & + & + & + & + & - \\
\hline Caenidae & C. macrura Stephens, 1836 & $\mathrm{Ph}$ & + & + & + & - & - \\
\hline Calopterygidae & Agrion virgo (Linnaeus, 1758) & $\mathrm{Ph}$ & + & + & - & - & - \\
\hline Cardiidae & Monodacna colorata (Eichwald, 1829) & $\mathrm{P}$ & + & + & - & - & - \\
\hline Ceratopogonidae & Bezzia sp. & $\mathrm{P}$ & + & - & + & + & - \\
\hline Ceratopogonidae & Ceratopogon sp. & $\mathrm{P}$ & + & - & - & - & - \\
\hline Ceratopogonidae & Culicoides (Monoculicoides) riethi Kieffer, 1914 & $\mathrm{Ph}$ & + & + & + & - & - \\
\hline Chaoboridae & Chaoborus sp. & $\mathrm{P}$ & + & + & + & + & - \\
\hline Chironomidae & Ablabesmyia monilis (Linnaeus, 1758) & $\mathrm{Ph}$ & + & + & - & - & - \\
\hline
\end{tabular}




\begin{tabular}{|c|c|c|c|c|c|c|c|}
\hline \multirow[b]{2}{*}{ Families } & \multirow[b]{2}{*}{ Species } & \multirow{2}{*}{$\begin{array}{l}\text { Environmental } \\
\text { groups }\end{array}$} & \multicolumn{5}{|c|}{ Zones of the Regional Landscape Park } \\
\hline & & & reserved & recreational & $\begin{array}{l}\text { stationary } \\
\text { recreation }\end{array}$ & economic & $\begin{array}{c}\text { rare, adventive, } \\
\text { species }\end{array}$ \\
\hline Chironomidae & Chironomus plumosus (Linnaeus, 1758) & $\mathrm{P}$ & + & + & + & + & - \\
\hline Chironomidae & Corynoneura celeripes Winnertz, 1852 & $\mathrm{P}$ & + & - & - & - & - \\
\hline Chironomidae & Cricotopus algarum (Kieffer, 1911) & $\mathrm{Ph}$ & + & - & - & - & - \\
\hline Chironomidae & C. sylvestris (Fabricius, 1794) & $\mathrm{Ph}$ & + & - & - & - & - \\
\hline Chironomidae & Cryptochironomus defectus (Kieffer, 1913) & $\mathrm{P}$ & + & + & + & - & - \\
\hline Chironomidae & Endochironomus albipennis (Meigen, 1830) & $\mathrm{P}$ & + & + & - & - & - \\
\hline Chironomidae & Synendotendipes dispar (Meigen, 1830) & $\mathrm{P}$ & + & + & + & + & - \\
\hline Chironomidae & Endochironomus tendens (Fabricius, 1775) & $\mathrm{Ph}$ & + & + & + & - & - \\
\hline Chironomidae & Glyptotendipes cauliginellus (Kieffer, 1913) & $\mathrm{Ph}$ & + & + & + & + & - \\
\hline Chironomidae & Dicrotendipes nervosus (Staeger, 1839) & $\mathrm{P}$ & + & + & + & + & - \\
\hline Chironomidae & Microtendipes chloris (Meigen, 1818) & $\mathrm{P}$ & + & + & - & - & - \\
\hline Chironomidae & Tanypus punctipennis Meigen, 1818 & $\mathrm{P}$ & + & + & + & - & - \\
\hline Chironomidae & Polypedilum nubeculosum (Meigen, 1804) & $\mathrm{P}$ & + & + & + & + & - \\
\hline Chironomidae & P. scalaenum (Schrank, 1803) & $\mathrm{P}$ & + & + & - & - & - \\
\hline Chironomidae & Psectrocladius psilopterus (Kieffer, 1906) & $\mathrm{Ph}$ & + & + & + & + & - \\
\hline Chironomidae & Procladius choreus (Meigen, 1804) & $\mathrm{P}$ & + & + & + & + & - \\
\hline Chironomidae & Tanytarsus gregarius Kieffer, 1909 & $\mathrm{P}$ & + & - & - & + & - \\
\hline Chironomidae & Cladotanytarsus mancus (Walker, 1856) & $\mathrm{P}$ & + & + & + & - & - \\
\hline Coenagrionidae & Coenagrion pulchellum Vander Linden, 1825 & $\mathrm{Ph}$ & + & + & - & - & - \\
\hline Coenagrionidae & Ischnura elegans Vander Linden, 1820 & $\mathrm{Ph}$ & + & + & - & - & - \\
\hline Corixidae & Sigara striata (Linnaeus, 1758) & $\mathrm{Ph}$ & + & + & - & - & - \\
\hline Corophiidae & Corophium curvispinum G. O. Sars, 1895 & $\mathrm{P}$ & + & + & + & - & - \\
\hline Crambidae & Parapoynx stratiotata (Linnaeus, 1758) & $\mathrm{Ph}$ & + & + & - & - & - \\
\hline Cristatellidae & Cristatella mucedo Cuvier, 1798 & $\mathrm{Ph}$ & + & - & - & - & - \\
\hline Culicidae & Anopheles sp. & $\mathrm{Ph}$ & + & + & + & + & - \\
\hline Dreissenidae & Dreissena bugensis Andrusov, 1897 & $\mathrm{~L}$ & + & + & + & + & - \\
\hline Dreissenidae & D. polymorpha (Pallas, 1771) & $\mathrm{P}$ & + & + & + & - & - \\
\hline Dytiscidae & Cybister lateralimarginalis (De Geer, 1774) & $\mathrm{Ph}$ & + & + & - & - & - \\
\hline Dytiscidae & Dytiscus marginalis Linnaeus, 1758 & $\mathrm{Ph}$ & + & + & + & - & - \\
\hline Dytiscidae & Laccophilus hyalinus (De Geer, 1774) & $\mathrm{Ph}$ & + & + & + & - & - \\
\hline Ecnomidae & Ecnomus tenellus (Rambur, 1842) & $\mathrm{Ph}$ & + & + & + & + & - \\
\hline Ephemeridae & Ephemera vulgata Linnaeus, 1758 & $\mathrm{Ph}$ & + & + & - & - & - \\
\hline Gammaridae & Echinogammarus ischnus (Stebbing, 1899) & $\mathrm{Ph}$ & + & - & - & - & - \\
\hline Gammaridae & E. warpachowskyi Sars, 1897 & $\mathrm{Ph}$ & + & + & - & - & - \\
\hline Gammaridae & Dikerogammarus haemobaphes (Eichwald, 1841) & $\mathrm{P}, \mathrm{Ps}$ & + & + & + & - & - \\
\hline Gammaridae & D. villosus (Sowinsky, 1894) & $\mathrm{P}, \mathrm{Ps}$ & + & + & + & - & - \\
\hline Gammaridae & Obesogammarus crassus (Sars, 1894) & $\mathrm{Ph}$ & + & + & - & - & - \\
\hline Gammaridae & Gammarus pulex (Linnaeus, 1758) & $\mathrm{Ph}$ & + & - & - & - & - \\
\hline Gammaridae & Euxinia maeoticus (Sovinskij, 1894) & $\mathrm{Ph}$ & + & + & - & - & - \\
\hline Gammaridae & Obesogammarus obesus (Sars, 1894) & $\mathrm{Ph}$ & + & + & - & - & - \\
\hline Gammaridae & Pontogammarus robustoides (Sars, 1894) & $\mathrm{Ph}$ & + & + & - & - & - \\
\hline Gerridae & Aquarius paludum (Fabricius, 1794) & $\mathrm{Ph}$ & + & + & - & - & - \\
\hline Glossiphoniidae & Batracobdella paludosa (Carena, 1824) & $\mathrm{Ph}$ & + & - & - & - & - \\
\hline Glossiphoniidae & Glossiphonia complanata (Linnaeus, 1758) & $\mathrm{Ph}$ & + & + & - & + & - \\
\hline Glossiphoniidae & Helobdella stagnalis (Linnaeus, 1758) & $\mathrm{Ph}$ & + & - & - & - & - \\
\hline Gomphidae & Gomphus vulgatissimus Linnaeus, 1758 & $\mathrm{Ph}$ & + & + & - & - & - \\
\hline Gyrinidae & Gyrinus marinus Gyllenhal, 1808 & $\mathrm{Ph}$ & + & + & - & - & - \\
\hline Haliplidae & Haliplus flavicollis Sturm, 1834 & $\mathrm{Ph}$ & + & + & - & - & - \\
\hline Haliplidae & H. fluviatilis Aube, 1836 & $\mathrm{Ph}$ & + & + & + & - & - \\
\hline Haliplidae & H. ruficollis (De Geer, 1774) & $\mathrm{Ph}$ & + & + & + & - & - \\
\hline Heptageniidae & Heptagenia sulphurea (Müller, 1776) & $\mathrm{Ph}$ & + & + & - & - & - \\
\hline Hirudinidae & Hirudo medicinalis Linnaeus, 1758 & $\mathrm{Ph}$ & + & - & - & - & RBU \\
\hline Hydridae & Hydra vulgaris Pallas, 1766 & $\mathrm{P}$ & + & + & - & - & - \\
\hline Hydrobiidae & Lithoglyphus naticoides (C. Pfeiffer, 1828) & $\mathrm{Ph}$ & + & + & + & + & - \\
\hline Hydrodromidae & Hydrodroma despiciens (Muller, 1776) & $\mathrm{Ph}$ & + & + & + & - & - \\
\hline Hydrophilidae & Berosus luridus (Linnaeus, 1760) & $\mathrm{Ph}$ & + & + & - & - & - \\
\hline Hydrophilidae & Enochrus quadripunctatus (Herbst, 1797) & $\mathrm{Ph}$ & + & + & + & - & - \\
\hline Hydrophilidae & Hydrobius fuscipes (Linnaeus, 1758) & $\mathrm{Ph}$ & + & - & - & - & - \\
\hline Hydrophilidae & Hydrous piceus (Linnaeus, 1758) & $\mathrm{Ph}$ & + & + & - & - & - \\
\hline Hydropsychidae & Hydropsyche ornatula McLachlan, 1878 & $\mathrm{Ph}$ & + & + & + & - & - \\
\hline Hydroptilidae & Agraylea multipunctata Curtis, 1834 & $\mathrm{Ph}$ & + & + & - & - & - \\
\hline Janiridae & Jaera sarsi Valcanov, 1936 & $\mathrm{~L}$ & + & + & - & - & RBU \\
\hline Leptoceridae & Athripsodes cinereus (Curtis, 1834) & $\mathrm{Ph}$ & + & + & + & - & - \\
\hline Leptoceridae & Triaenodes bicolor (Curtis, 1834) & $\mathrm{Ph}$ & + & + & - & - & - \\
\hline Libellulidae & Libellula depress $a$ Linnaeus, 1758 & $\mathrm{P}$ & + & + & + & + & - \\
\hline Limnesiidae & Limnesia maculata (Muller, 1776) & $\mathrm{Ph}$ & + & + & - & - & - \\
\hline Lumbriculidae & Lumbriculus variegatus (Müller, 1774) & $\mathrm{P}$ & + & + & + & + & - \\
\hline Lymnaeidae & Limnaea stagnalis (Linnaeus, 1758) & $\mathrm{Ph}$ & + & + & + & + & - \\
\hline Lymnaeidae & Omphiscola glabra (O.F. Müller, 1774) & $\mathrm{Ph}$ & + & + & - & - & - \\
\hline Lymnaeidae & Radix auricularia (Linnaeus, 1758) & $\mathrm{Ph}$ & + & + & + & + & - \\
\hline Lymnaeidae & R. balthica (Linnaeus, 1758 ) & $\mathrm{Ph}$ & + & + & + & - & - \\
\hline Lymnaeidae & Stagnicola palustris (O. F. Müller, 1774) & $\mathrm{Ph}$ & + & + & - & - & - \\
\hline Melanopsidae & Fagotia esperi (A. Ferussac, 1823) & $\mathrm{Ph}$ & + & + & - & - & - \\
\hline Mesoveliidae & Mesovelia furcata Mulsant \& Rey, 1852 & $\mathrm{Ph}$ & + & + & - & - & - \\
\hline Molannidae & Molanna angustata Curtis, 1834 & $\mathrm{Ph}$ & + & + & - & - & - \\
\hline
\end{tabular}




\begin{tabular}{|c|c|c|c|c|c|c|c|}
\hline \multirow[b]{2}{*}{ Families } & \multirow[b]{2}{*}{ Species } & \multirow[b]{2}{*}{$\begin{array}{c}\text { Environmental } \\
\text { groups }\end{array}$} & \multicolumn{5}{|c|}{ Zones of the Regional Landscape Park } \\
\hline & & & reserved & recreational & $\begin{array}{l}\text { stationary } \\
\text { recreation }\end{array}$ & economic & $\begin{array}{l}\text { rare, adventive, } \\
\text { species }\end{array}$ \\
\hline Mysidae & Katamysis warpachowskyi Sars, 1893 & $\mathrm{P}, \mathrm{Ph}$ & + & - & - & - & RBU, Adv \\
\hline Mysidae & Limnomysis benedeni Czerniavsky, 1882 & $\mathrm{Ph}$ & + & + & + & - & - \\
\hline Mysidae & Paramysis lacustris (Czerniavsky, 1882) & $\mathrm{Ph}$ & + & + & - & - & - \\
\hline Naididae & Ophidonais serpentina (O. F. Müller, 1773) & $\mathrm{P}$ & + & + & - & - & - \\
\hline Naididae & Stylaria lacustris (Linnaeus, 1767) & $\mathrm{Ph}$ & + & + & + & + & - \\
\hline Naididae & Uncinais uncinata (Orsted, 1842) & $\mathrm{P}$ & + & + & + & - & - \\
\hline Naucoridae & Ilyocoris cimicoides (Linnaeus, 1758) & $\mathrm{Ph}$ & + & + & + & + & - \\
\hline Nepidae & Ranatra linearis (Linnaeus, 1758) & $\mathrm{Ph}$ & + & + & + & + & - \\
\hline Nepoidea & Nepa cinerea Linnaeus, 1758 & $\mathrm{Ph}$ & + & + & + & - & - \\
\hline Neritidae & Theodoxus fluviatilis (Linnaeus, 1758) & $\mathrm{L}$ & + & + & + & - & - \\
\hline Notonecta & Notonecta glauca Linnaeus, 1758 & $\mathrm{Ph}$ & + & + & + & + & - \\
\hline Oligoneuriidae & Oligoneuriella rhenana (Imhoff, 1852) & $\mathrm{Ph}$ & + & + & - & - & - \\
\hline Phryganeidae & Phryganea grandis Linnaeus, 1758 & $\mathrm{Ph}$ & + & + & + & - & - \\
\hline Physidae & Physella acuta (Drapamaud, 1805) & $\mathrm{Ph}$ & + & + & - & - & - \\
\hline Pionidae & Piona coccinea (Koch, 1836) & $\mathrm{Ph}$ & + & + & + & - & - \\
\hline Piscicolidae & Piscicola geometra (Linnaeus, 1761) & $\mathrm{Ph}$ & + & + & - & - & - \\
\hline Planorbidae & Ancylus fluviatilis O. F. Müller, 1774 & $\mathrm{Ph}$ & + & + & - & - & - \\
\hline Planorbidae & Planorbarius corneus (Linnaeus, 1758) & $\mathrm{P}, \mathrm{Ph}$ & + & + & + & + & - \\
\hline Planorbidae & Planorbis planorbis (Linnaeus, 1758) & Poly & + & + & + & + & - \\
\hline Platycnemididae & Platycnemis pennipes Pallas, 1771 & $\mathrm{Ph}$ & + & + & - & - & - \\
\hline Pleidae & Plea atomaria (Pallas, 1771) & $\mathrm{Ph}$ & + & + & + & - & - \\
\hline Plumatellidae & Plumatella fungosa (Pallas, 1768) & $\mathrm{Ph}$ & + & + & - & - & - \\
\hline Polycentropodidae & Cyrnus flavidus McLachlan, 1864 & $\mathrm{Ph}$ & + & + & + & + & - \\
\hline Polycentropodidae & Neureclipsis bimaculata (Linnaeus, 1758) & $\mathrm{Ph}$ & + & + & + & + & - \\
\hline Polycentropodidae & Polycentropus flavomaculatus (Pictet, 1834) & $\mathrm{Ph}$ & + & + & + & - & - \\
\hline Pseudocumatidae & Pseudocuma cercarioides Sars, 1894 & $\mathrm{Ph}$ & + & + & + & - & - \\
\hline Sialidae & Sialis lutaria (Linnaeus, 1758) & $\mathrm{P}$ & + & + & - & - & - \\
\hline Sisyridae & Sisyra fuscata (Fabricius, 1793) & $\mathrm{Ph}$ & + & + & - & - & - \\
\hline Sphaeriidae & Pisidium amnicum (O. F. Müller, 1774) & Ps & + & + & - & - & - \\
\hline Sphaeriidae & P. casertanum (Poli, 1791) & Ps & + & + & - & - & - \\
\hline Sphaeriidae & P. supinum Schmidt, 1850 & $\mathrm{P}$ & + & + & - & - & - \\
\hline Sphaeriidae & Sphaerium corneum (Linnaeus, 1758) & $\mathrm{Ph}$ & + & + & + & - & - \\
\hline Spongillidae & Spongilla lacustris (Linnaeus, 1758) & $\mathrm{P}$ & + & + & - & - & - \\
\hline Stratiomyidae & Stratiomys sp. & $\mathrm{Ph}$ & + & + & + & + & - \\
\hline Syrphidae & Eristalis sp. & $\mathrm{Ph}$ & + & + & + & + & - \\
\hline Tabanidae & Tabanus sp. & $\mathrm{Ph}$ & + & + & + & + & - \\
\hline Tubificidae & Limnodrilus claparedeanus Ratzel, 1869 & $\mathrm{P}$ & + & + & - & - & - \\
\hline Tubificidae & L. hoffmeisteri Claparède, 1862 & $\mathrm{P}$ & + & + & - & - & - \\
\hline Tubificidae & Tubifex tubifex (Müller, 1774) & $\mathrm{P}$ & + & + & + & + & - \\
\hline Unionidae & Anodonta anatina (Linnaeus, 1758) & $\mathrm{P}, \mathrm{Ps}$ & + & + & - & - & - \\
\hline Unionidae & A. cygnea (Linnaeus, 1758) & P, Ps & + & + & + & - & - \\
\hline Unionidae & Unio pictorum (Linnaeus, 1758) & Poly & + & + & + & - & - \\
\hline Unionidae & U. tumidus Philipsson, 1788 & $\mathrm{P}, \mathrm{Ps}$ & + & + & + & - & - \\
\hline Valvatidae & Valvata piscinalis (O. F. Müller, 1774) & $\mathrm{P}$ & + & + & + & - & - \\
\hline Valvatidae & V. naticina Menke, 1845 & $\mathrm{Ph}$ & + & + & - & - & - \\
\hline Viviparidae & Viviparus viviparus (Linnaeus, 1758) & $\mathrm{Ph}$ & + & + & + & + & - \\
\hline
\end{tabular}

Notes: $\mathrm{Ph}$ - phytophilic, P-pelophilic, Ps-psamophilic, L-lithophilic, Poly-polytropic, RBU-Red book of Ukraine.

The modern batracofauna (Amphibia) of the area of the Samara Plavni, which was studied, includes one species of amphibian of the Caudata group and five amphibian species of the Anura group. These species represent three different ecological groups (complexes): wetland (river, lake and wetland ecosystems), meadow and forest (artificial forest belts, Table 4). The herpetofauna (Reptilia) of the studied site was characterized by the presence of both hygrophilic reptile species and meso and xerophilic species. In total, 7 species of reptiles belonging to five ecological complexes were noted: wetland (river, lake and wetland ecosystems), meadow, forest (forest belts, artificial forest plantations), edge and steppe (Table 4). This accounts for $64.0 \%$ of 11 species of Dnepropetrovsk region. Of these species, three (almost 43\%) are included in the Red Book of Ukraine and are protected at the state level. Four species (57\%) are protected at the regional level.

Avifauna. The Regional Landscape Park Samara Plavni is one of the richest natural complexes in omithological terms. Its avifauna is composed of 210 species (Table 5). Of the 210 species recorded in the Regional Landscape Park Samara Plavni, 31 species (14.7\%) are listed in the Red Book of Ukraine. More than half of the latter are birds of the wetland complex (18 species $-8.5 \%$ ). Another $24.3 \%$ of the species composition is included in the Red Book of Dnipropetrovsk region. In general, the avifauna of the Samara floodplains is represented by six ecological complexes (Table 6): wetland, forest, edge, steppe, synanthropic, meadow.
The dominant group of avifauna species in the study area, comprising $42.4 \%$ of the total species composition, belongs to the wetland complex.

Birds associated with forest habitats make up the second important group $-24.7 \%$. The third group in terms of representation and importance is made up of forest species of birds $-18.6 \%$ of the total species composition. Other groups of birds do not reach the subdominant level and their share in the species composition is less than $10.0 \%$ : steppe species $-7.6 \%$, synanthropic species associated with human dwellings $-3.8 \%$, meadow habitat species $-2.9 \%$ of the total species composition. According to the status of residence, the most numerous group of species is nesting birds (45.2\%). If we also take into account the sedentary species (16.3\%) and those that feed in the study area, but do not nest in it (5.2\%), then the summer population of the study area makes up $66.7 \%$ of the total species composition.

The theriofauna of the areas of the Samara floodplains, which were studied, includes 31 species of mammals belonging to 6 orders and 12 families and represent four ecological groups (complexes): wetland (river, lake and wetland ecosystems), meadow, steppe and forest (Table 7). The Rodentia fauna (11 species) is characterized by the largest number of species. The second place in terms of the number of species number belongs to Insectivora -6 species from 3 families. Carnivora has 6 species from 2 families. Chiroptera is represented by five species. The smallest number of species was seen for Lagomorpha- 1 species. 
Table 4

Distribution of amphibian and reptile species (stable components of ecosystems) of the Samara Plavni Regional Landscape Park by zones

\begin{tabular}{|c|c|c|c|c|c|c|c|}
\hline \multirow{2}{*}{ Families } & \multirow{2}{*}{ Species } & \multirow{2}{*}{$\begin{array}{c}\text { Ecological groups } \\
\text { or ecological complex }\end{array}$} & \multicolumn{5}{|c|}{ Zones of the Regional Landscape Park } \\
\hline & & & reserved & recreational & stationary recreation & economic & rare, adventive, species \\
\hline Salamandridae & Lissotriton vulgaris (Linnaeus, 1758) & SilAqPal & + & + & + & - & BC3;RLD \\
\hline Bombinatoridae & Bombina bombina (Linnaeus, 1761) & SilAqPal & + & + & + & - & BC 2; RLD \\
\hline Hylidae & Hyla orientalis Bedriaga, 1890 & AqPal & + & + & + & - & $\mathrm{BC} 2 ; \mathrm{RLD}$ \\
\hline Pelobatidae & Pelobates vespertinus (Pallas, 1771) & SilStPr & - & + & + & - & $\mathrm{BC} 2 ; \mathrm{RLD}$ \\
\hline Bufonidae & Bufotes viridis (Laurenti, 1768) & SilStPr & + & + & + & + & $\mathrm{BC} 2 ; \mathrm{RLD}$ \\
\hline Ranidae & Pelophylax ridibundus Pallas, 1771 & SilAqPal & + & + & + & + & $\mathrm{BC} 3$ \\
\hline Emydidae & Emys orbicularis (Linnaeus, 1758) & AqPalSil & + & + & + & - & $\mathrm{BC} 2 ; \mathrm{RLD}$ \\
\hline Lacertidae & Lacerta agilis (Linnaeus, 1758) & SilStPrMar & + & + & + & + & $\mathrm{BC} 2$ \\
\hline Colubridae & Natrix natrix (Linnaeus, 1758) & AqPal & + & + & + & + & $\mathrm{BC} 3$ \\
\hline Colubridae & Natrix tessellata (Laurenti, 1768) & AqPal & + & + & - & - & $\mathrm{BC} 3$ \\
\hline Colubridae & Elaphe sauromates Pallas, 1811 & StMar & - & + & - & - & BC 2 RBU RLD \\
\hline Colubridae & Coronella austriaca Laurenti, 1768 & SilMar & + & + & + & - & BC 2 RBU RLD \\
\hline Viperidae & Vipera renardi Christoph, 1861 & SilStPrMar & - & + & + & 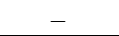 & BC 2 RBU RLD \\
\hline
\end{tabular}

Notes: Sil - species that occurs in forests, forest belts, St - in steppes, Pr - in meadows, Mar - at forest edges, Aq-Pal - in aquatic and wetland ecosystems; conservation status: KKU - the species is included in the Red Book of Ukraine, RLD - the species is included in the Red List of Dnepropetrovsk Oblast; BC ap - protected by the Beme Convention, contained in the corresponding Appendix 2 or 3.

Table 5

Species composition of the avifauna of the Regional Landscape Park Samarskie Plavni

\begin{tabular}{|c|c|c|c|c|c|c|}
\hline Families & Latin Name & Ecological Complex & Residence Status & RBU & RLD & $\mathrm{BC}$ \\
\hline Accipitridae & Accipiter gentilis (Linnaeus, 1758) & Sil & Ass & - & - & + \\
\hline Accipitridae & A. nisus (Linnaeus, 1758) & SilMn & Migr, Hib & - & - & + \\
\hline Accipitridae & Aquila clanga Pallas, 1811 & Sil & Acc & + & + & + \\
\hline Accipitridae & A. pomarina C. L. Brehm, 1831 & Sil & Acc & + & + & + \\
\hline Accipitridae & A. chrysaetos (Linnaeus, 1758) & Sil & Migr, Hib & + & + & + \\
\hline Accipitridae & Buteo lagopus (Pontoppidan, 1763) & $\mathrm{St}$ & Hib & - & - & + \\
\hline Accipitridae & B. rufinus (Cretzschmar, 1827) & Sil & T, Migr & + & + & + \\
\hline Accipitridae & B. buteo (Linnaeus, 1758) & Sil & Nest & - & - & + \\
\hline Accipitridae & Circus cyaneus (Linnaeus, 1766) & St & Migr & + & + & + \\
\hline Accipitridae & C. pygargus (Linnaeus, 1758) & St & Migr & + & + & + \\
\hline Accipitridae & C. aeruginosus (Linnaeus, 1758) & AqPal & Nest & - & - & + \\
\hline Accipitridae & Hieraaetus pennatus (Gmelin, 1788) & Sil & T, Migr & + & + & + \\
\hline Accipitridae & Haliaetus albicilla (Linnaeus, 1758) & AqPal & Ass & + & + & + \\
\hline Accipitridae & Milvus migrans (Boddaert, 1783) & Sil & T, Migr & + & + & + \\
\hline Accipitridae & Pernis apivorus (Linnaeus, 1758) & Sil & Migr & - & + & + \\
\hline Aegithalidae & Aegithalos caudatus (Linnaeus, 1758) & Sil & Ass & - & - & + \\
\hline Alaudidae & Alauda arvensis Linnaeus, 1758 & St & Nest & - & - & + \\
\hline Alaudidae & Calandrella cinerea (Gmelin, 1789) & St & Migr & - & - & + \\
\hline Alaudidae & Eremophila alpestris (Linnaeus, 1758) & St & $\mathrm{Hib}$ & - & - & + \\
\hline Alaudidae & Galerida cristata (Linnaeus, 1758) & St & Ass & - & - & + \\
\hline Alaudidae & Lullula arborea (Linnaeus, 1758) & SilMn & Nest & - & - & + \\
\hline Alaudidae & Melanocorypha calandra (Linnaeus, 1766) & St & Migr & - & - & + \\
\hline Alcedinidae & Alcedo atthis (Linnaeus, 1758) & AqPal & Nest & - & - & + \\
\hline Anatidae & Anas platyrhynchos Linnaeus, 1758 & AqPal & Nest & - & - & + \\
\hline Anatidae & A. crecca Linnaeus, 1758 & AqPal & Nest & - & - & + \\
\hline Anatidae & A. strepera Linnaeus, 1758 & AqPal & Nest & + & + & + \\
\hline Anatidae & A. penelope Linnaeus, 1758 & AqPal & Migr & - & - & + \\
\hline Anatidae & A. acuta Linnaeus, 1758 & AqPal & Migr & - & + & + \\
\hline Anatidae & A. querquedula Linnaeus, 1758 & AqPal & Nest & - & - & + \\
\hline Anatidae & A. clypeata Linnaeus, 1758 & AqPal & Migr & - & + & + \\
\hline Anatidae & Anser anser (Linnaeus, 1758) & $\mathrm{AqPal}$ & Nest & - & - & + \\
\hline Anatidae & A. albifrons (Scopoli, 1769) & AqPal & Migr & - & - & + \\
\hline Anatidae & A. fabalis (Latham, 1787) & AqPal & Migr & - & - & + \\
\hline Anatidae & Aythy a ferina (Linnaeus, 1758) & AqPal & Nest & - & - & + \\
\hline Anatidae & A. fuligula (Linnaeus, 1758) & AqPal & Migr & - & - & + \\
\hline Anatidae & A. marila (Linnaeus, 1761) & AqPal & Migr & - & - & + \\
\hline Anatidae & Bucephala clangula (Linnaeus, 1758) & AqPal & Migr & + & + & + \\
\hline Anatidae & Cygnus olor (Gmelin, 1789) & AqPal & Nest & - & - & + \\
\hline Anatidae & C. cygnus (Linnaeus, 1758) & AqPal & Migr & - & + & + \\
\hline Anatidae & C. bewickii Yarrell, 1830 & AqPal & Acc & + & + & - \\
\hline Anatidae & Netta rufina (Pallas, 1773) & $\mathrm{AqPal}$ & Nest & + & + & + \\
\hline Anatidae & Mergus albellus Linnaeus, 1758 & AqPal & Migr & - & + & + \\
\hline Anatidae & M. merganser Linnaeus, 1758 & AqPal & Migr & - & - & + \\
\hline Anatidae & Rufibrenta ruficollis (Pallas, 1769) & AqPal & Migr & + & + & + \\
\hline Anatidae & Tadorna ferruginea (Pallas, 1764) & AqPal & $\mathrm{T}$ & + & + & + \\
\hline Ardeidae & Ardea cinerea Linnaeus, 1758 & AqPal & Nest & - & - & + \\
\hline Ardeidae & A. purpurea Linnaeus, 1766 & AqPal & Nest & - & - & + \\
\hline Ardeidae & Ardeola ralloides (Scopoli, 1769) & AqPal & Nest & + & + & + \\
\hline Ardeidae & Botaurus stellaris (Linnaeus, 1758) & AqPal & Nest & - & - & + \\
\hline Ardeidae & Ciconia ciconia (Linnaeus, 1758) & AqPal & Nest & - & - & + \\
\hline Ardeidae & Egretta alba (Linnaeus, 1758) & AqPal & Nest & - & - & + \\
\hline Ardeidae & E. garzetta (Linnaeus, 1766) & AqPal & Nest & - & - & + \\
\hline Ardeidae & Lxobrychus minutus (Linnaeus, 1766) & AqPal & Nest & - & - & + \\
\hline Ardeidae & Nycticorax nycticorax (Linnaeus, 1758) & AqPal & Nest & - & - & + \\
\hline
\end{tabular}




\begin{tabular}{|c|c|c|c|c|c|c|}
\hline Families & Latin Name & Ecological Complex & Residence Status & RBU & RLD & $\mathrm{BC}$ \\
\hline Ardeidae & Platalea leucorodia Linnaeus, 1758 & AqPal & Acc & + & + & + \\
\hline Apodidae & Apus apus (Linnaeus, 1758) & Syn & T, Migr & - & - & + \\
\hline Bombycillidae & Bombycilla garrulus (Linnaeus, 1758) & Sil & $\mathrm{Hib}$ & - & - & + \\
\hline Caprimulgidae & Caprimulgus europaeus Linnaeus, 1758 & SilMn & Nest & - & - & + \\
\hline Certhiidae & Certhia familiaris Linnaeus, 1758 & Sil & Ass & - & - & + \\
\hline Charadriidae & Charadrius dubius Scopoli, 1786 & AqPal & Nest & - & - & + \\
\hline Charadriidae & Himantopus himantopus (Linnaeus, 1758) & AqPal & Migr & + & + & + \\
\hline Charadriidae & Vanellus vanellus (Linnaeus, 1758) & AqPal & Nest & - & - & + \\
\hline Columbidae & Columba palumbus Linnaeus, 1758 & SilMn & Nest & - & - & + \\
\hline Columbidae & C. oenas Linnaeus, 1758 & SilMn & Migr & + & + & + \\
\hline Columbidae & C. livia Gmelin, 1789 & Syn & Ass & - & - & + \\
\hline Columbidae & Streptopelia decaocto (Frivaldszky, 1838) & SilMn & Nest & - & - & + \\
\hline Columbidae & S. turtur (Linnaeus, 1758) & Sil & Nest & - & - & + \\
\hline Coraciidae & Coracias garrulus Linnaeus, 1758 & St & $\mathrm{M}$ & + & + & + \\
\hline Corvidae & Corvus monedula Linnaeus, 1758 & SilMn & Ass & - & - & - \\
\hline Corvidae & C. frugilegus Linnaeus, 1758 & SilMn & Ass & - & - & - \\
\hline Corvidae & C. cornix Linnaeus, 1758 & SilMn & Ass & - & - & - \\
\hline Corvidae & C. corax Linnaeus, 1758 & SilMn & Ass & - & - & + \\
\hline Corvidae & Garrulus glandarius (Linnaeus, 1758) & Sil & Ass & - & - & + \\
\hline Corvidae & Pica pica (Linnaeus, 1758) & SilMn & Ass & - & - & - \\
\hline Cuculidae & Cuculus canorus Linnaeus, 1758 & SilMn & Nest & - & - & + \\
\hline Falconidae & Falco subbuteo Linnaeus, 1758 & SilMn & Nest & - & + & + \\
\hline Falconidae & F. columbarius Linnaeus, 1758 & SilMn & $\mathrm{Hib}$ & - & - & + \\
\hline Falconidae & F. vespertinus Linnaeus, 1766 & SilMn & Migr & - & + & + \\
\hline Falconidae & F. tinnunculus Linnaeus, 1758 & SilMn & Nest & - & - & + \\
\hline Emberizidae & Emberiza calandra Linnaeus, 1758 & SilMn & Nest & - & - & + \\
\hline Emberizidae & E. citrinella Linnaeus, 1758 & SilMn & Ass & - & - & + \\
\hline Emberizidae & E. schoeniclus (Linnaeus, 1758) & AqPal & Nest & - & - & + \\
\hline Emberizidae & E. hortulana Linnaeus, 1758 & SilMn & Nest & - & - & + \\
\hline Emberizidae & Plectrophaenax nivalis (Linnaeus, 1758) & SilMn & $\mathrm{Hib}$ & - & - & + \\
\hline Fringillidae & Acanthis cannabina (Linnaeus, 1758) & SilMn & Ass & - & - & + \\
\hline Fringillidae & A. flammea (Linnaeus, 1758) & Sil & $\mathrm{Hib}$ & - & - & + \\
\hline Fringillidae & Carduelis carduelis (Linnaeus, 1758) & SilMn & Ass & - & - & + \\
\hline Fringillidae & Chloris chloris (Linnaeus, 1758) & Sil & Ass & - & - & + \\
\hline Fringillidae & Coccothraustes coccothraustes (Linnaeus, 1758) & Sil & Ass & - & - & + \\
\hline Fringillidae & Fringilla coelebs Linnaeus, 1758 & Sil & Nest & - & - & + \\
\hline Fringillidae & F. montifringilla Linnaeus, 1758 & SilMn & Migr, Hib & - & - & + \\
\hline Fringillidae & Loxia curvirostra Linnaeus, 1758 & Sil & Acc & - & - & + \\
\hline Fringillidae & Pyrrhula pyrrhula (Linnaeus, 1758) & Sil & Migr, Hib & - & - & + \\
\hline Fringillidae & Spinus spinus (Linnaeus, 1758) & Sil & $\mathrm{Hib}$ & - & - & + \\
\hline Gaviidae & Gavia stellata (Pontoppidan, 1763) & AqPal & Migr & - & + & + \\
\hline Gaviidae & G. arctica (Linnaeus, 1758) & AqPal & Migr & - & + & + \\
\hline Gruidae & Grus grus (Linnaeus, 1758) & AqPal & Migr & + & + & + \\
\hline Haematopodidae & Haematopus ostralegus Linnaeus, 1758 & AqPal & Migr & + & + & + \\
\hline Hirundinidae & Riparia riparia (Linnaeus, 1758) & AqPal & Nest & - & - & + \\
\hline Hirundinidae & Hirundo rustica Linnaeus, 1758 & Syn & Nest & - & - & + \\
\hline Hirundinidae & Delichon urbica (Linnaeus, 1758) & Syn & Nest & - & - & + \\
\hline Laridae & Chlidonias niger (Linnaeus, 1758) & AqPal & Nest & - & - & + \\
\hline Laridae & C. leucopterus (Temminck, 1815) & AqPal & Nest & - & - & + \\
\hline Laridae & C. hybrida (Pallas, 1811) & AqPal & Nest & - & - & + \\
\hline Laridae & Hydroprogne caspia (Pallas, 1770) & AqPal & Acc & + & + & + \\
\hline Laniidae & Lanius collurio Linnaeus, 1758 & SilMn & Nest & - & - & + \\
\hline Laniidae & L. minor Linnaeus, 1758 & SilMn & Nest & - & - & + \\
\hline Laniidae & L. excubitor Linnaeus, 1758 & SilMn & Migr, Hib & + & + & + \\
\hline Laridae & Larus ridibundus Linnaeus, 1766 & $\mathrm{AqPal}$ & Nest & - & - & + \\
\hline Laridae & L. cachinnans Pallas, 1811 & AqPal & Nest & - & - & - \\
\hline Laridae & L. canus Linnaeus, 1758 & AqPal & Acc & - & - & + \\
\hline Laridae & Sterna hirundo Linnaeus, 1758 & AqPal & Nest & - & - & + \\
\hline Laridae & S. albifrons Pallas, 1764 & $\mathrm{AqPal}$ & T, Migr & + & + & + \\
\hline Meropidae & Merops apiaster Linnaeus, 1758 & $\mathrm{St}$ & Migr & - & - & + \\
\hline Motacillidae & Anthus campestris (Linnaeus, 1758) & SilMn & Migr & - & + & + \\
\hline Motacillidae & A. trivialis (Linnaeus, 1758) & SilMn & Nest & - & - & + \\
\hline Motacillidae & A.s pratensis (Linnaeus, 1758) & SilMn & Migr & - & - & + \\
\hline Motacillidae & A. cervinus (Pallas, 1811) & SilMn & Migr & - & - & - \\
\hline Motacillidae & Motacilla flava Linnaeus, 1758 & St & Nest & - & - & + \\
\hline Motacillidae & M. citreola Pallas, 1776 & $\operatorname{Pr}$ & Nest & - & - & + \\
\hline Motacillidae & M. cinerea Tunstall, 1771 & $\operatorname{Pr}$ & Migr, Hib & - & - & + \\
\hline Motacillidae & M. alba Linnaeus, 1758 & $\operatorname{Pr}$ & Nest & - & - & + \\
\hline Muscicapidae & Ficedula albicollis (Temminck, 1815) & Sil & Nest & - & - & + \\
\hline Muscicapidae & F. parva (Bechstein, 1794) & Sil & Nest & - & + & + \\
\hline Muscicapidae & Muscicapa striata (Pallas, 1764) & SilMn & Nest & - & - & + \\
\hline Muscicapidae & Saxicola rubetra (Linnaeus, 1758) & St & Nest & - & - & + \\
\hline Muscicapidae & S. torquata (Linnaeus, 1766) & St & Nest & - & - & + \\
\hline Muscicapidae & Oenanthe oenanthe (Linnaeus, 1758) & St & Nest & - & - & + \\
\hline Muscicapidae & Phoenicurus phoenicurus (Linnaeus, 1758) & Syn & Nest & - & - & + \\
\hline Muscicapidae & P. ochruros (S.G.Gmelin, 1774) & Syn & Nest & - & - & + \\
\hline Muscicapidae & Erithacus rubecula (Linnaeus, 1758) & Sil & Nest & - & - & + \\
\hline Muscicapidae & Luscinia luscinia (Linnaeus, 1758) & Sil & Nest & - & - & + \\
\hline Muscicapidae & L. svecica (Linnaeus, 1758) & AqPal & Nest & - & - & + \\
\hline Muscicapidae & Turdus pilaris Linnaeus, 1758 & Sil & Ass & - & - & + \\
\hline Muscicapidae & T. merula Linnaeus, 1758 & Sil & Ass & - & - & + \\
\hline
\end{tabular}




\begin{tabular}{|c|c|c|c|c|c|c|}
\hline Families & Latin Name & Ecological Complex & Residence Status & RBU & RLD & $\mathrm{BC}$ \\
\hline Muscicapidae & T.s iliacus Linnaeus, 1766 & Sil & Migr & - & - & + \\
\hline Muscicapidae & T. philomelos C.L.Brehm, 1831 & Sil & Nest & - & - & + \\
\hline Muscicapidae & T. viscivorus Linnaeus, 1758 & Sil & M & - & + & + \\
\hline Oriolidae & Oriolus oriolus Linnaeus, 1758 & Sil & Nest & - & - & + \\
\hline Pandionidae & Pandion haliaeetus (Linnaeus, 1758) & AqPal & T, Migr & + & + & + \\
\hline Paradoxornithidae & Panurus biarmicus (Linnaeus, 1758) & AqPal & Ass & - & - & + \\
\hline Paridae & Remiz pendulinus (Linnaeus, 1758) & AqPal & Nest & - & - & + \\
\hline Paridae & Parus palustris Linnaeus, 1758 & Sil & Ass & - & - & + \\
\hline Paridae & P. ater Linnaeus, 1758 & Sil & Migr, Hib & - & - & + \\
\hline Paridae & P. caeruleus Linnaeus, 1758 & Sil & Ass & - & - & + \\
\hline Paridae & P. major Linnaeus, 1758 & Sil & Ass & - & - & + \\
\hline Passeridae & Passer domesticus (Linnaeus, 1758) & Syn & Ass & - & - & - \\
\hline Passeridae & P. montanus (Linnaeus, 1758) & Syn & Ass & - & - & - \\
\hline Phalacrocoracidae & Phalacrocorax carbo (Linnaeus, 1758) & AqPal & Nest & - & - & + \\
\hline Phalacrocoracidae & Ph. pygmaeus (Pallas, 1773) & AqPal & Acc & + & + & - \\
\hline Phasianidae & Perdix perdix (Linnaeus, 1758) & St & Ass & - & - & + \\
\hline Phasianidae & Coturnix coturnix (Linnaeus, 1758) & St & Nest & - & - & + \\
\hline Phasianidae & Phasianus colchicus Linnaeus, 1758 & SilMn & Ass & - & - & + \\
\hline Picidae & Jynx torquilla Linnaeus, 1758 & Sil & Nest & - & - & + \\
\hline Picidae & Picus canus Gmelin, 1788 & Sil & Nest & - & - & + \\
\hline Picidae & Dendrocopos major (Linnaeus, 1758) & Sil & Ass & - & - & + \\
\hline Picidae & D. syriacus (Hemprich et Ehrenberg, 1833) & Sil & Ass & - & - & + \\
\hline Picidae & D. medius (Linnaeus, 1758) & Sil & Nest & - & + & + \\
\hline Picidae & D. minor (Linnaeus, 1758) & Sil & Ass & - & - & + \\
\hline Podicipedidae & Podiceps ruficollis (Pallas, 1764) & AqPal & Nest & - & + & + \\
\hline Podicipedidae & P. nigricollis C.L.Brehm, 1831 & AqPal & Nest & - & + & + \\
\hline Podicipedidae & P. cristatus (Linnaeus, 1758) & AqPal & Nest & - & - & + \\
\hline Prunellidae & Prunella modularis (Linnaeus, 1758) & Sil & Migr & - & - & + \\
\hline Rallidae & Rallus aquaticus Linnaeus, 1758 & AqPal & Nest & - & - & + \\
\hline Rallidae & Porzana porzana (Linnaeus, 1766) & AqPal & Nest & - & - & + \\
\hline Rallidae & P. parva (Scopoli, 1769) & AqPal & Nest & - & - & + \\
\hline Rallidae & Crex crex (Linnaeus, 1758) & $\operatorname{Pr}$ & Nest & - & + & + \\
\hline Rallidae & Gallinula chloropus (Linnaeus, 1758) & AqPal & Nest & - & - & + \\
\hline Rallidae & Fulica atra Linnaeus, 1758 & AqPal & Nest & - & - & + \\
\hline Regulidae & Regulus regulus (Linnaeus, 1758) & SilMn & Migr, Hib & - & - & + \\
\hline Scolopacidae & Tringa ochropus Linnaeus, 1758 & AqPal & T, Migr & - & - & + \\
\hline Scolopacidae & T. glareola Linnaeus, 1758 & AqPal & T, Migr & - & - & + \\
\hline Scolopacidae & T. nebularia (Gunnerus, 1767) & AqPal & T, Migr & - & - & + \\
\hline Scolopacidae & T. totanus (Linnaeus, 1758 ) & AqPal & Nest & - & - & + \\
\hline Scolopacidae & T. erythropus (Pallas, 1764) & AqPal & Migr & - & - & + \\
\hline Scolopacidae & T. stagnatilis (Bechstein, 1803) & AqPal & Migr & + & + & + \\
\hline Scolopacidae & Actitis hypoleucos (Linnaeus, 1758) & AqPal & Migr & - & - & + \\
\hline Scolopacidae & Phylomachus pugnax (Linnaeus, 1758) & $\operatorname{Pr}$ & Migr & - & - & + \\
\hline Scolopacidae & Calidris minuta (Leisler, 1812) & AqPal & Migr & - & - & + \\
\hline Scolopacidae & C. temminckii (Leisler, 1812) & AqPal & Migr & - & - & + \\
\hline Scolopacidae & Calidris alpina (Linnaeus, 1758) & AqPal & Migr & - & - & + \\
\hline Scolopacidae & Lymnocryptes minimus (Brònnich, 1764) & AqPal & Migr & - & - & + \\
\hline Scolopacidae & Gallinago gallinago (Linnaeus, 1758) & AqPal & Migr & - & - & + \\
\hline Scolopacidae & Scolopax rusticola Linnaeus, 1758 & $\operatorname{Pr}$ & Migr & - & - & + \\
\hline Scolopacidae & Numenius arquata (Linnaeus, 1758) & AqPal & Migr & + & + & + \\
\hline Scolopacidae & Limosa limosa (Linnaeus, 1758) & AqPal & T, Migr & - & + & + \\
\hline Sittidae & Sitta europaea Linnaeus, 1758 & Sil & Ass & - & - & + \\
\hline Stercorariidae & Stercorarius parasiticus (Linnaeus, 1758) & AqPal & Acc & - & - & + \\
\hline Strigidae & Asio otus (Linnaeus, 1758) & Sil & Ass & - & - & + \\
\hline Strigidae & A. flammeus (Pontoppidan, 1763) & Sil & Ass & + & + & + \\
\hline Strigidae & Otus scops (Linnaeus, 1758) & Sil & Nest & + & + & + \\
\hline Strigidae & Athene noctua (Scopoli, 1769) & Sil & Ass & - & + & + \\
\hline Stumidae & Sturnus vulgaris Linnaeus, 1758 & Sil & Nest & - & - & + \\
\hline Sylviidae & Locustella luscinioides (Savi, 1824) & AqPal & Nest & - & - & + \\
\hline Sylviidae & L. fluviatilis (Wolf, 1810) & AqPal & Nest & - & + & + \\
\hline Sylviidae & Acrocephalus schoenobaenus (Linnaeus, 1758) & AqPal & Nest & - & - & + \\
\hline Sylviidae & A. agricola (Jerdon, 1845) & AqPal & Nest & - & + & + \\
\hline Sylviidae & A. palustris (Bechstein, 1798) & AqPal & Acc & - & - & + \\
\hline Sylviidae & A. scirpaceus (Hermann, 1804) & AqPal & Nest & - & - & + \\
\hline Sylviidae & A. arundinaceus (Linnaeus, 1758) & AqPal & Nest & - & - & + \\
\hline Sylviidae & Hippolais icterina (Vieillot, 1817) & Sil & Nest & - & - & + \\
\hline Sylviidae & Sylvia nisoria (Bechstein, 1795) & SilMn & Nest & - & - & + \\
\hline Sylviidae & S. atricapilla (Linnaeus, 1758) & SilMn & Nest & - & - & + \\
\hline Sylviidae & S. borin (Boddaert, 1783) & SilMn & Nest & - & - & + \\
\hline Sylviidae & S. communis Latham, 1787 & SilMn & Nest & - & - & + \\
\hline Sylviidae & S. curruca (Linnaeus, 1758) & SilMn & Nest & - & - & + \\
\hline Sylviidae & Phylloscopus trochilus (Linnaeus, 1758) & Sil & Migr & - & - & + \\
\hline Sylviidae & P. collybita (Vieillot, 1817) & Sil & Nest & - & - & + \\
\hline Sylviidae & P. sibilatrix (Bechstein, 1793) & Sil & Nest & - & - & + \\
\hline Troglodytidae & Troglodytes troglodytes (Linnaeus, 1758) & SilMn & Migr & - & - & + \\
\hline
\end{tabular}

Notes: ecological complex: AqPal (aqa-paludosus) - wetland, Sil (silvaticus) - forest, Pr (pratensis) - meadow, Syn (synanthropus) - synanthropus, St - steppe, SilMn - forest edge; residence status: Nest - breeding migratory, Migr - migratory, T - feeds in summer, but does not nest in the study area, Hib (hibernans) - wintering, Ass (assiduus) sedentary, Acc (accidentales) - migratory, accidental; status in the Red Data Book of Ukraine and the region: $\mathrm{Ne}$ - disappearing, E - missing, R - rare, Vu - vulnerable; BC listed in the annexes of the Berne Convention. 
Table 6

The ratio of the species composition of ecological complexes and groups of birds by status of residence (\%)

\begin{tabular}{|c|c|c|c|c|c|c|c|c|c|}
\hline Ecological complexes & Nesting migratory species & Feeding but do not nest & Feed, migrate & Wintering & Accidental & Migratory & Migrants, wintering & Sedentary & Total \\
\hline Wetland & 22.4 & 0.5 & 2.8 & - & 3.3 & 12.5 & - & 0.9 & 42.4 \\
\hline Forest & 8.1 & - & 1.4 & 1.4 & 1.4 & 2.4 & 1.4 & 8.6 & 24.7 \\
\hline Meadow & 1.4 & - & - & - & - & 0.9 & 0.6 & - & 2.9 \\
\hline Synanthropic & 1.9 & - & 0.5 & - & - & - & - & 1.4 & 3.8 \\
\hline Steppe & 2.9 & - & - & 0.9 & - & 2.9 & - & 0.9 & 7.6 \\
\hline Marginal & 8.5 & - & - & 0.9 & - & 2.9 & 1.9 & 4.4 & 18.6 \\
\hline Together & 45.2 & 0.5 & 4.7 & 3.2 & 4.7 & 21.6 & 3.9 & 16.2 & 100.0 \\
\hline
\end{tabular}

Table 7

Species composition of the mammalian fauna of the Samara floodplain

\begin{tabular}{|c|c|c|c|}
\hline Family & Latin name of the species & Ecological complex & Conservation status \\
\hline Talpidae & Talpa europaea Linnaeus, 1758 & PrSil & - \\
\hline Erinaceidae & Erinaceus roumanicus Barrett-Hamilton, 1900 & StPrSil & $\mathrm{BC} 3$ \\
\hline Sorecidae & Sorex araneus Linnaeus, 1758 & StPrSilSin & $\mathrm{BC} 3$ \\
\hline Sorecidae & S. minutus (Linnaeus, 1758) & SilPr & RLD, BC 3 \\
\hline Sorecidae & Crocidura leucodon (Hermann, 1780) & StAqPal & RBU, RLD, BC 3 \\
\hline Sorecidae & C. suaveolens (Pallas, 1811) & StSilSin & RLD, BC 3 \\
\hline Vespertilionidae & Eptesicus serotinus Schreber, 1774 & PrSilStSin & $\mathrm{BC} 2$ \\
\hline Vespertilionidae & Myotis daubentonii (Kuhl, 1817) & SilAqPal & RBU, RLD, BC 2 \\
\hline Vespertilionidae & Nyctalus noctula (Schreber, 1774) & Sil & RBU, RLD, BC 2 \\
\hline Vespertilionidae & Plecotus auritus (Linnaeus, 1758) & SilSin & RBU, RLD, BC 2 \\
\hline Vespertilionidae & Vespertilio murinus Linnaeus, 1758 & SilSin & RBU, RLD, BC 2 \\
\hline Canidae & Vulpes vulpes Linnaeus (1758) & StPrSil & - \\
\hline Canidae & Nyctereutes procyonoides Gray (1834) & SilAqPal & - \\
\hline Mustelidae & Martes martes Linnaeus (1758) & PrSilStSin & $\mathrm{BC} 3$ \\
\hline Mustelidae & M. foina (Erxleben, 1777) G. Fischerde Waldheim, 1817 & SilSin & $\mathrm{BC} 3$ \\
\hline Mustelidae & Mustela (Putorius) putorius (Linnaeus, 1758) & Sil & RLD, BC 2 \\
\hline Mustelidae & Lutra lutra (Linnaeus, 1758) & AqPal & RBU, RLD, BC 2 \\
\hline Leporidae & Lepus europaeus Pallas (1778) & $\mathrm{StPr}$ & $\mathrm{BC} 2$ \\
\hline Gliridae & Dryomys nitedula Pallas, 1778 & Sil & $\mathrm{BC} 3$ \\
\hline Muridae & Apodemus agrarius Pallas (1778) & StSin & - \\
\hline Muridae & Mus musculus Linnaeus (1758) & StPrSilSin & - \\
\hline Muridae & Sylvaemus flavicollis (Melchior, 1834) & PrSilSin & - \\
\hline Muridae & S. sylvaticus(Linnaeus, 1758) & PrSilSin & - \\
\hline Muridae & Micromys minutus(Pallas, 1771) & PrSilSin & - \\
\hline Muridae & Rattus norvegicus Berkenhout (1769) & SilPrSin & - \\
\hline Cricetidae & Myodes glareolus (Schreber, 1780) & Sil & - \\
\hline Cricetidae & Microtus arvalis (s. lato) (Pallas, 1778) & PrSilSin & - \\
\hline Cricetidae & Arvicola amphibius (Linnaeus, 1758) & AqPal & - \\
\hline Cricetidae & Ondatra zibethicus (Linnaeus, 1766) & AqPal & - \\
\hline Suidae & Sus scrofa (Linnaeus, 1758) & SilStPr & - \\
\hline Cervidae & Capreolus capreolus (Linnaeus, 1758) & SilPr & $\mathrm{BC} 3$ \\
\hline
\end{tabular}

Notes: $\mathrm{Ph}$ - phytophilic, P-pelophilic, Ps - psamophilic, L- lithophilic, Poly-polytopic; Sil-a species, found in forests, forest belts, St - in steppes, Pr-in meadows, Mar-at forest edges, Aq-Pal - in aquatic and wetland ecosystems; conservation status: RBU - the species is included in the Red Book of Ukraine, RLD - the species is included in the Red List of Dnepropetrovsk Oblast; BC ap - protected by the Berne Convention, contained in the corresponding Appendix 2 or 3.

\section{Discussion}

Creation of nature reserves in reservoirs is used as a tool for biodiversity conservation in many countries. According to international studies, almost $20.0 \%$ of existing large dams occur within protected areas. In more than two thirds of these cases, dams were constructed before the creation of protected areas in the past 70-300 years (Thieme et al., 2020). In our case, the Landscape Regional Park was created 70 years after the creation of the reservoir. The impact of protected area management actions is rarely assessed. The main reasons for this finding are a lack of financial resources, gaps in managerial capacity, or poor understanding of how to counter threats (Adams et al., 2015). Therefore, the presented studies are important not only for the zoning of the territory, but also for further analysis of the dynamics of biodiversity. The zoning of protected areas is also important because parts of the reservoir often become recreation areas for water and other types of tourist activities.

The creation of the Zaporozhye Reservoir caused significant changes in the hydrological regime of the valley: meadows and floodplains of forests were flooded, which led, first of all, to a change in the vegetation of the territory. Researchers in other countries also focus on this (Chakraborty, 2021). Over 70 years, a new floodplain and flooded complex have developed. Some plant species were lost due to flooding, while others recovered their populations. Today, the analysis of the flora of the regional landscape park indicates its significant richness, systematic and ecomorphic diversity despite anthropogenic transformation. The anthropogenic transformation of the territory of the regional landscape park is evidenced by the large amount of ruderal flora. Its amount significantly predominates in the regulated recreation zone, and especially in the economic zone.

The difference between the vegetation cover of shallow waters from other parts of the park lies in the prevalence of scattered centers of overgrowth and the spread of phytocenoses, rare for the area of Potamogeton lucens L. According to the known gradations of the degree of overgrowth of water bodies, the territory of the floodplains is highly overgrown (the degree of overgrowth is more than $40 \%$ ). Natural oak forests (birch-ash oak forests, elm-ash oak forests, linden oak forests) are common in the Samara riverbed zone, which is associated with a lighter texture of the soil and a deeper standing of groundwater. These natural oak forests are distinguished by significant cenotic condition and species diversity and are the most valuable dendrological objects of the park. Increase in moisture in edaphotopes downstream of the Samara River is seen in the increase in mesophilic and hygromesophilic tree, shrub and herbaceous species. In addition to the leading hydrological factor, the state of forests is influenced by the recreational load, which can be estimated as average. Also it is important that green zones near the megapolis are able to absorb pollutants and keep them from entering groundwater (buffer tank for heavy metals) (Didur et al., 2019) this is important in the manifestation of human health potential.

According to international studies, invertebrates make up the bulk of the diversity of freshwater animals, both systematically and functionally, but they are underrepresented in assessments of the effectiveness of pro- 
tected areas with a strong bias towards vertebrate monitoring (Acreman et al., 2020). Also, benthos is one of the stable elements of ecosystems.

All six amphibian species found in the study area make up $60 \%$ of the species composition of the batrachofauna of Dnepropetrovsk region $\mathrm{Bu}-$ lakhov et al., 2007). All amphibian species have a permanent residence status in their biotopes. The noted 7 species of reptiles make up $58.3 \%$ of the species diversity of the herpetofauna of Dnipropetrovsk region, which is due to the relatively high area of the site itself and the presence of various locations with suitable ecological conditions for these species. The study area is characterized by the presence of species in need of protection. All species of reptiles living in this area have a conservation status: all 7 species are protected by the Bern Convention. Of these, three species (almost 43\%) are included in the Red Book of Ukraine and are protected at the state level. Four species (57\%) are included in the Red Book of Dnipropetrovsk region and are protected at the regional level. All reptile species have a permanent residence status. Dice snake $N$. tessellata has expanded its range in the region over the recent decades (Yermolenko et al., 2016) and inhabited the studied area. The conservation of this territory will lead to an increase in the number of species due to decrease in the disturbance factor and the preservation of natural biotopes. In addition, preconditions for increasing biological diversity and monitoring of it will appear, the importance of which cannot be overestimated (Sigwart et al., 2018).

The diversity in the biotopes of the Regional Landscape Park, and a large area allow 210 species of birds to live there. Because of the flooded area, the dominant group of avifauna species belongs to the wetland complex. Habitat monitoring and the importance of protected areas inhabited mainly by wintering species of aquatic birds has been studied and confirmed by scientists from different countries (Musilová et al., 2018; Gaget et al., 2020; Pavón-Jordán et al., 2020). In the same territories, the composition of migrants can change every few years (Ponomarenko, 2005; Bulakhov et al., 2009, 2013). The share of synanthropic species is rather low and corresponds to the natural areas of the region. This indicates a low level of transformation of the studied territory and its relative preservation. In areas subject to high anthropogenic load, the proportion of synanthropic species in the species composition reaches $7-9 \%$. Within the study area, there are both wide deep-water areas and shallow and aquatic ecosystems that are rich in macrophytic vegetation. Therefore, on the one hand, there are species associated with large water surfaces (some species of anseriformes, loons and others), and on the other hand, types of water bodies that overgrow (anseriformes, herons) and species characteristic of thickets of higher aquatic vegetation (some herons, rails).

The second most important is the forest ecological complex of birds. The birds of this complex also have a fairly diverse and large territorial base. Their main habitats are floodplain forests. The forests of the study area are diverse, among them there are both dense-crowned and semiopenwork species. This leads to the fact that the forest complex even includes quite specialized species. The main basis of habitation for birds of the marginal ornithocomplexes $(18.5 \%)$ is the thickets of various shrubs along the banks. They create favourable conditions for the birds of the forest. The habitat of steppe birds comprises river banks and insular areas with remaining steppe biotopes. However, the proportion of steppe birds in the species composition is relatively small (7.6\%). Meadow birds are represented by a very small share $-2.8 \%$ of the total species composition due to the small area of integral meadows and their fragmented nature. In addition, most of these species in the study area are migrants and do not stay for a long time in the studied ecosystems. The large percentage of nesting birds indicates that the territory has a sufficiently high reproductive potential for birds and is a place for them to feed in the summer.

The main bases of residence for synanthropic species are human settlements. One-story and low-rise buildings prevail in the area, as well as various options for nursery gardens for household plots. All this creates conditions for the habitation of synanthropic birds and explains their presence in the avifauna in the study area.

The sites of the Regional Landscape Park are valuable for migratory birds. Migrants of all groups make up more than $75.2 \%$ of the total species composition. More than half of all migrants are types of the wetland complex. In winter, the avifauna is represented by sedentary species, and those that are found all year round. The share of such species in the avifauna is not very significant and the basis for such a phenomenon is the state of the topical diversity in the studied territory. In general, the indicators of avifauna protection are relatively high and are not inferior to those of the natural reserves of the region. The presence of species listed in the Red Book of Ukraine and the list of the Berne Convention requires taking into account the interests of preserving natural environments.

The territory is characterized by the presence of 17 mammal species (55\% of the mammalian species composition of the oblast), which are protected by the Berne Convention. Nine species (29\%) are included in the Red Book of Dnipropetrovsk region and are protected at the regional level. Six species (19\%) are listed in the Red Book of Ukraine and are protected at the state level (Bulakhov \& Pakhomov, 2006).

\section{Conclusion}

Within the framework of the Samara Plavni Regional Landscape Park, a floodplain-flooded complex has developed, which united forest floodplain forests, branches and tributaries of the Samara River, meadows, wetlands and the water area of the flooded complex. Due to the variety of biotopes presented, the territory has significant biodiversity and a large number of rare species that are protected in the region, the country, and Europe. According to the zoning of the Samara Plavni Regional Landscape Park, the following zones have been identified: a reserved zone, a recreational zone, a regulated recreation zone and an economic zone. The protected area has a high level of biodiversity. The recreational zone occupies the main part of the regional landscape park and is slightly inferior to the protected zone in terms of the richness of plant and animal species. Zoning of the Samara Plavni Regional Landscape Park is of great importance for optimizing the ecological balance of territories and water areas and protecting flora and fauna. With the application of the reserve regime, the disturbance factor will decrease, conditions will be created for the preservation of the habitats of mammals, including those protected by the state. Such changes will increase the implementation of the reproductive potential of all species without exception in the ecosystems studied.

To maintain the aquatic ecosystems of the Samara Plavni Regional Landscape Park in the conditions favourable for aquatic biocenoses, it is necessary to develop and implement measures (not prohibited by law) to restore their hydrological state, disturbed as a result of anthropogenic impact, first of all - clearing silted, swampy channels and mechanical mowing of reed beds.

\section{References}

Acreman, M., Hughes, K. A., Arthington, A. H., Tickner, D., \& Dueñas, M.-A. (2020). Protected areas and freshwater biodiversity: A novel systematic review distils eight lessons for effective conservation. Conservation Letters, 13(e), 12684.

Adams, V. M., Setterfield, S. A., Douglas, M. M., Kennard, M. J., \& Ferdinands, K. (2015). Measuring benefits of protected area management: Trends across realms and research gaps for freshwater systems. Philosophical Transactions of the Royal Society B, 370(1681), 20140274.

Ali, W., Javid, A., Bhukhari, S. M., Hussain, A., Hussain, S. M., \& Rafique, H. (2018). Comparison of different trapping techniques used in herpetofaunal monitoring: A review. Punjab University Joumal of Zoology, 33(1), 57-68.

Baranovski, B., Khromykh, N., Karmyzova, L., Ivanko, I., \& Lykholat, Y. (2016). Analysis of the alien flora of Dnipropetrovsk Province. Biolohichnyi Visnyk MDPU imeni Bohdana Khmelnytskoho, 66(3), 419-429.

Baranovsky, B. A. (2000). Rastitelnost ruslovogo ravninnogo vodohranilishha [Vegetation of the channel of a lowland reservoir]. Izdatelstvo Dnepropetrovskogo Universiteta, Dnepropetrovsk (in Russian).

Becken, S., \& Job, H. (2014). Protected areas in an era of global-local change. Journal of Sustainable Tourism, 22(4), 507-527.

Belgard, A. L. (1950). Lesnaja rastitelnost jugo-vostoka USSR [Forest vegetation of southeast of the Ukrainian SSR]. Izdatelstvo Kievskogo Gosudarstvennogo Universiteta, Kiev (in Russian).

Brygadyrenko, V. V. (2015). Community structure of litter invertebrates of forest belt ecosystems in the Ukrainian steppe zone. International Journal of Environmental Research, 9(4), 1183-1192.

Brygadyrenko, V. V. (2016). Evaluation of ecological niches of abundant species of Poecilus and Pterostichus (Coleoptera: Carabidae) in forests of the steppe zone of Ukraine. Entomologica Fennica, 27(2), 81-100.

Bulakhov, V. L., \& Pakhomov, O. Y. (2006). Biolohichne riznomanittia Ukrainy. Dnipropetrovska oblast. Ssavtsi (Mammalia) [Biodiversity of Ukraine. Dnipro- 
petrovsk region. Mammals (Mammalia)]. Dnipropetrovsk University Press, Dnipropetrovsk (in Ukrainian).

Bulakhov, V. L., Gasso, V. Y., \& Pakhomov, A. Y. (2007). Biolohichne riznomanittia Ukrainy. Dnipropetrovska oblast. Zemnovodni ta plazuny (Amphibia et Reptilia) [Biological Diversity of Ukraine. Dnipropetrovsk region. Amphibians and Reptiles (Amphibia et Reptilia)]. Dnipropetrovsk National University Press, Dnipropetrovsk (in Ukrainian).

Bulakhov, V. L., Hubkin, A. A., Ponomarenko, O. L., \& Pakhomov, O. Y. (2013). Biolohichne riznomanittia Ukrainy. Dnipropetrovska oblast. Ptakhy: Nehorobtsepodibni (Aves: Non-Passeriformes) [Biological diversity of Ukraine. Dnipropetrovsk region. Birds: Non-passerines]. Dnipropetrovsk National University Press, Dnipropetrovsk (in Ukrainian)

Bulakhov, V. L., Hubkin, A. A., Ponomarenko, O. L., \& Pakhomov, O. Y. (2013). Biolohichne riznomanittia Ukrainy. Dnipropetrovska oblast. Ptakhy: Horobtsepodibni (Aves: Passeriformes) [Biological diversity of Ukraine. Dnipropetrovsk region. Birds: Passerines]. Dnipropetrovsk National University Press, Dnipropetrovsk (in Ukrainian).

Chakraborty, S. K. (2021). Land-use changes: Floodplains, dams, and reservoirs integrated river basins management. Riverine Ecology, 2. Springer International Publishing, Cham.

Didukh, Y. P., Fitsailo, T. V., Korotchenko, I. A., Yakushenko, D. M., \& Pashkevych, N. A. (2011). Biotopy lisovoji ta lisostepovoji zony Ukrainy [Habitats forest and forest-steppe zone of Ukraine]. Makros, Kyiv (in Ukrainian).

Didur, O., Kulbachko, Y., Ovchynnykova, Y., Pokhylenko, A., \& Lykholat, T. (2019). Zoogenic mechanisms of ecological rehabilitation of urban soils of the park zone of megapolis: Earthworms and soil buffer capacity. Environmental Research, Engineering and Management, 75(1), 24-33.

Gaget, E., Le Viol, I., Pavón-Jordán, D., Cazalis, V., Kerbiriou, C., Jiguet, F., Popoff, N., Dami, L., Mondain-Monval, J. Y., Defos du Rau, P., Abdou, W. A. I., Bozic, L., Dakki, M., Encarnação, V. M. F., Erciyas-Yavuz, K., Etayeb, K. S., Molina, B., Petkov, N., Uzunova, D., Zenatello, M., \& Galewski, T. (2020). Assessing the effectiveness of the Ramsar Convention in preserving wintering waterbirds in the Mediterranean. Biological Conservation, 243, 108485.

Graeter, G. J., Buhlmann, K. A., Wilkinson, L. R., \& Gibbons, J. W. (Eds.). (2013). Inventory and monitoring: Recommended techniques for reptiles and amphibians. Partners in amphibian and reptile conservation technical publication IM1. Birmingham, Alabama.

Green, N. S., Wildhaber, M. L., Albers, J. L., Pettit, T. W., \& Hooper, M. J. (2020). Efficient mammal biodiversity surveys for ecological restoration monitoring. Integrated Environmental Assessment and Management, 2020, 1-13.

Hoffmann, A., Decher, J., Rovero, F., Schaer, J., Voigt, C., \& Wibbelt, G. (2010). Chapter 19. Field methods and techniques for monitoring mammals. In: Eymann, J., Degreef, J., Hauser, C., Samyn, J. Y., \& Van den Spiegel, D. (Eds.). Volume 8 - Manual on field recording techniques and protocols for all taxa biodiversity inventories. Abc Taxa Publisher. Part 2. Pp. 482-529.

Hülber, K., Moser, D., Sauberer, N., Maas, B., Staudinger, M., Grass, V., Wrbka, T., \& Willner, W. (2017). Plant species richness decreased in semi-natural grasslands in the Biosphere Reserve Wienerwald, Austria, over the past two decades, despite agri-environmental measures. Agriculture, Ecosystems and Environment, 243, 10-18

Kang, R., Shengzhi, H., Qiang, H., Hao, W., Guoyong, L., Linyin, Ch., Wei, F., \& Pei, L. (2019). A nature-based reservoir optimization model for resolving the conflict in human water demand and riverine ecosystem protection. Journal of Cleaner Production, 231, 406-418.

Karmyzova, L., \& Baranovsky, B. (2020). Flora of the Dnipro citi. Baltia Publishing, Riga.

Katanskaya, V. M. (1981). Vysshaya vodnaya rastitelnost kontinentalnykh vodoemov SSSR [Higher aquatic vegetation of the continental reservoirs of the USSR]. Nauka, Leningrad (in Russian).

Kuchynska, I. V. (2005). Ohliad deiakykh metodiv obliku vodoplavnykh ta kolovodnykh ptakhiv v umovakh rybohospodarskykh staviv [Review of some methods of counting of waterfowl and wetland birds in the conditions of fish ponds]. Branta, 8, 176-192 (in Ukrainian).

McDiarmid, R., Foster, M., Guyer, C., Gibbons, J., \& Chemoff, N. (2012). Reptile biodiversity: Standard methods for inventory and monitoring. University of $\mathrm{Ca}-$ lifornia Press, the USA.
Mosyakin, S. L., \& Fedorochuk, M. M. (1999). Vascular plants of Ukraine. Nomenclatural checklist. M. G. Kholodny Institute of Botany, Kiev.

Musakwa, W., Gumbo, T., Paradza, G., Mpofu, E., Nyathi, N. A., \& Selamolela, N. B. (2020). Partnerships and stakeholder participation in the management of national parks: Experiences of the Gonarezhou National Park in Zimbabwe. Land, 9(11), 399.

Musilová, Z., Musil, P., Zouhar, J., Adam, M., \& Bejček, V. (2018). Importance of Natura 2000 sites for wintering waterbirds: Low preference, species' distribution changes and carrying capacity of Natura 2000 could fail to protect the species. Biological Conservation, 228, 79-88.

Pavón-Jordán, D., Abdou, W., Azafzaf, H., Balaž, M., Bino, T., Borg, J. J., Božǐč, L., Butchart, S. H. M., Clausen, P., Sniauksta, L., Dakki, M., Devos, K., Domsa, C., Encarnaçao, V., Etayeb, K., Faragó, S., Fox, A. D., Frost, T., Gaudard, C., Georgiev, V., Goratze, I., Hornman, M., Keller, V., Kostiushyn, V., Langendoen, T., Ławicki, Ł., Ieronymidou, C., Lewis, L. J., Lorentsen, S.-H., Luigujoe, L., Meissner, W., Mikuska, T., Molina, B., Musil, P., Musilova, Z., Nagy, S., Natykanets, V., Nilsson, L., Paquet, J.-Y., Portolou, D., Ridzon, J., Santangeli, A., Sayoud, S., Śćiban, M., Stipniece, A., Teufelbauer, N., Topić, G., Uzunova, D., Vizi, A., Wahl, J., Yavuz, K. E., Zenatello, M., \& Lehikoinen, A. (2020). Positive impacts of important bird and biodiversity areas on wintering waterbirds under changing temperatures throughout Europe and North Africa. Biological Conservation, 246, 108549.

Perrings, C., Naeem, S., Ahrestani, F., Bunker, D. E., Burkill, P., Canziani, G., Elmqvist, T., Ferrati, R., Fuhrman, J., Jaksic, F., Kawabata, Z., Kinzig, A., Mace, G. M., Milano, F., Mooney, H., Prieur-richard, A.-H., Tschirhart, J., \& Weisser, W. (2010). Ecosystem Services for 2020. Science, 330(6002), 323-324.

Pittock, J., Finlayson, C. M., \& Arthington, A. H. (Eds.). (2017). Freshwater ecosystems in protected areas: Conservation and management. Routledge, London.

Ponomarenko, O. L. (2005). Vplyv transformatsiinykh protsesiv u lisovykh ekosystemakh na zhyttiediialnist ptakhiv [Influence of transformation processes in forest ecosystems on bird life]. Dnipropetrovsk University Press, Dnipropetrovsk (in Ukrainian).

Richardson, D. M., Pyšek, P., Rejmánek, M., Barbour, M. G., Panetta, F. D., \& West, C. J. (2000). Naturalization and invasion of alien plants: Concepts and definitions. Diversity and Distributions, 6, 93-107.

Schindler, S., O’Neill, F., Biró, M., Damm, C., Gasso, V., Kanka, R., Sluis, T., Krug, A., Lauwaars, S. G., Sebesvari, Z., Pusch, M., Baranovski, B., Ehlert, T., Neukirchen, B., Martin, J. R., Euller, K., Mauerhofer, V., \& Wrbka, T. (2016). Multifunctional floodplain management and biodiversity effects: A knowledge synthesis for six European countries. Biodivers Conserve, 25, 1349-1382.

Sigwart, J. D., Bennett, K. D., Edie, S. M., Mander, L., Okamura, B., Padian, K. Wheeler, Q., Winston, J. E., \& Yeung, N. W. (2018). Measuring biodiversity and extinction-present and past. Integrative and Comparative Biology, 58(6), 1111-1117.

Tarasov, V. V. (2012). Flora Dnipropetrovskoji ta Zaporizkoji oblastej [Flora of Dnipropetrovsk and Zaporizhia regions]. Lira, Dnepropetrovsk (in Ukrainian).

Thieme, M. L., Khrystenko, D., Qin, S., Golden Kroner, R. E., Lehner, B., Pack, S., Tockner, K., Zarfl, C., Shahbol, N., \& Mascia, M. B. (2020). Dams and protectted areas: Quantifying the spatial and temporal extent of global dam construction within protected areas. Conservation Letters, 13(e), 12719.

Tomialojc, L. (1976). Skroty i znaki zalecane w badaniach ilosciowych nad ptakami [Abbreviations and characters recommended for bird quantification]. Notatki Ornitologiczne, 17, 40-44 (in Polish).

Tomialojc, L. (1980). Kombinowana odmiana metody kartograficznej do liczenia ptakow legowych [A combined variation of the cartographic method for counting breeding birds ]. Notatki Ornitologiczne, 21, 33-54 (in Polish).

Torre, I., Raspall, A., Arrizabalaga, A., \& Díaz, M. (2018). SEMICE: An unbiased and powerful monitoring protocol for small mammals in the Mediterranean Region. Mammalian Biology, 88, 161-167.

Yermolenko, S. V., Hahut, A. M., \& Gasso, V. Y. (2016). Izmenchivost morfometricheskih priznakov vodianogo uzha Natrix tessellata (Reptilia, Colubridae) Tsentral'nogo i Yuzhnogo Pridneprov'ia [Variation in morphological characters of the dice snake Natrix tessellata (Reptilia, Colubridae) in the Central and Southern Dnieper River Area]. Visnyk of Dnipropetrovsk University, Biology, Ecology, 24(2), 526-530 (in Russian). 